University of Tennessee Health Science Center

UTHSC Digital Commons

\title{
Root Morphology and Sexual Dimorphism : Size Standards for Crown and Root Dimensions in Adolescents
}

\author{
William Max Couch \\ University of Tennessee Health Science Center
}

Follow this and additional works at: https://dc.uthsc.edu/dissertations

Part of the Orthodontics and Orthodontology Commons

\section{Recommended Citation}

Couch, William Max, "Root Morphology and Sexual Dimorphism : Size Standards for Crown and Root Dimensions in Adolescents" (2007). Theses and Dissertations (ETD). Paper 48. http://dx.doi.org/ 10.21007/etd.cghs.2007.0058. 


\title{
Root Morphology and Sexual Dimorphism : Size Standards for Crown and Root Dimensions in Adolescents
}

\author{
Abstract \\ "Early" or two-phase orthodontic treatment of Class II malocclusions is a highly debated topic in the \\ orthodontic literature. We report here on a retrospective cephalometric study of patients with Class II, \\ division 1 malocclusions. One group consisted of 32 consecutively treated patients who received "early" \\ treatment with a Fränkel II appliance followed by treatment with full fixed appliance. These subjects were \\ "matched" by sex and cephalometric value, to a subject treated with standard edgewise appliances in a \\ single phase. Matching criteria focused on the bony facial characteristics, notably ANB, NAP, Y Axis, \\ AOBO, FMA, overjet, and overbite. The question was whether the cephalometric results at the end of \\ treatment were comparable. Two-phase subjects were treated on average 2 years longer than the one- \\ phase edgewise group. Neither integumental variable ( $Z$ angle, E plane) differed statistically. Two of eight \\ skeletal variables differed statistically, namely ANB and FMA. ANB was on average $1.3^{\circ}$ smaller in the \\ two-phase group, while the FMA was on average $1.8^{\circ}$ steeper in the one-phase group. Four of the eleven \\ dental variables differed statistically between the two groups. These variables were related to incisor \\ position. Overall, the incisors in both treatment groups proclined during treatment. The two-phase \\ subjects had a lower rate of premolar extraction (3\% vs. $56 \%$ ) than the one-phase subjects. Similar \\ skeletodental endpoints were achieved regardless of treatment protocol; indicating the treatments can be \\ viewed as equivalent approaches to a common problem.

\section{Document Type} \\ Thesis

\section{Degree Name} \\ Master of Dental Science (MDS)

\section{Program} \\ Dental Science (Orthodontics)

\section{Research Advisor} \\ Edward F. Harris, PhD

\section{Keywords} \\ Tooth dimensions, Odontometrics, Incisors, Tooth crown, Sexual demorphism

\section{Subject Categories} \\ Dentistry | Medicine and Health Sciences | Orthodontics and Orthodontology
}




\title{
ROOT MORPHOLOGY AND SEXUAL DIMORPHISM: SIZE STANDARDS FOR CROWN AND ROOT DIMENSIONS IN ADOLESCENTS
}

\author{
A Thesis \\ Presented for \\ The Graduate Studies Council \\ The University of Tennessee \\ Health Science Center
}

\author{
In Partial Fulfillment \\ Of the Requirements for the Degree \\ Master of Dental Science \\ From The University of Tennessee
}

By

William Max Couch, Jr., D.D.S.

May 2007 
Copyright ( 2007 by William Max Couch, Jr.

All rights reserved 


\section{DEDICATION}

This thesis is dedicated to my parents, Dr. William M. Couch and Mrs. Sara H. Couch, and my wife, Holly R. Couch, in recognition of their love, guidance and support of my return to pursue a career in orthodontics and further my education. 


\section{ACKNOWLEDGEMENTS}

I would like to thank my thesis committee for their input and direction during my enrollment in the postdoctoral graduate program. Dr. Edward Harris, my committee chairman and research advisor, guided and instructed me

during the entire research project. Dr. Chuck Robinson and Dr. Marjorie Woods attended my meetings, making comments and suggestion for improvement. I also deeply appreciate the support of my wife, Holly, as I persevered on this project and increased her own responsibilities in the process. Thank you for being a trooper. Finally, I would like to thank my son, Liam, for putting up with my long hours at work and weekend time spent on this project. 


\begin{abstract}
Incisors are the teeth that experience the most crowding, rotation and displacement in the typical malocclusion. They also are the most visible teeth, thus, the focus of most lay peoples' perception of occlusion and dental esthetics. In addition, incisors often are moved appreciably during orthodontic treatment, and they characteristically experience more root resorption than other tooth types. These several considerations led to the current study that establishes baseline data on a cohort of adolescent American whites. OBJECTIVE: By establishing contemporary metric crown and root dimensions in a sample at pretreatment, we may better understand the normative incisor crown-root size and shape relationships. METHODS: Pretreatment periapical radiographs and casts were collected for a total of 148 adolescents, 51 males and 97 females, examined between the ages of 9 to 19 years of age. Mesiodistal crown widths for all eight incisors were measured from dental casts with sliding calipers, and the same patients' periapical radiographs were scanned on a flatbed scanner. Commercial software was used to obtain the desired measurements. Five variables were measured: mesiodistal crown width, crown height, root length, crown/root ratio, and crestal bone height. RESULTS: Of the four incisor tooth types, mesiodistal crown width of just the maxillary central incisor (U1) exhibited significant sexual dimorphism (at about 4\%). For crown height, just
\end{abstract}


the mean size difference for the maxillary lateral incisor (U2) was significantly dimorphic between the sexes (a $4 \%$ difference), and crown heights of the mandibular incisors were virtually identical in the two sexes. The dimorphism in incisor root lengths was in the range of 5 to $8 \%$, which is noticeably higher than for crown dimensions. Crown-root ratios exhibited significant dimorphism for the mandibular incisors only, mainly due to the longer roots in males than females. Only the maxillary lateral incisor (U2) yielded substantive evidence of size difference between Angle's Classes, with Class I cases (controlled for sex) having smaller root dimensions than the Class II cases. CONCLUSIONS: Distal root lengths and bone heights were systematically shorter than the corresponding mesial root lengths and bone heights for all of four tooth types. Alveolar crestal bone heights were about a millimeter apical to the tooth's cementoenamel junction. Crown-root ratios for all tooth types were on the order of $50 \%$, showing that root length was about twice the crown length. For crown width ratios, even though males have larger teeth than females, they still have the same crown proportionalities between tooth types. This study of incisor crown-root dimensions in a contemporary American white sample indicates that root lengths are somewhat more sexually dimorphic than crown dimensions and, thus, are a bit more useful for sex determination. The main focus of the study was to provide normative statistics for crown and root dimensions, by sex, tooth type, and Angle's molar classification. 


\section{TABLE OF CONTENTS}

CHAPTER

PAGE

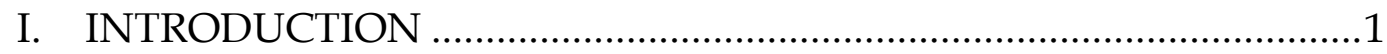

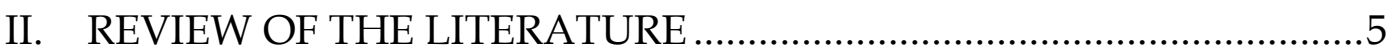

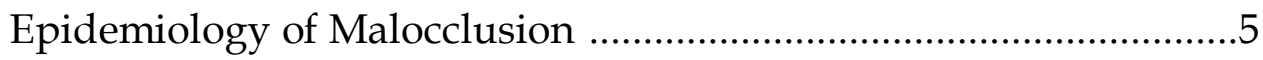

Class II, Division 2 Incisor Morphology …………….........................6

Crown Size to Root Length Correlation................................................8

Anterior Tooth Size Discrepancies ....................................................8

Tooth Anomalies Related to Malocclusion........................................11

Sex Differences in Tooth Size...........................................................13

Race Differences in Tooth Size..........................................................15

Alveolar Bone Heights ....................................................................17

Bilateral Asymmetry in the Permanent Teeth....................................20

Tooth Formation and Eruption Patterns.............................................20

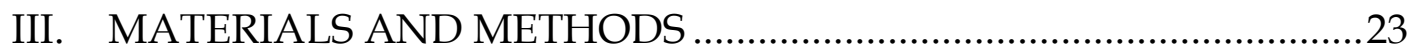

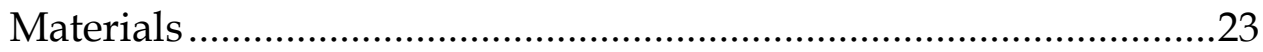

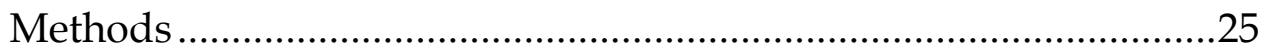

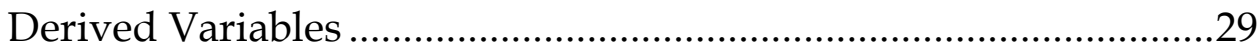

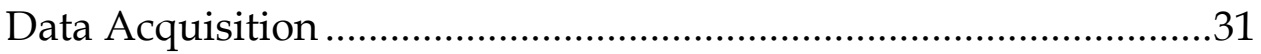

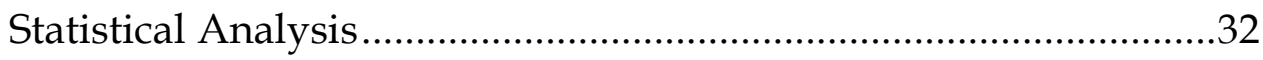

vii 
Research Goals .

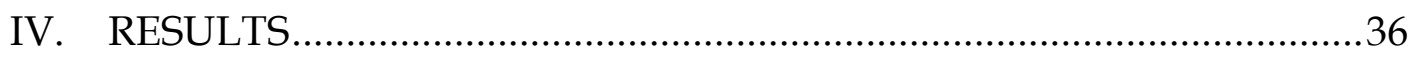

Evaluation of Normality …………....................................................36

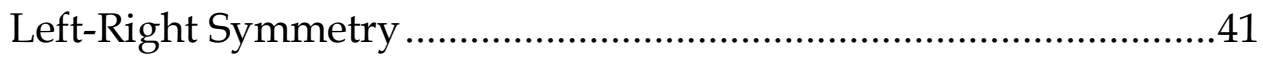

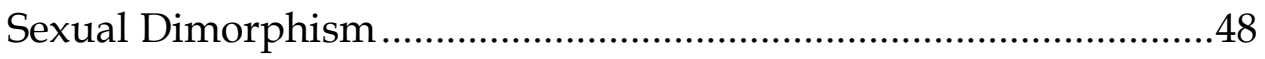

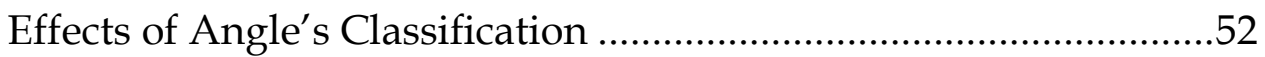

Principal Components Analysis .......................................................56

Crown Heights and Root Lengths......................................................74

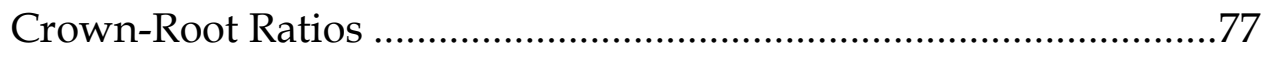

Crown and Root Lengths ................................................................

Crown Height-Width Ratios.............................................................. 80

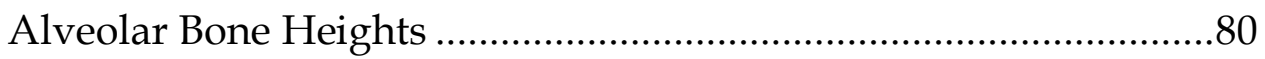

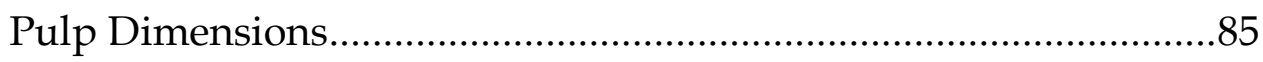

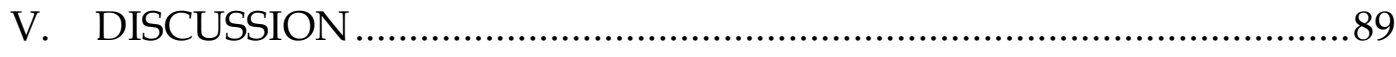

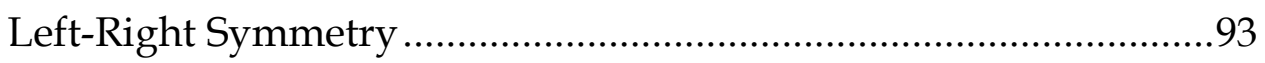

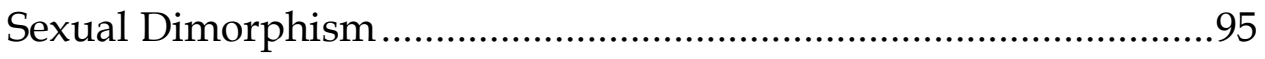

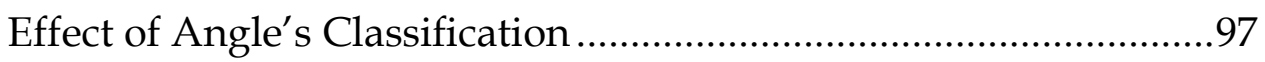

Crown Heights and Root Lengths....................................................99

Mesiodistal Crown Width Ratios ..................................................102

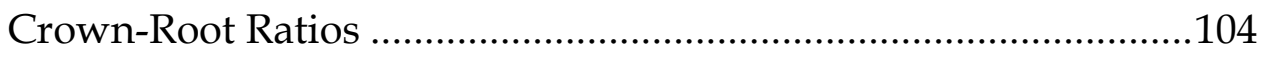

Alveolar Bone Heights ....................................................................105

viii 
VI. SUMMARY AND CONCLUSIONS...........................................107

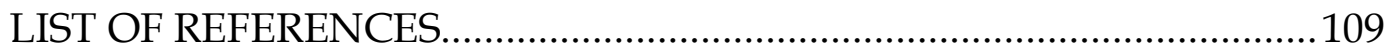

APPENDIX. HISTOGRAMS OF MEAN TRAIT SIZES BY

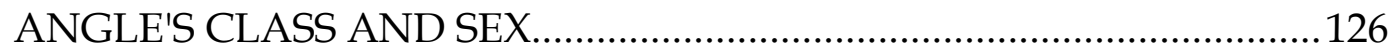

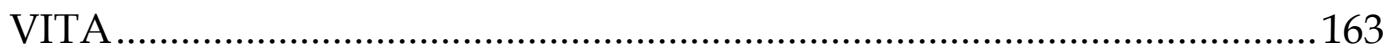




\section{LIST OF TABLES}

TABLE

PAGE

1. Tests for normal distributions of bone and root dimensions in males.

2. Tests for normal distributions of bone and root dimensions in females.

3. Tests for normal distributions in tooth crown diameters in males

4. Tests for normal distributions in tooth crown diameters in females

5. Paired $t$ tests comparing left and right dimensions of root lengths and crestal bone heights.

6. Paired $\mathrm{t}$ tests comparing side differences in mesiodistal

crown dimensions

7. ANOVA tests for sexual dimorphism in crown, root, and crestal bone dimensions

8. Tests for sexual dimorphism in mesiodistal crown dimensions.

9. Results of two-way analysis of variance, testing for size differences by Angle's classification while controlling for patient's sex

10. Results of two-way analysis of variance for mesiodistal crown widths, testing for size differences by Angle's classification while controlling for patient's sex..

11. Pairwise correlations for the maxillary central incisor (sexes combined) 
12. Results of principal components analysis for seven variables from the maxillary central incisor, derived from the covariance matrix

13. Pairwise correlations for the maxillary lateral incisor (sexes combined)

14. Results of principal components analysis for seven variables from the maxillary lateral incisor, derived from the covariance matrix.

15. Results of two-way analysis of variance, testing for differences by class or sex in principal components scores for the maxillary central incisor.

16. Results of two-way analysis of variance, testing for differences by class or sex in principal components scores for the maxillary lateral incisor.

17. Descriptive statistics and tests for sexual dimorphism of root and crown heights.

18. Descriptive statistics and test for sexual dimorphism of crown-root ratios.

19. Ratios of lateral to central incisor widths, by sex, in the two arcades.

20. Descriptive statistics of alveolar bone heights and tests for sexual dimorphism

21. Paired comparisons of bone heights between sides of each tooth, medial and lateral

22. Sex-specific descriptive statistics for pulp height and distance from pulp to incisal edge, along with ANOVA tests for a difference between the means. 


\section{LIST OF FIGURES}

FIGURE

PAGE

1. Diagrammatic faciolingual radiographic view of a maxillary central incisor, showing the points digitized on each of the four incisor tooth types

2. Diagrammatic faciolingual radiographic view of a maxillary lateral incisor

3. Diagrammatic faciolingual radiographic view of mandibular central and lateral incisors. .28

4. Mean scores for the maxillary central incisor, PC One ............................65

5. Mean scores for the maxillary central incisor, PC Two.............................66

6. Mean scores for the maxillary central incisor, PC Three .........................67

7. Mean scores for the maxillary central incisor, PC Four ...........................68

8. Mean scores for the maxillary lateral incisor, PC One ...........................70

9. Mean scores for the maxillary lateral incisor, PC Two ..........................71

10. Mean scores for the maxillary lateral incisor, PC Three .........................72

11. Mean scores for the maxillary lateral incisor, PC Four ...........................73

12. Comparisons of mesiodistal crown widths between G. V. Black's data and the present study (where data are presented by sex).

13. Comparisons of overall tooth lengths between G. V. Black's data and the present study (where data are presented by sex). 


\section{CHAPTER I}

\section{INTRODUCTION}

There is considerable evidence that orthodontic tooth movement can cause external apical root resorption (Harris 2000). In contrast, there seems to be no systematic root resorption in people as they age who have not had orthodontic treatment (Baker and Harris 1990; Woods et al. 1990; Bishara et al. 1999). In the present study our intent is to better understand root morphology in patients to prevent unnecessary iatrogenic harm when treated orthodontically. By establishing contemporary metric crown and root standards in a sample of cases at pretreatment, we may better understand the nature, causes, and severity of external apical root resorption.

There have been numerous studies of crown and root morphology. G. V. Black (1897) published detailed measurements of the teeth, and these have been broadly referenced for over a century. Black reported on crown length, root length, mesiodistal and buccolingual crown diameters, and curvature of the gingival line. These were direct measurements of extracted human teeth, though of unknown origin (reviewed in Harris and Burris 2003).

Kramer and Ireland (1959) investigated metric and morphological details of the primary teeth. They made some 45 measurements of each posterior tooth type, and more than 1,600 teeth were studied. Their anterior 
tooth values correspond to those published by Black, though they noted that it was difficult to get sound specimens of the anterior teeth due to root resorption caused by the permanent tooth successors. On the other hand, posterior tooth dimensions differed from those described by Black, which Kramer and Ireland attributed to their more precise methods of measurement.

Harris and Burris (2003) reported on permanent tooth dimensions from a contemporary sample of American Whites and made comparisons to G. V. Black's data collected in the 1800s. Harris and Burris made sliding caliper measurements on dental casts versus Black who used extracted teeth. Parenthetically, sliding calipers had not been invented when Black collected his measurements. Black probably used draftman's dividers, reading the millimetric sizes by holding the divider's beaks against a ruler (Harris, pers. comm.). Harris and Burris' study generated data statistically different from Black's. Some contributing factors to the differences are that his sample sizes may have been small; racial composition of his sample may have differed; he may have measured the teeth using different methods; and it can only be assumed that he would not have measured teeth with obvious interproximal wear and/or occlusal attrition. It is also contemplated that a secular trend over the past century could be invoked, so that some of the crown dimensions have become smaller and others larger across generations. But, secular trends would seem to be an unlikely cause, at least for the bulk of comparisons. Comparing 
the Harris-Burris data to Black's, there are a number of dimensions that enlarged through time and others that diminished - there was not any systematic trend. Because of the strong, positive intercorrelations among crown diameters (e.g., Moorrees and Reed 1964), one would expect secular trends to be in the same direction, particularly within morphogenetic fields. The secular trends that have been documented for tooth size (e.g., Garn et al. 1968; Ebeling et al. 1973; Harris et al. 2001) show that all tooth dimensions change in the same direction in proportionate fashions.

Tooth dimensions of males are generally larger than their female counterpart (e.g., Garn et al. 1967, 1978). In primary and permanent dentitions, the upper canines and central incisors show the greatest percentage sex differences, whereas the upper laterals and lower centrals are the most similar between the sexes (Doris et al. 1981).

Research has also disclosed differences in root morphology based on Angle's molar classification. The maxillary central incisor varies in its morphology in Class II, division 2 and Class III patients (Delivanis and Kuftinec 1980; Harris et al. 1993). Cephalometric radiographs were measured from patients with different malocclusions, and a significantly greater crownto-root angle was noticed in maxillary central incisors viewed from the lateral aspect in Class II, division 2 and Class III subjects. This angle referred to as the "collum angle" is formed by the junction of the long axis of the root and that of 
the crown.

The purpose of the present study is to provide size standards for incisor crown and root dimensions in adolescents. The study generated normative data, corrected for magnification, on healthy adolescents slated for full-banded appliance orthodontic treatment. The incisors, which are the tooth types most susceptible to external apical root resorption (Harris, 2000) were analyzed. Variables measured consist of crown, root, and tooth length; crown to root ratio, crestal bone height, and pulp length.

Data were collected from periapical films using a computer-assisted photogrammetric method. Mesiodistal tooth crown dimensions measured from the individual's dental casts were used to correct for radiographic magnification.

This is a descriptive study of adolescents who subsequently were treated orthodontically, but only the pretreatment records are studied here. Consequently, the data are drawn from a sample of convenience, though we made an effort to collect representative samples of patients with Class I, Class II, and Class III malocclusions (Angle). This study is restricted to American Whites of Western European extraction living in the Midsouth because of known ethnic differences in tooth and root dimensions (e.g., Kieser 1990). 


\section{CHAPTER II}

\section{REVIEW OF THE LITERATURE}

\section{Epidemiology of Malocclusion}

By the 1970s, a series of studies by universities and public health groups in most developed countries provided a reasonably clear worldwide picture of the prevalence of various occlusal relationships of the dentition. From 1989 to 1994, estimates of malocclusion were obtained in the United States as part of a large scale national survey of health care problems. This is labeled the National Health and Nutrition Estimates Survey III (Kelly and Harvey 1977; NHANES III; Proffit 2000). A measure of incisor alignment, the prevalence of a midline diastema greater than $2 \mathrm{~mm}$, and the prevalence of a posterior crossbite were some of the characteristics of malocclusion evaluated. Also, overjet and overbite were measured. The survey noted that just over half of the children age 8 to 11 had well aligned incisors. That percentage decreased by ages of 12 to 17 as the remaining permanent teeth erupted. The survey found that occlusal relationships in the upper arch remain roughly stable through adulthood, but the lower arch worsens over time. Severe crowding of the incisors was reported in $15 \%$ of adolescents and adults, such that extractions would be needed to correct their alignment. The survey found American Blacks were more than twice as likely to have a midline diastema as 
American Whites. In addition, $26 \%$ of children had a midline diastema that normally closes with the subsequent eruption of other permanent teeth. Severe deepbite, which was defined as in excess of $5 \mathrm{~mm}$, affected about $20 \%$ of children and $13 \%$ of adults. Openbites occurred in less than $1 \%$ of the population. The severe deepbites occurred almost twice as often in Whites as Blacks, while anterior openbites were about five times more prevalent in Blacks (Proffit 2000).

\section{Class II, Division 2 Incisor Morphology}

The crown-root shape of the maxillary central incisor in the Class II, division 2 patient differs from that in the other Angle categories of malocclusion. Incisor shape characteristics involved axial torsion (i.e., a reduced collum angle), reduced labiopalatal root thickness, shorter root length, and greater crown height (McIntyre and Millett 2003). Class II, division 2 patients have a statistically significant increase in the deviation of the collum angle from the other malocclusion groups when measuring cephalometric radiographs (Delivanis and Kuftinec 1980; Harris et al. 1993).

Lapatki et al. (2002) evaluated the relationship between resting lip pressure and the level of the lip line on the maxillary central incisor to the causes of Class II, division 2 malocclusion. Their study showed that individuals with this malocclusion have upper central incisors exposed to 
significantly higher lip pressures than those with Class I malocclusions. This was primarily attributed to a high lip line and not to hypertonic perioral musculature. The results of this study support the inference that local genetic factors play an important role in the cause of upper central incisor retroinclination of Class II, division 2 individuals. The vertical relation between the lips and the maxillary dentoalveolar structure is unbalanced, resulting in excessive resting lip pressure, causing lingual tipping of the upper incisor crowns.

Bryant et al. (1984) showed that the permanent maxillary central incisor variously exhibits three morphological features related to the type of malocclusion. The central incisor crowns of Class II, division 2 patients were found to be "bent" lingually. The orthodontist should take care treating these patients not to impinge on the palatal cortical bone, because it could promote root resorption (Kaley and Phillips 1991). Also, the labial surface angle of the maxillary central incisors was found to vary widely, though not systematically between Angle classes of malocclusion. This variation can cause problems finishing orthodontic cases when using the same pre-torqued and preangulated brackets on every case. Also, the lingual surface curvature was found to vary widely in shape, but none was particular to a certain Angle malocclusion. 


\section{Crown Size to Root Length Correlation}

Adjacent teeth exhibit the highest root length correlations (Garn, Van Alstine and Cole 1978). Garn et al. also found that root length and crown size were systematically and positively correlated for both mesiodistal and buccolingual diameters. They further determined that mesiodistal crown size showed higher correlations with root lengths than did buccolingual crown size.

Townsend et al. (1978) and Kolakowski et al. (1981) reported that the size and shape of the crowns of the teeth appear to be under moderately strong genetic control. In contrast, root morphology seems to be fairly susceptible to local environmental factors. Others reported that root dimensions are not strongly correlated with crown size or jaw size, and their morphology can be highly variable (e.g., Anderson et al. 1977; Garn et al. 1980).

Garn et al. $(1962,1963,1964)$ reported that the root mean square size is greater for the more distal tooth in each morphological class (e.g., lateral incisor, second premolar, and third molar). These studies were in accordance with their previous findings on increased size variation and lower size likenesses for the more distal teeth of each class.

\section{$\underline{\text { Anterior Tooth Size Discrepancies }}$}

Araujo and Souki (2003) studied Bolton anterior tooth size discrepancies among different malocclusion groups. They reported a total of $56 \%$ of the 
subjects in their study had a Bolton tooth size discrepancy (greater than $1 \mathrm{sd}$ of the idealized mean). Also, clinically significant discrepancies were found in $23 \%$ of their sample. They concluded that individuals with Angle Class I and Class III malocclusions have significantly greater frequencies of tooth size discrepancies than individuals with Class II malocclusions. They also reported that the mean anterior tooth size discrepancy for Angle Class III subjects was much greater than for Class I and Class II subjects. In contrast, no significant difference in Bolton anterior ratios was found as a function of sex.

Gilpatric (1923) studied 2,000 individuals, finding that the upper teeth should be 8 to $12 \mathrm{~mm}$ larger than the corresponding lower teeth. He measured the combined mesiodistal widths of each arch between and including first molars. If the difference between the arches was over 8 to $12 \mathrm{~mm}$, the result would be excess overbite (Gilpatric 1923; Stanton 1928). This early study stressed the importance of recognizing tooth size discrepancies. In order to achieve optimum occlusion during orthodontic treatment, these discrepancies need to be accounted for during treatment.

Bolton $(1959,1962)$ established that an ideal anterior ratio had a mean value of 77.2 with a standard deviation of 1.65. This value is defined as the ratio of the combined mesiodistal crown diameters of the mandibular anterior teeth (incisors and canines) divided by the combined mesiodistal crowns of their maxillary counterparts. Bolton noted that it would be difficult to obtain 
an excellent occlusion in the finishing phases of treatment without an appropriate mesiodistal tooth size ratio. Orthodontists should be concerned with tooth size discrepancies because of their high prevalence among orthodontic patients. Bolton reported that $29 \%$ of the patents studied in his private practice had a discrepancy in excess of one standard deviation. Richardson and Malhotra (1975) reported a similar discrepancy - 34\% - of their American Black patients.

Lavelle (1972) wrote that Bolton discrepancies are more common in Class III individuals due to all the teeth in the lower arch being larger statistically than those in Class I and Class II individuals. Smith et al. (2000) reported that there was no statistical difference in Bolton ratios between males and females.

Laino et al. (2003) conducted a study to explore if there was a significant correlation between the posterior, the anterior, and the total Bolton analyses. Secondarily, their study investigated the prevalence of tooth size discrepancies as related to skeletal malocclusion in a Campanian sample. They reported that there was no evidence of any predisposition for a tooth size discrepancy in any malocclusion group. They postulated that other authors reached their conclusions through comparisons of mean values in the three malocclusion groups. Laino and coworkers disagreed with previous studies because they thought it was impossible to extrapolate absolute values, such as the 
mesiodistal tooth size, from the Bolton analysis. They also reported that differences in the ethnicity of patient populations must be taken into account, and they concluded that tooth size discrepancy cannot be the sole determining factor of skeletal malocclusion.

\section{Tooth Anomalies Related to Malocclusion}

Cua-Benward and coworkers (1992) studied the prevalence of missing teeth in different malocclusion groups, relating their findings to Moss' functional matrix model. They found a greater prevalence of tooth deformities in the maxillae of Class III individuals and more tooth size deformities in the mandible of Class II individuals.

Basdra et al. (2001) examined cases with Class II division 1 and Class III malocclusions for the existence of congenital tooth anomalies, such as tooth agenesis, impacted canines, peg-shaped laterals, transpositions, and supernumerary teeth. The rationale was that Class II division 2 has been related to small teeth by Peck and coworkers (1998), and the Angle classification has been suggested to be a highly heritable craniofacial type (Markovic 1992; Mossey 1999). Basdra and coworkers observed upper lateral incisor agenesis in $6 \%$ of the Class III subjects and in $2 \%$ of the Class II division 1 subjects; these percentages are similar to the general population frequencies of 0.5 to 3\% (Schulze 1982; Bredy et al. 1991). Peg-shaped upper lateral incisors 
were observed in $3 \%$ of the Class III patients and in about $1 \%$ of the Class II division 1 patients. It was also observed that upper canines were impacted in $9 \%$ of their Class III sample and in about $1 \%$ of the Class II division 1 patients. Previously, general population studies reported that impacted canines occurred in between 1 and 3\% when combining all Angle malocclusion categories (Dachi and Howell 1961; Thilander and Jakobsson 1968).

Transpositions were only found in Class III patients but rarely (at only $0.5 \%$ ). Supernumerary teeth were found in about $1 \%$ of Class II division 1 patients and in about $4 \%$ of Class III patients. This was consistent with frequencies found in the general population (Luten 1967), and all were found in the maxillary arch, of which half were mesiodens.

Overall, both Class III and Class II division 1 patients exhibited frequencies of congenital anomalies that are fairly similar to those of the general population. There was no statistically significant difference in tooth anomalies between Class III and Class II division 1 patients, but there were differences when compared with frequencies in Class II division 2 subjects (except when comparing supernumerary teeth):

It seems that, apart from craniofacial characteristics, other factors such as congenital tooth anomalies are related to malocclusions. The present research together with previous work (Peck et al. 1998; Baccetti 1998; Basdra et al. 2000) provides evidence for the existence of a specific relationship of certain congenital tooth anomalies with specific malocclusions [Basdra et al. 2001:150] 
Basdra and coworkers (2000) suggested that these abnormalities are most likely associated with defects in genes associated with tooth development. In Class II division 2 subjects, they reported more females having tooth anomalies than males. Agenesis of a maxillary lateral incisor was most frequently reported bilaterally, and bilateral peg-shaped laterals were seen more commonly in females. In agreement with Peck et al. (1998) and in summarizing the results of this study, Basdra et al. concluded that the Class II division 2 malocclusion is closely related to congenital tooth anomalies.

Peck and coworkers (1998) studied incisor crown size related to Angle Class II division 2 malocclusion. They reported that the average maxillary incisor mesiodistal crown widths were smaller in the Class II division 2 sample than in the control sample. Peck suggested that significant reductions in the mesiodistal incisor widths indicate a pattern of smaller-than-average teeth as a characteristic of this malocclusion group.

\section{$\underline{\text { Sex Differences in Tooth Size }}$}

Garn et al. (1967) studied sexual dimorphism of teeth in the buccolingual dimension whereas most previous studies described the mesiodistal tooth dimension. They reported that sexual dimorphism averaged $5.6 \%$ for the buccolingual tooth diameter as compared to $4.2 \%$ for the mesiodistal diameter of the same tooth. Garn et al. also noted that there are discernible sex 
differences in tooth shape, with males tending toward a more nearly square shape and females showing greater size diminutions buccolingually than mesiodistally.

Males have larger tooth dimensions on average than females (e.g., Garn et al. 1967; Townsend 1979; Harris and Bailit 1987) and statistically significant sexual dimorphism encompasses both the primary and permanent dentitions. Moss et al. (1977) tried to account for this difference in size by conjecturing that, since males tend to spend more time than females undergoing amelogenesis (at least according to one study, Moorees et al. 1963), sexual dimorphism in crown size could be due to males' thicker enamel. But this conjecture was disproved with the benefit of direct studies of human tooth enamel thickness that reported no significant marginal enamel thickness between the sexes (e.g., Alvesalo and Tammisalo 1981; Stroud et al. 1994, 1998; Harris and Hicks 1998). Harris and Hicks (1998) studied periapical radiographs of the four permanent maxillary incisors and reported that the sexual dimorphism was related to dentine differences between the sexes instead of enamel thicknesses. This sexual dimorphism averaged about $6.5 \%$ in the American whites used in the study, and seems to be due the size of the inner enamel epithelium attained at the end of the bell stage of tooth formation.

Harris et al. (2001) studied the tissue contributions to sex and race related to differences in tooth crown size of primary molars. This study 
assessed size of constituent primary tooth crown components (enamel, dentine, and pulp) to understand the manner in which males characteristically have larger teeth than females. They found that, overall, males have dentine thicknesses that are $4 \%$ greater than in females. There was no difference in enamel thickness or pulp chamber width between males and females. So, the mesiodistal crown diameter is significantly larger in males than females, averaging $3 \%$, and this is primarily attributable to the dentine component of the deciduous tooth.

Woods et al. (1990) studied the age-progressive changes in pulp widths and root lengths during adulthood in American Blacks and Whites. Roots were appreciably longer in Blacks of all ages. Age had a marked effect on pulp widths in all three tooth types in the study (maxillary central incisor, mandibular canine, and mandibular second molar). It was reported that incisor pulp width shows neither a race nor sex difference. It was also noted that males have longer roots than females; also, for the incisor, sex is a more important determinant of root length than race. This root length dimorphism is $4 \%$ for the maxillary central incisor.

\section{$\underline{\text { Race Differences in Tooth Size }}$}

Woods and coworkers (1990) reported that the roots are appreciably longer in American Blacks than Whites. The maxillary central incisor was 3\% 
longer in Blacks. Blacks are known to have larger tooth crown diameters than Whites (e.g., Richardson and Malhotra 1975; Macko et al. 1979), and Woods' study reported that these differences extended to some pulp chamber dimensions and to measures of root size. It was noted that, in contrast, the maxillary central incisor pulp chamber width showed no race difference.

Harris et al. (2001) studied the tissue contributions in the primary molars, and they reported that American Blacks have statistically significantly larger crown diameters than American Whites. This has been shown to be true for both the primary and permanent dentitions (e.g., Richardson and Malhotra 1976; Farmer 1990; Harris and Rathbun 1989, 1991; Vaughan and Harris 1992).

Harris and coworkers (2001) confirmed that the mesiodistal crown diameters were significantly larger in Blacks than Whites. Most of which was attributed to the greater marginal enamel thicknesses in Blacks than in Whites. The thickness of the dentine was also a contributing factor, but the actual pulp diameter was found to be greater in Whites than Blacks. It is also noteworthy that American Blacks spend less time in all phases of crown and root formation, even though their teeth are larger, which suggests that mitotic rates also differ substantially between these races (Harris and McKee 1990, 1995; Mincer et al. 1993). 


\section{$\underline{\text { Alveolar Bone Heights }}$}

Alveolar marginal bone height decreases with increasing age and with deterioration of oral hygiene (e.g., Schei et al. 1959; Lavstedt et al. 1975;

Markkanen et al. 1981). Bergström et al. (1986) reported alveolar bone height as a percentage of the root length. They focused on musicians of wind blown instruments compared to a control group of musicians of other instruments. Root measurement was the mean of the distance from the mesial and distal cementoenamel junction (CEJ) to the apex of the root of the tooth. Alveolar bone height was the mean distance from the mesial and distal crestal bone to the root apex. It was concluded that in the absence of pathology and presence of good oral hygiene, there was no significant difference between the two groups of musicians. Additionally, they found no difference between men and women for this variable. However, with observations of alveolar bone height as given in relation to root length, teeth with long roots will be favored as compared to those with shorter ones. To what extent the alveolar bone height values observed are influenced by the method of determination is not fully known and remains to be evaluated.

Salonen et al. (1991) reported that women had a significantly higher mean bone height to root length ratio $(\mathrm{B} / \mathrm{R})$ compared to men when adjusted for age. Women do have shorter roots than men, but this did not seem to influence their findings as previously suggested by Bergström et al. (1986). 
Also, a reduced alveolar bone level at the older ages was noticed compared with the younger age groups. Wouters et al. (1993) reported that current smokers exhibited significantly lower B/R ratio values than non-smokers. But no statistically significant relationship between smokeless tobacco and B/R ratio was found. Their findings are based on a radiographic cross-sectional epidemiologic study of an adult Swedish population.

Polson et al. (1981) and Ericsson et al. (1982) concluded that other factors besides age and oral hygiene influence alveolar bone height. Excessive forces exerted on teeth and on the periodontium, like trauma from occlusion, may increase loss of alveolar bone height in the presence of inflammation.

Root area measurements have been quantified in various studies. Previous measurement methods were cumbersome or inaccurate, required tedious laboratory procedures, or did not measure true surface area. Chen and coworkers (2004) used a novel technique for looking at root surface area. They measured extracted premolars by digitizing the root surfaces in all three dimensions. The data were processed using engineering application software, and length, projection area, and surface area of the root were computed. From these three measurements, the accuracy of the supported surface area ratio calculations at the different lengths of the root were evaluated. They found that linear measurements overestimated root coverage, and that their method was more accurate in determining true root surface area. 
Lamedin et al. (1992) presented a method for age determination of adults from single-rooted teeth. His study was performed on extracted single rooted teeth. The method is based on two dental features, namely periodontosis and root transparency. Periodontosis was defined as gingival regression due to the degeneration of soft tissues surrounding the tooth. He measured the distance on the facial surface of the tooth from the cementoenamel junction to the line of soft tissue attachment (or periodontal fibers) on the root. Transparency of the root is a physiological feature that is due to age-progressive deposition, within the dentine tubules, of crystals of hydroxyapatite. With the help of a negatoscope, Lamedin and coworkers were able to measure the length of this transparency from the apex to its highest point on the root. This method was compared to Gustafson's (1950) classic method, which uses six features of dental microstructure, and is still considered by most forensic science textbooks as the reference dental method of determining age at death in adults. The current method had a lower mean error for the estimated of age than the Gustafson method, and the Lamedin method does not require special equipment or training. The measurements are made on the labial surface of the entire tooth without sectioning that would destroy the specimen for further evaluation. 


\section{Bilateral Asymmetry in the Permanent Teeth}

The existence of size asymmetry within the dentition has long been recognized. A tooth on one side of the mouth may be larger or smaller than its antimere on the other side by up to some tenths of a millimeter (e.g., Ballard 1944; Bolton 1958; Lundström 1961; Moorrees and Reed 1964). Garn and coworkers (1966) reported that bilateral asymmetry in the permanent dentition showed no systematic tendency toward sidedness on a group basis. Also, the more distal the tooth (e.g., the tooth farther from the midline) of each morphological class (i.e., incisor, premolar, and molar) is subject to disproportionate size asymmetry compared to the mesial, stable tooth. Larger teeth are subject to greater bilateral discrepancies in tooth size. In addition, third molar agenesis resulted in greater size asymmetry throughout the dentition. Garn et al. (1966) concluded that intraclass size asymmetries (e.g., central and lateral incisors are in the same class) are positively correlated, but interclass asymmetries (e.g., such as likenesses between incisors and premolars), even though involving adjacent teeth, are not systematically related.

\section{Tooth Formation and Eruption Patterns}

Teeth begin to erupt when their roots are about half formed, though there is variation both within and among tooth types, with the incisor root 
generally being less developed (Moyers 1988). In both the primary and permanent dentitions, the mandibular central incisors erupt earlier than their maxillary counterparts (e.g., Steggerda and Hill 1942; Debrot 1969; Tanguay et al. 1984).

The emergence of the permanent incisors usually begins at 6 to 7 years of age with the mandibular central incisor, followed by emergence of the mandibular lateral incisor and maxillary central incisor at about 7 to 8 years. The maxillary lateral incisor is last to emerge at about 8 to 9 years (Hurme 1949). Work by Hurme (1949) on the normal ranges of permanent tooth emergence was intended to aid the pedodontist and general practitioner when assessing whether a given child is fast or slow in obtaining his permanent teeth. Hurme found some 24 articles that contained usable data dealing with the emergence times of permanent teeth, and he derived "universal" standards of tooth emergence for White children living in the northern temperate zone. Hurme's data have been extensively reproduced in dental textbooks (e.g., Wheeler 1974).

Suda et al. (2002) studied the relationship between formation and emergence of maxillary teeth and the skeletal pattern of the maxilla. The study consisted of Japanese patients with maxillary retrusion resulting in a Class III malocclusion compared to a control group with normal occlusion. Their findings indicated that the formation of the teeth progresses as age increases, 
but the skeletal pattern of the maxilla does not allow prediction of tooth formation. They reported that the posterior portion of the maxilla's reduced Sagittal dimension in Class III patients may account for the delay in maxillary second molar eruption. That is, the maxillary retrusion group had significantly delayed second molar emergence compared to the control group. Haruki et al. (1995) have also reported a positive association between palatal length and timing of maxillary posterior tooth emergence. 


\section{CHAPTER III}

\section{MATERIALS AND METHODS}

\section{Materials}

The sample consists of 148 adolescents, 51 males and 97 females, examined between the ages of 9 to 19 years of age. They are American Whites living in the vicinity of Jonesboro, Arkansas. The sample consists of selected pretreatment orthodontic cases from a private practice orthodontist. We measured the pretreatment periapical radiographs for the permanent maxillary and mandibular incisors.

There are four main criteria for inclusion in this study:

1. Patient's dental radiographs (FMS) were taken prior to any orthodontic treatment (also excluding partial treatment administered by general dentists or pediatric dentists). We are assuming these radiographs were exposed using a standardized paralleling technique. We do know that these FMS came from different general dentists in the surrounding areas.

2. Patients are American White as gauged from the patient's extraoral photographs. The intent here is simply to remove the effects of racial variation that are known to affect tooth size, arch size and shape, and type of malocclusion (e.g., Kieser 1990; Harris and Rathbun 1991).

3. Presence of full-mouth dental casts of the patient taken synchronously 
with the FMS. Caliper-measurements of the incisors on the casts were used to adjust for magnification of the periapical (PA) film images.

4. Incisors were excluded, on a tooth-by-tooth basis, if, from inspection of the casts, they are too deviated to provide a reasonably oriented PA film image. Statistical analysis adapts to missing variables within a tooth type as well as missing teeth within a subject. Also, obvious improper radiographic techniques resulting in elongation or foreshortening of the incisor teeth were excluded. It has been reported that minimal geometric and vertical distortion of radiographic images occurs with the use of a standard paralleling technique (Langland et al. 1984, Goaz and White 1987). There may be more difficulty in obtaining a properly exposed mandibular anterior PA than one for the maxillary anterior. Local factors (e.g., tongue, narrowness of the mandible, and tenderness of the floor of the mouth) may contribute to operator variability in exposing a lower radiograph. One may argue that the curvature of the palatal vault, proclination of the incisors, or hyperactive gag reflex could make a maxillary anterior PA equally difficult to the mandibular (Couch, personal experience).

All incisors were fully erupted with their root apices closed (fully formed) by this age (Liversidge 2003; Harris and McKee 1990). Tooth roots were not measured if they did not meet these two criteria. 
These are a sample of convenience. We made an effort to collect representative sample sizes of patients with Angle's Class I, Class II, and Class III malocclusions.

\section{Methods}

We measured the periapical radiographs using a computer assisted photogrammetric method. PA radiographs were scanned in grey scale on a trans-illuminating flatbed scanner at 2,500 dpi. Scans included a millimetric scale to control for image magnification. The digitized tooth landmarks are illustrated in Figures 1 through 3:

1. Point A: Apical-most limit of the root, approximating the center of the apical foramen.

2. Point B: Incisal-most limit of the crown, gauged in the middle third of the incisive edge.

3. Point C: Distal-most aspect of the distal margin of the crown. In proper occlusion, this corresponds to the anatomic contact with the adjacent tooth. It also is the point at the crown's height of contour.

4. Point D: Mesial-most aspect of the mesial margin of the crown. In proper occlusion, this corresponds to the anatomic contact with the adjacent tooth. It also is the point at the crown's height of contour. 


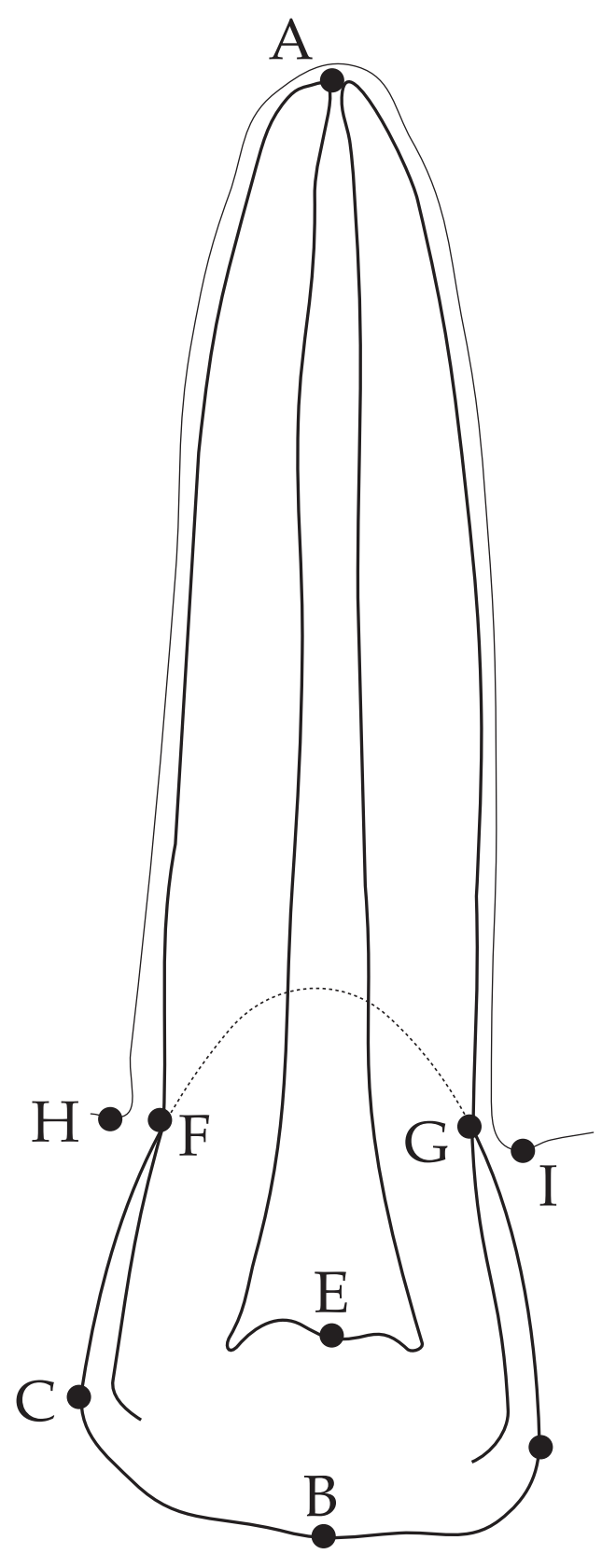

Fig. 1. Diagrammatic faciolingual radiographic view of a maxillary central incisor, showing the points digitized. 


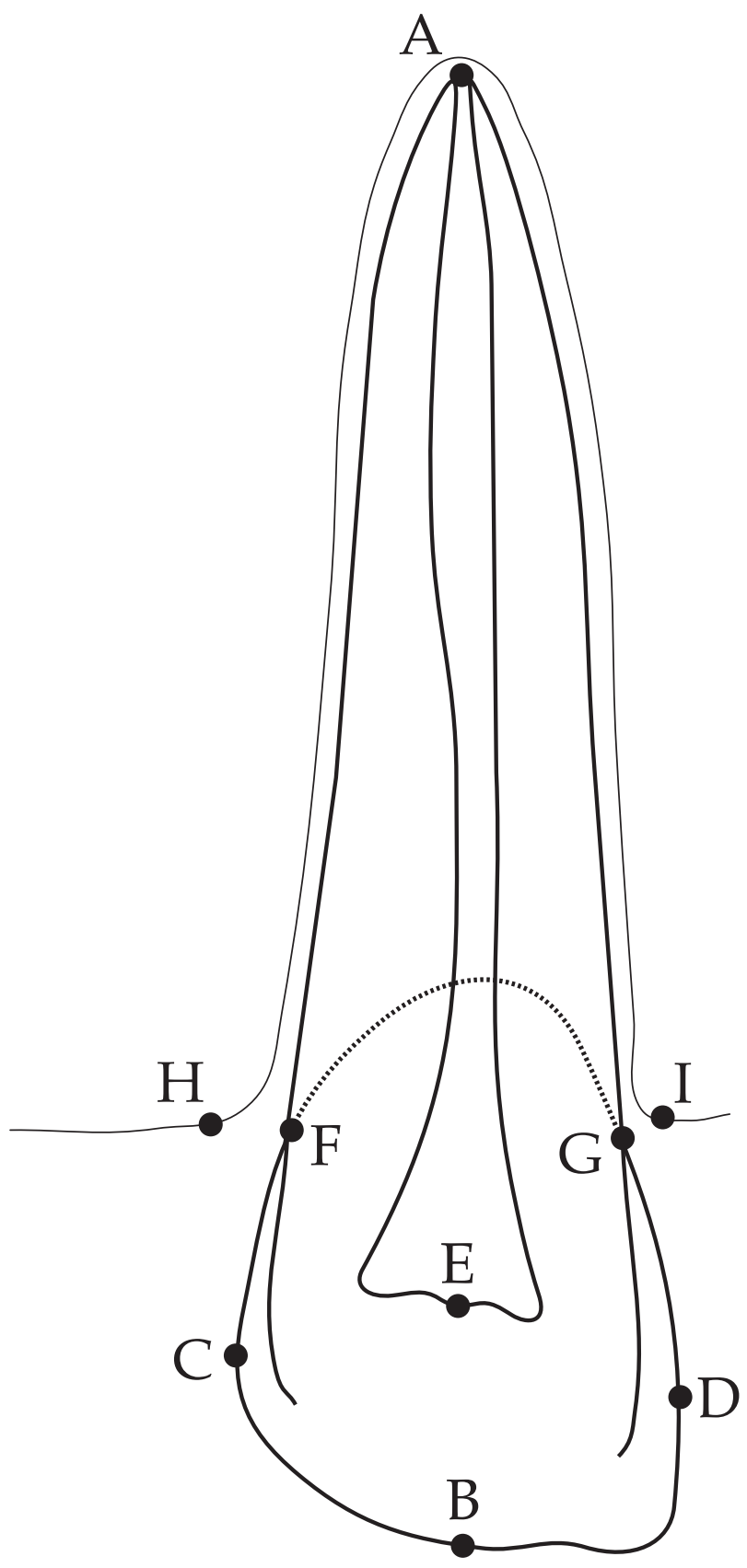

Fig. 2. Diagrammatic faciolingual radiographic view of a maxillary lateral incisor, showing the points digitized. 


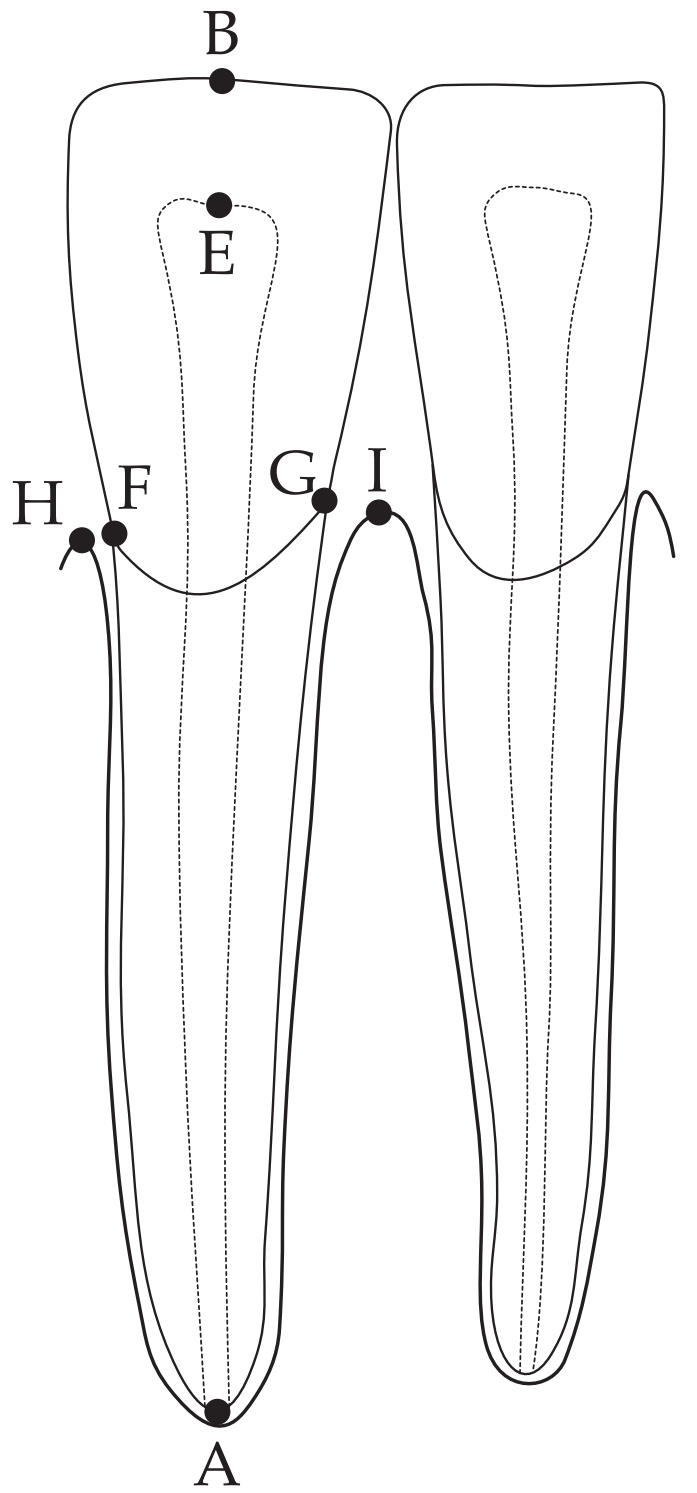

Fig. 3. Diagrammatic faciolingual radiographic view of mandibular central and lateral right incisors, showing the points digitized. 
5. Point E: Coronal-most limit of the pulp chamber gauged in the medial third of the crown, not necessarily corresponding to the occlusal height of the pulp horns.

6. Point F: Distal limit of the cementoenamel junction on the distal aspect of the tooth.

7. Point G: Mesial limit of the cementoenamel junction on the mesial aspect of the tooth.

8. Point H: Coronal-most limit of the crestal bone adjacent to the distal aspect of the tooth.

9. Point I: Coronal-most limit of the crestal bone adjacent to the mesial aspect of the tooth.

\section{Derived Variables}

Numerous data could be generated from this analysis, but, for our purposes, the following five variables were measured.

1. Mesiodistal crown width. This is particularly relevant, since it was used to adjust for magnification, at least in the transverse plane. That is, mesiodistal width of each incisor was obtained using sliding calipers from the dental cast of each person, and this "real" width was used to correct for magnification of the radiographic images. (We are aware that axial inclinations of the tooth to the film and of the source to the tooth can affect 
the transverse and the coronogingival dimensions of the tooth. We are unaware of a method, after the fact, to correct for coronogingival distortions, and we depended, instead, on the operator's experience and ability to correctly orient the source and film with the tooth. Our experience is that methods such as Dermaut and De Munck (1986) create more noise than true axial correction.)

2. Crown height. This is the difference in overall tooth length minus the root length (Figure 1).

3. Root length. This is the straight-line distance from Point $A$ to Point $G$ and separately from Point A to Point $\mathrm{H}$, so a mesial and distal root length was calculated for each tooth type.

4. Crown/root ratio. The ratio of crown height divided by root length, expressed as percentage.

5. Crestal bone height. This is the straight-line distance from Point A to Point $\mathrm{H}$ and separately from Point $\mathrm{A}$ to Point I, so a mesial and distal crestal bone height was calculated for each tooth type.

6. Pulp Height. This is the distance from Point A to Point E. 


\section{$\underline{\text { Data Acquisition }}$}

The procedure for data collection is this:

1. Demographic data (sex and age) were entered into an Excel® data file. The tooth size dimensions were collated here as well. These digitized measurements included both mesial and distal root lengths, mesial and distal alveolar crestal bone heights, tooth length, crown width, and pulp height of a selected UI1, UI2, LI1, and LI2 per subject. Crown widths of all eight incisors measured with digital-readout sliding caliper were also recorded to the nearest $0.01 \mathrm{~mm}$.

2. Each periapical film was scanned on a flatbed scanner at 2,500 dpi (256 grey scale). The scans included millimetric grids along the sides of the films so that magnification was controlled. In addition, the sizes of the periapical films (measured with sliding calipers) were known. Images were saved as TIFF files.

3. SPSS SigmaScan Pro 5.0 was used to digitize the films (i.e., locate the landmarks as Cartesian coordinates) and to generate the desired distances. The data file for each case were transferred and merged into one spreadsheet file.

4. Statistical analysis was performed using the JMP 5.1.2 statistical package (SAS Institute, Cary, NC). 


\section{Statistical Analysis}

The questions asked of these data are fairly straightforward; one batch of issues is purely descriptive, namely, by Angle's classification and/or sex, what are the normative dimensions of the teeth? What are the size differences between the incisor tooth types? How much left-right asymmetry is there? A second battery of questions involves understanding and partitioning the variation in the sample. As examples: How much sexual dimorphism is there, and does this differ between crown and root dimensions?

Descriptive statistics were calculated as defined by Sokal and Rohlf (1995), namely sample size (n), arithmetic mean $(\bar{x})$, standard deviation (sd), sample variance $\left(\mathrm{s}^{2}\right)$, standard error of the mean (se), skewness $\left(\mathrm{g}_{1}\right)$, and kurtosis $\left(\mathrm{g}_{2}\right)$. Regarding skewness and kurtosis, statistical packages commonly fail to provide inferential tests of whether $\mathrm{g}_{1}$ or $\mathrm{g}_{2}$ differ significantly from normality. Inspection of these raw statistics themselves is not particularly informative. Following Sokal and Rohlf (1995, p 138), the standard error for skewness is

$$
\mathrm{se}_{\mathrm{g}_{1}}=\sqrt{\frac{6 \mathrm{n}(\mathrm{n}-1)}{(\mathrm{n}-2)(\mathrm{n}+1)(\mathrm{n}+3)}}
$$

where $\mathrm{n}$ is the sample sizes, and the standard error for kurtosis is

$$
\mathrm{se}_{\mathrm{g}_{2}}=\sqrt{\frac{24 \mathrm{n}(\mathrm{n}-1)^{2}}{(\mathrm{n}-3)(\mathrm{n}-2)(\mathrm{n}+3)(\mathrm{n}+5)}}
$$


An interesting feature of the tests of whether skewness or kurtosis departs from normality is that they are each evaluated at infinite degrees of freedom regardless of the actual size of the samples.

Exploratory data methods (Tukey 1977) were used to identify statistical outliers. Analysis of variance (one-way and mixed-model) was used to address the questions just listed, using designs described by Winer et al. (1991). A model commonly employed is the two-way factorial analysis of variance, where sex is included as one of the factors to control for the well-documented sexual dimorphism in tooth dimensions. The paired t-test was used to test for size differences in homologous left-right (or mesial-distal) dimensions.

Percentage sexual dimorphism was calculated from this formula:

$$
\left(\frac{\left(\bar{x}_{M}-\bar{x}_{F}\right)}{\bar{x}_{F}}\right) 100
$$

so the percentage is read as the degree to which the male average exceeds the mean size of females.

Pearson product-moment correlation coefficients ( $\mathrm{r}$ ) were used to measure the strengths of associations between variables. Principal components analysis was performed using the covariance matrices (Cooley and Lohnes 1971; Harman 1976).

Statistics were generated using JMP version 5.0.02 (SAS Institute Inc., Cary, NC). Tests were two-tail, and the conventional level of statistical 
significance (alpha $=0.05)$ was used throughout.

\section{Research Goals}

There are a variety of applications for these normative data. We tested several specific hypotheses:

1. Prior studies claim that there is appreciably greater sexual dimorphism in root size than crown size (e.g., Garn et al. 1978a). This was tested and could prove useful for forensic studies (e.g., Ditch and Rose 1972; Bowers and Bell 1995).

2. Crown-root size differences were tested among types of malocclusions. Data suggest that there are sex differences in crown-root proportions (e.g., Stramotas et al. 2000; Holtta et al. 2004), but radiographic distortions were not controlled for in those studies. Data also suggest (e.g., Cua-Benward et al. 1992; Basdra et al. 2000) that there may be differences in these ratios among types of malocclusions (Angle), but the available data are hardly compelling, and the question needs retesting.

3. We do not know whether the age interval is sufficient to see the anticipated increase in crestal bone height with age that is supposed to occur during adolescence (Darling and Levers 1975), but we will test for it. 
It is beyond the scope of the present research project, but the data collected here will be valuable for defining the prevalence and extent of Bolton discrepancies in a sample of orthodontic patients (Bolton 1959). A Bolton discrepancy is an imbalance between the sum of mesiodistal incisor widths in the two jaws so that, simply, the teeth cannot couple properly between the arches (e.g., Saatci and Yukay 1997; Araujo and Souki 2003). A second application involves the question of the degree to which tooth size per se contributes to crowding (e.g., Lavelle 1972; Howe et al. 1983). That is, is dental crowding caused by larger teeth? 


\section{CHAPTER IV}

\section{RESULTS}

\section{Evaluation of Normality}

Because the cases studied here were drawn from a sample of orthodontic patients, one expects that some individuals will present with aberrant tooth dimensions (e.g., Bolton 1959). The current data were, then, culled to eliminate statistical outliers. This was done at two levels, (1) cases with visually obvious morphological issues were omitted and (2) the sex-specific distributions of each variable were scrutinized to omit severe outliers. The skewness and kurtosis were reviewed for each variable after culling to assure that the distributions did not depart from normality. Tables 1 and 2 list the first four moments of the distribution for each variable, specifically whether skewness and kurtosis are supportive of a normal distribution. Skewness (gamma-one, $\mathrm{g}_{1}$ ) is a measure of left-right asymmetry of the distribution. The sign of $\mathrm{g}_{1}$ indicates which tail of the distribution that is extended; a positive sign indicates that the right end of the distribution is extended (positive skewness), and a negative sign shows that the left end of the distribution is larger (negative skewness).

After culling statistical outliers (omission by variable, not case) resulted in all distributions conforming to normality (Tables 1,2). 


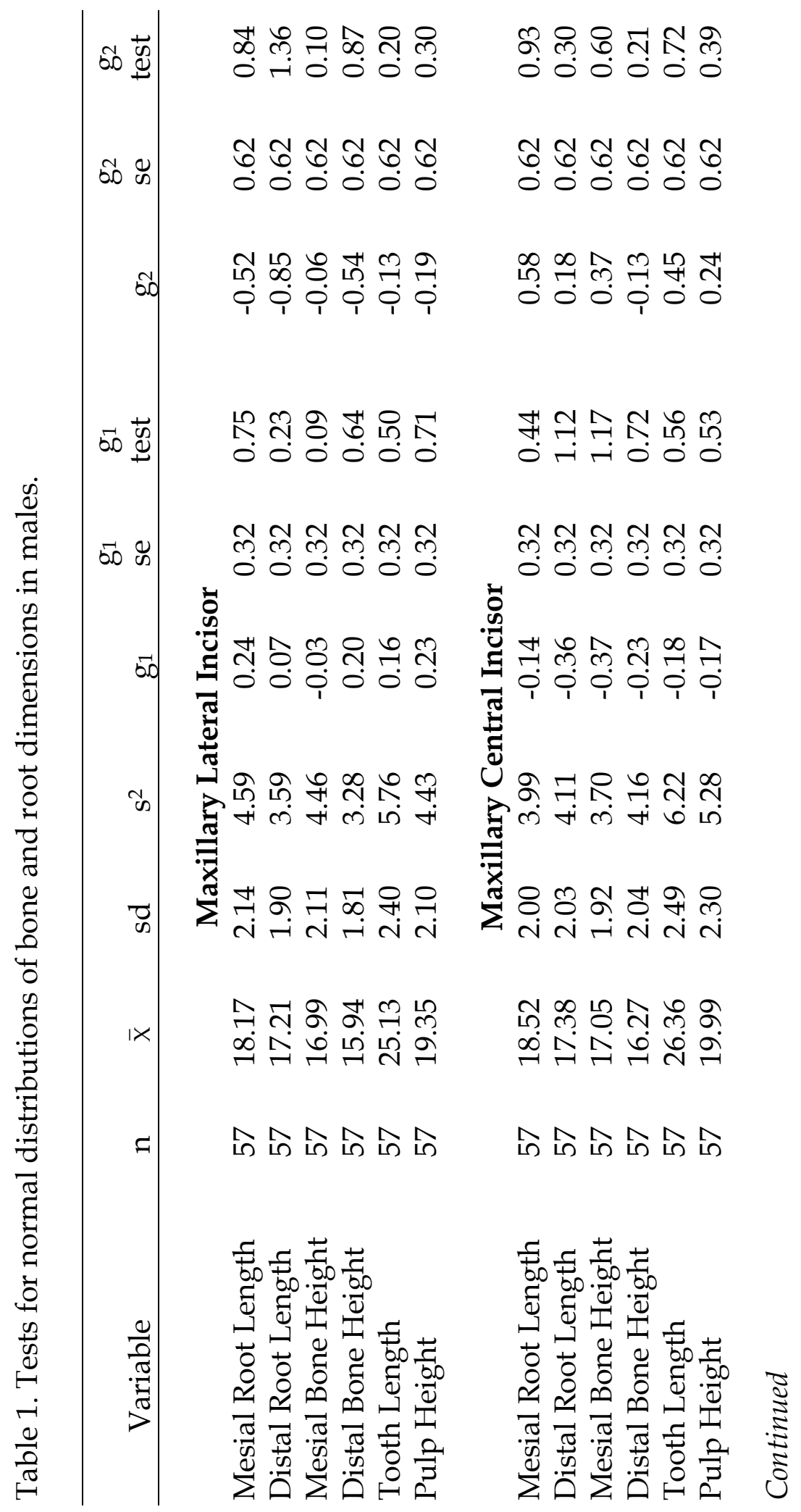




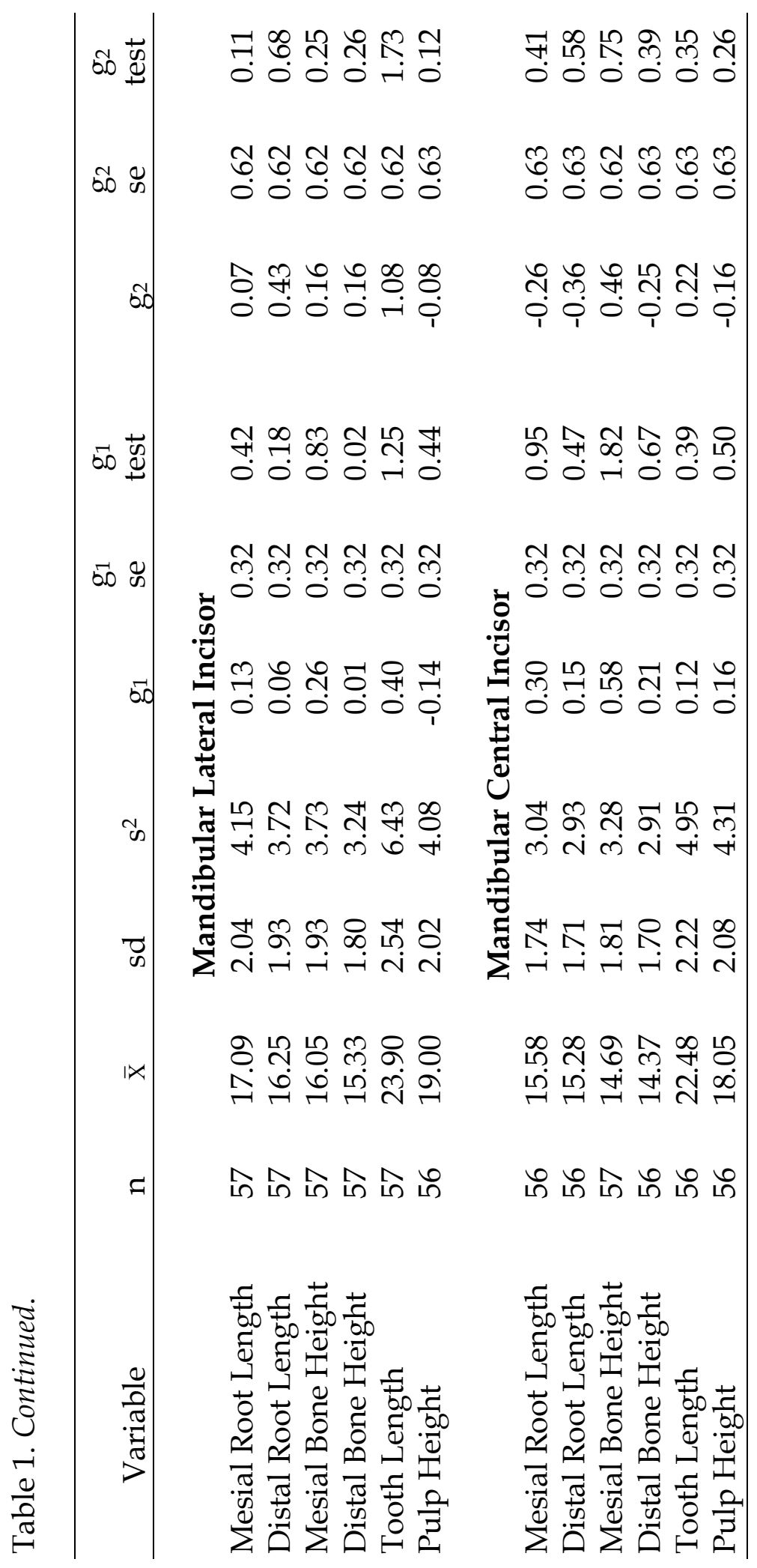




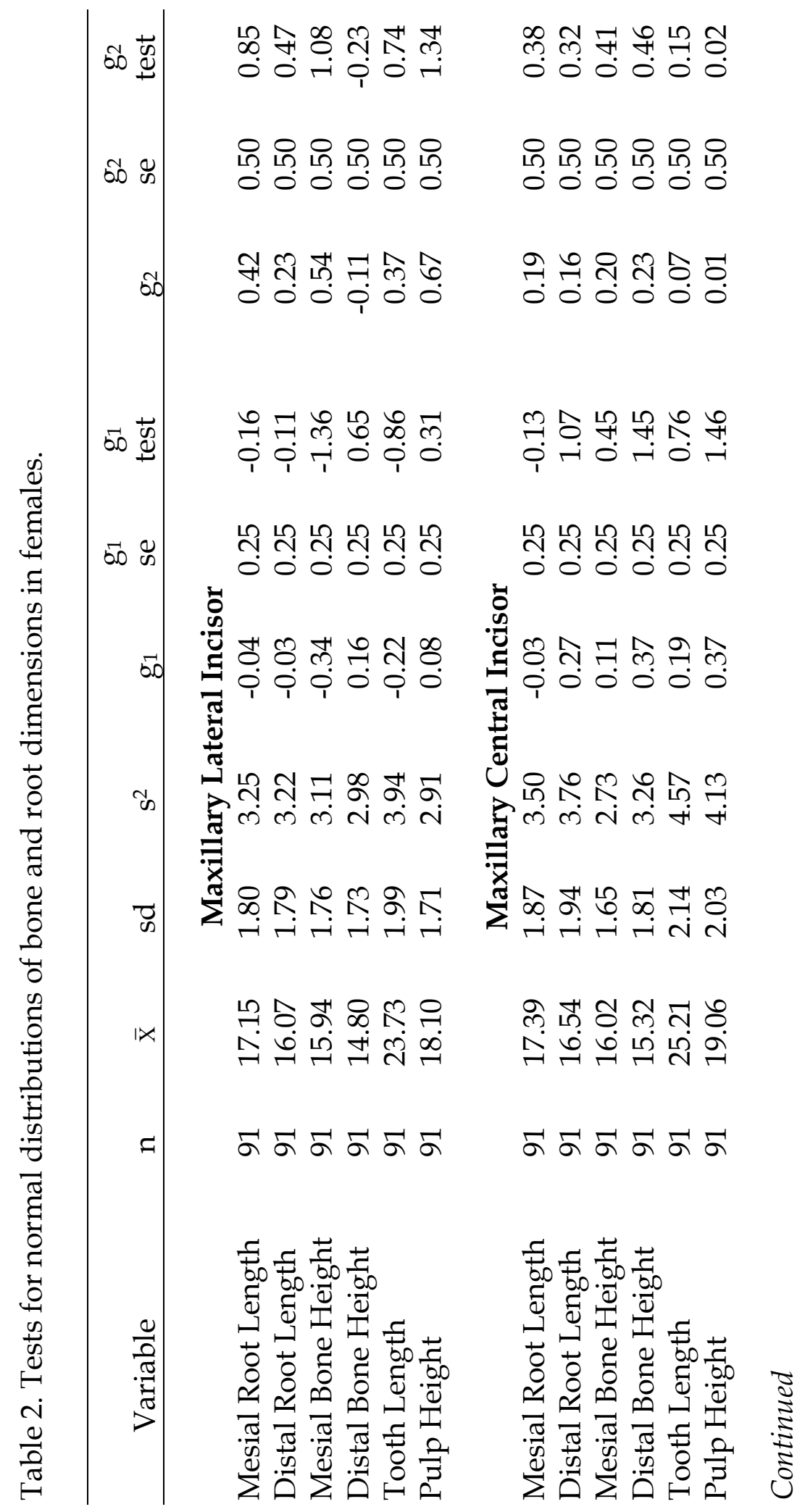




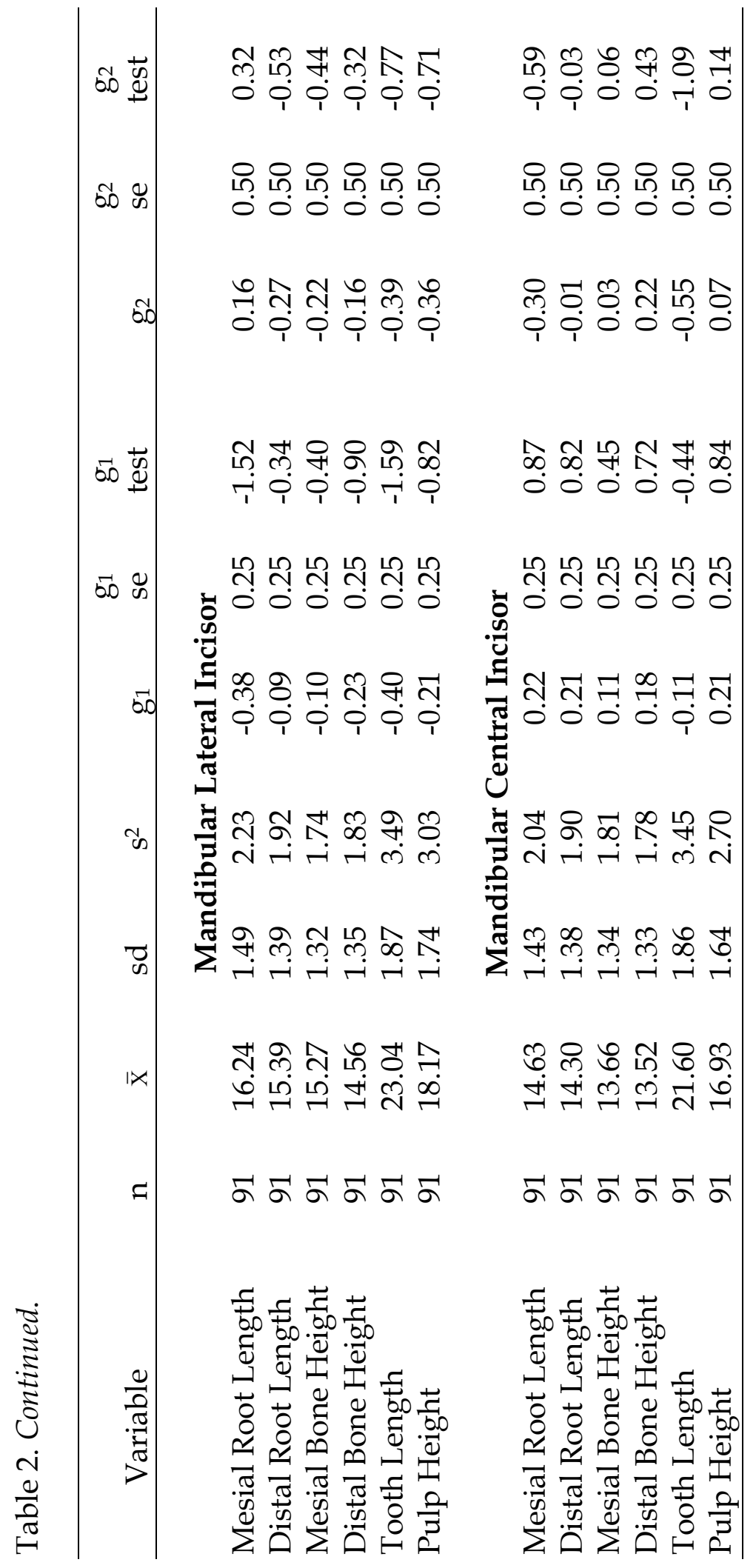


Kurtosis (gamma-two, $\mathrm{g}_{2}$ ) refers to the "peakedness" of a distribution. If kurtosis is zero, kurtosis of the distribution is normal. The larger the value of $\mathrm{g}_{2}$, the flatter (platykurtotic) the distribution, and when the statistic is negative, larger values show that the distribution is more leptokurtotic - more peaked than a normal distribution. The data were culled for statistical outliers as regards kurtosis, and the culled results are listed in Table 1 (males) and Table 2 (females).

The data in Table 1 and Table 2 are derived from a photogrammetric method employed on the periapical radiographs. The mesiodistal crown diameters of the incisors were measured on the associated dental casts using sliding calipers in order to correct for mediolateral distortions in sizes of the tooth images on the radiographs. Descriptive statistics are listed in Table 3 (males) and Table 4 (females). Again, the data for each variable have been culled to eliminate statistical outliers, as confirmed by the nonsignificant tests for skewness and kurtosis. These culled data were used for all subsequent analysis.

\section{Left-Right Symmetry}

Root length and bone height were measured on both left and right sides of the incisors, which affords the opportunity to test for systematic side 


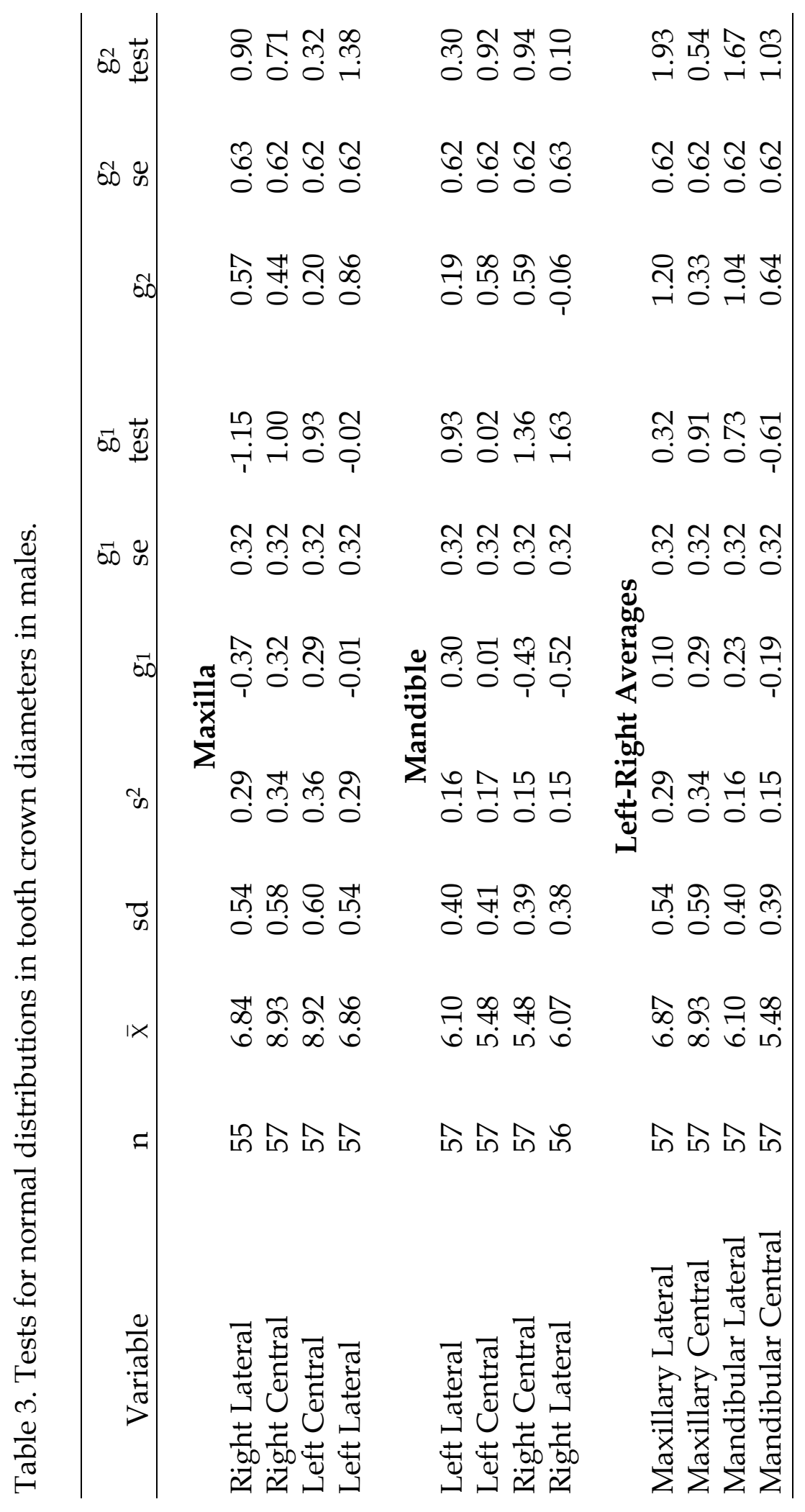




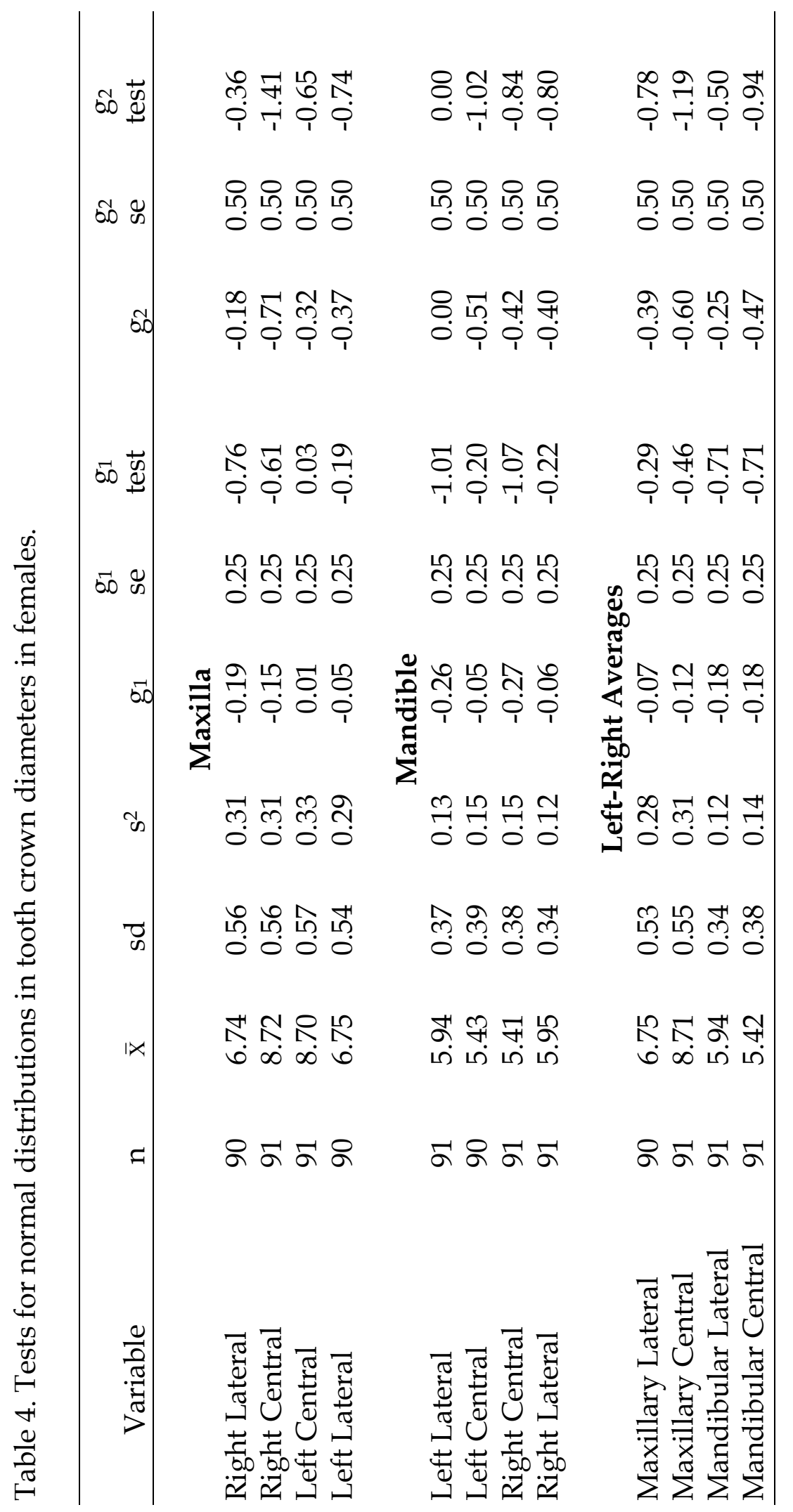


differences. Root length was measured as the distance from the root apex to the CEJ on the mesial side of the tooth and then separately from the root apex to the CEJ on the distal side of the tooth. Bone heights, comparably, were measured as the distance from the root apex to the crestal bone on the mesial side of the incisor and, independently, to the distal side. To minimize the effect of foreshortening or elongation of the teeth on the periapical radiographs due to variations in the x-ray beam projections, the interproximal alveolar bone height measured from the radiograph is often expressed as a percentage of the root or tooth length (e.g., Schei et al. 1959; Bjorn and Holmberg 1966; Jeffcoat and Williams 1984; Goodson et al. 1984; Kullman and Martinsson 1985; Lavstedt et al. 1986).

If the facial morphologies of the incisors were perfectly symmetric, then the mesial and distal dimensions should be very similar, but, of course, lateralities in the crowns and roots are distinctive in the left and right quadrants of an arch (Zeisz and Nuckolls 1949; Ash 1993). That is, characteristic asymmetries generally make it easy to identify whether an extracted tooth is from the left or right quadrant. On inspection (Table 5), there is a consistent laterality across all four incisors tooth types as regards root length and alveolar crestal bone height: distal dimensions are systematically shorter than the corresponding dimensions on the mesial side of the tooth. 


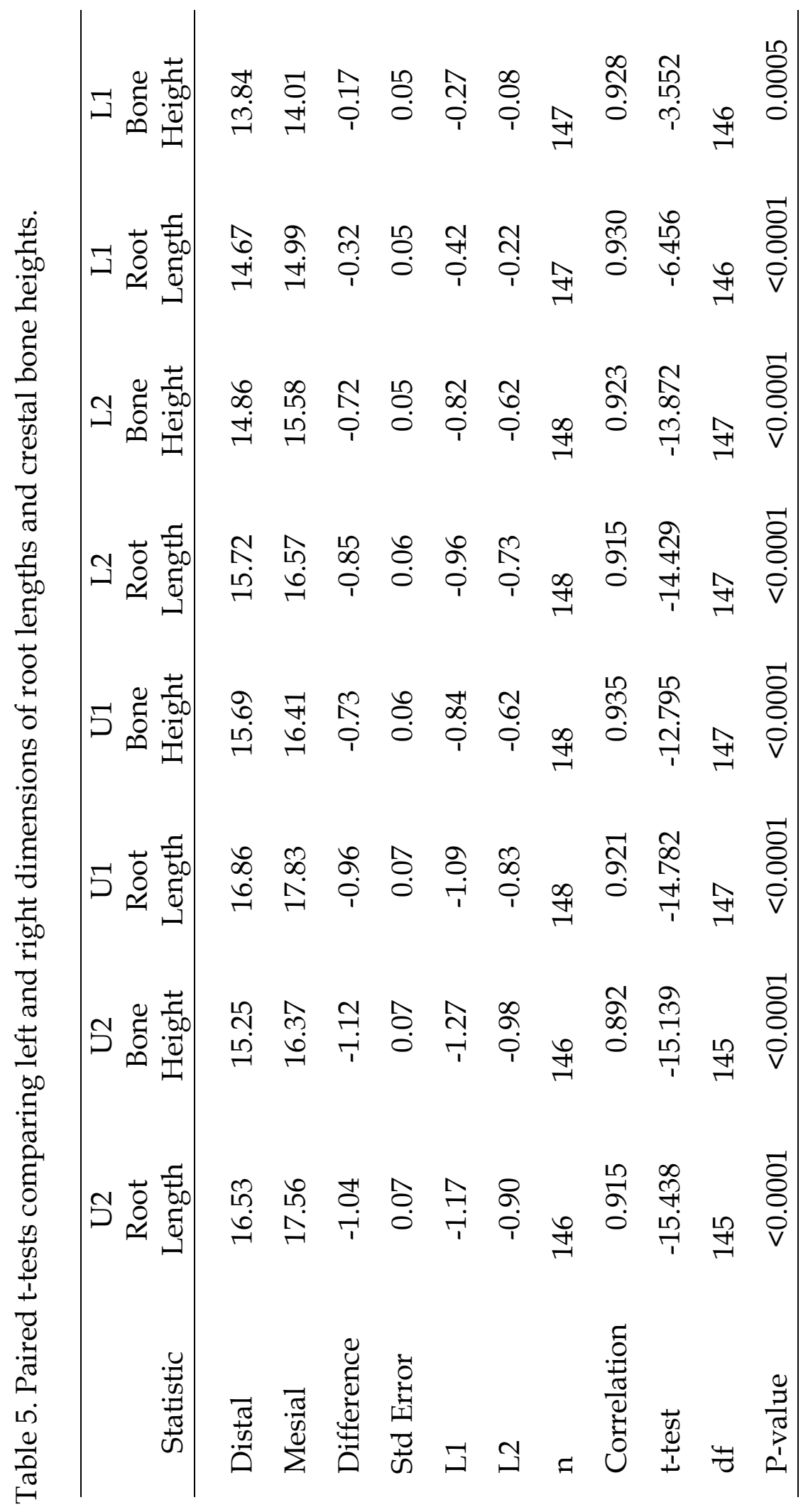


These differences are highly significant statistically. The differences between sides are on the order of a millimeter in the maxilla, but less in the mandible, especially for the central incisors.

For example, root length on the maxillary central incisor is $16.9 \mathrm{~mm}$ when measured on the distal aspect, but $17.8 \mathrm{~mm}$ on the mesial aspect, a difference of about $1 \mathrm{~mm}$. This could be due to the distal deflection of the apical third of the roots (e.g., Ash 1993). That is, incisor roots are not perfectly straight; the apical region in particular often curves to the distal as this narrowest region of the root follows the neurovascular triad's path distally-and-superiorly. Likewise, for bone height on this tooth, the mean distance is $15.7 \mathrm{~mm}$ on the distal but 16.4 $\mathrm{mm}$ on the mesial. Again, the difference $(\bar{x}=0.7 \mathrm{~mm})$ shows that crestal bone heights are systematically greater on the mesial sides of the incisors. Again, this asymmetry may be due to the measurement method. We simply used the straight-line distance between landmarks, so the greater bone heights may merely reflect distal-deflection of the incisor's root.

Table 6 lists comparable tests for left-right differences in the mesiodistal incisor crown dimensions measured from the dental casts with calipers. In the prior tests (Table 5) the question was whether the tooth itself was symmetric. In Table 6, the question is whether homologous between teeth in the left and right quadrants are metrically the same. There is no suggestion of sidedness in 
Table 6. Paired t-tests comparing side differences in mesiodistal crown dimensions.

\begin{tabular}{lcccc}
\hline Statistic & $\begin{array}{c}\text { Maxillary } \\
\text { Lateral } \\
\text { Incisor }\end{array}$ & $\begin{array}{c}\text { Maxillary } \\
\text { Central } \\
\text { Incisor }\end{array}$ & $\begin{array}{c}\text { Mandibular } \\
\text { Lateral } \\
\text { Incisor }\end{array}$ & $\begin{array}{c}\text { Mandibular } \\
\text { Central } \\
\text { Incisor }\end{array}$ \\
\hline Left & 6.79 & 8.78 & 6.00 & 5.45 \\
Right & 6.79 & 8.80 & 6.01 & 5.43 \\
Difference & 0.00 & -0.02 & 0.01 & 0.01 \\
Std Error & 0.03 & 0.02 & 0.02 & 0.01 \\
L1 & -0.05 & -0.05 & -0.03 & -0.01 \\
L2 & 0.05 & 0.02 & 0.04 & 0.04 \\
$\mathrm{n}$ & 146 & 148 & 148 & 147 \\
Correlation & 0.848 & 0.932 & 0.865 & 0.911 \\
t-test & 0.112 & -1.105 & 0.425 & 1.020 \\
df & 145 & 147 & 147 & 146 \\
P-value & 0.9108 & 0.2712 & 0.6712 & 0.3092 \\
\hline
\end{tabular}


the crown dimensions; indeed, all of the correlation are high (ca. 0.8 to 0.9 ), and the mean differences are effectively zero.

\section{$\underline{\text { Sexual Dimorphism }}$}

It is well documented that tooth dimensions are, statistically, larger in males than females even though humans are the least dimorphic of the great apes (Garn et al. 1967; Swindler 2002). This principle easily extends to the present data (Table 7). The percentage sexual dimorphism was calculated from the sex-specific means as $(\mathrm{M}-\mathrm{F}) / \mathrm{F}$, so the percentages can be read as the degree to which the sex difference exceeds the female mean. These percentage differences are in the neighborhood of 4 to $7 \%$, and inspection suggests that the root dimensions are more dimorphic than the mesiodistal crown dimensions shown in Table 8. This difference has been investigated in more detail in Harris and Couch (2006).

Six tooth and alveolar dimensions are measured for each tooth type (Table 7), and for each of the 24 variables, males have significantly larger mean sizes than females. These significant results emphasize the need to account for size differences in the assessment of other variables.

Table 8 lists the results of tests for sexual dimorphism for the mesiodistal crown dimensions. Here, again, one is struck by the diminished sexual dimorphism compared to the root variables tested in Table 7. Here the 


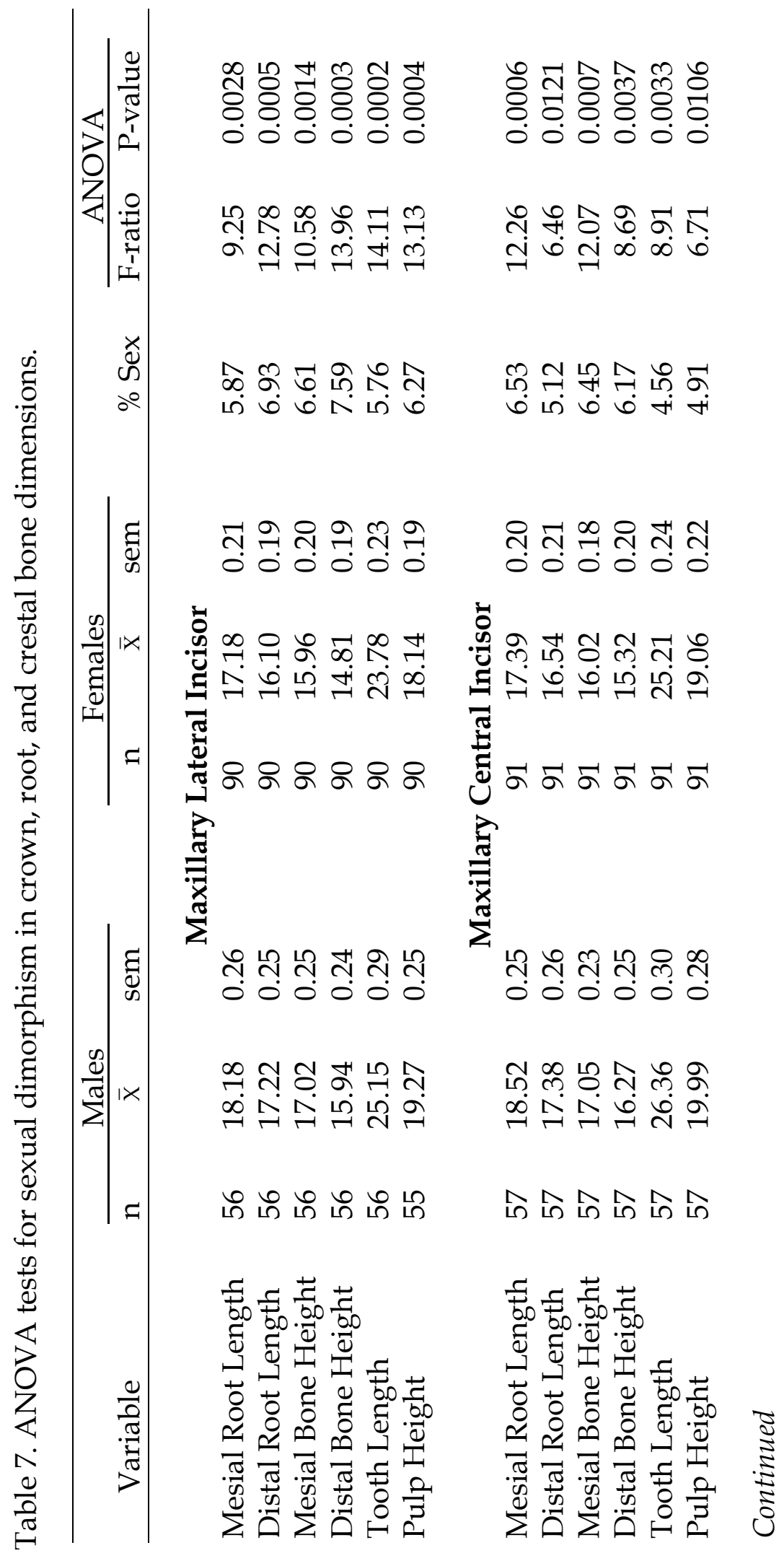




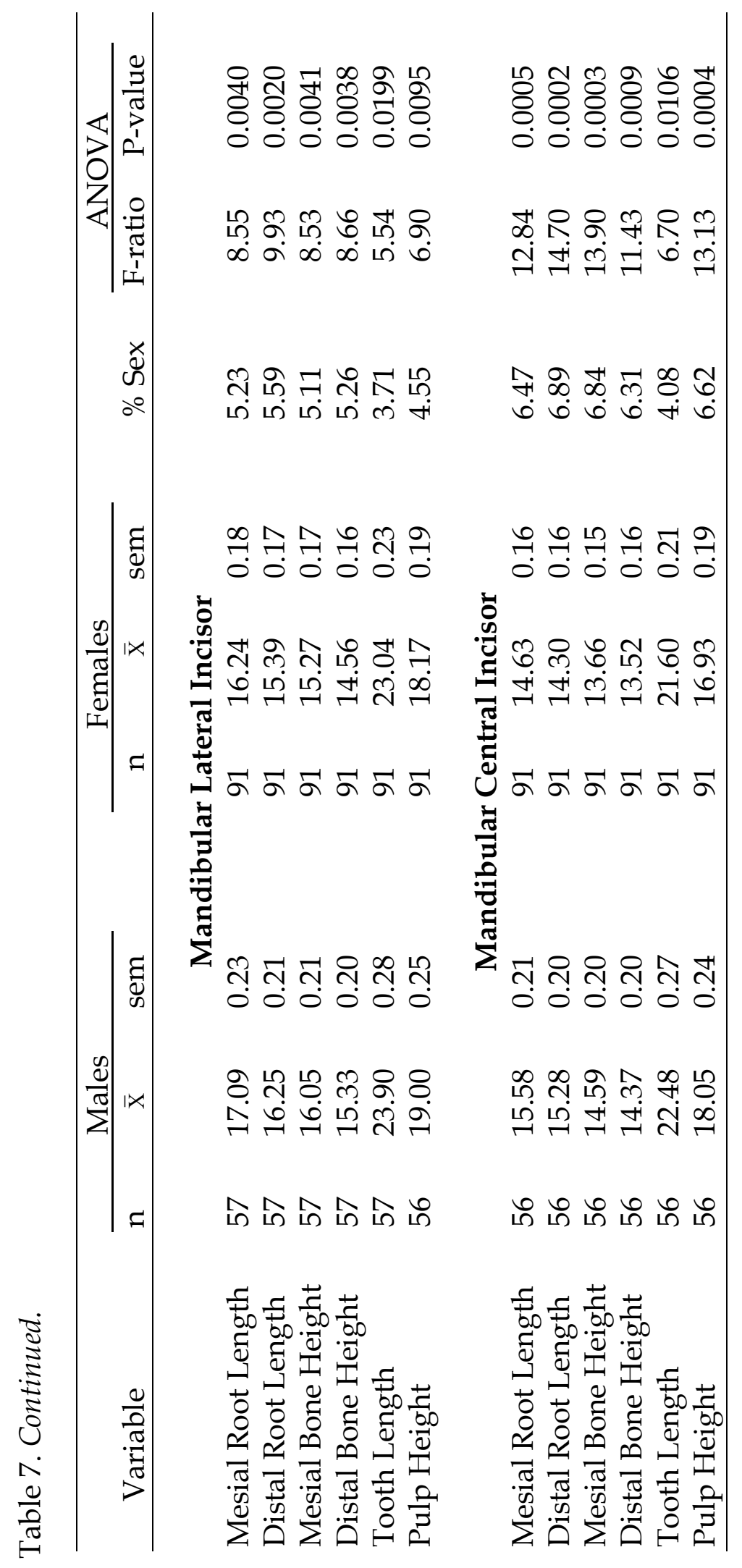




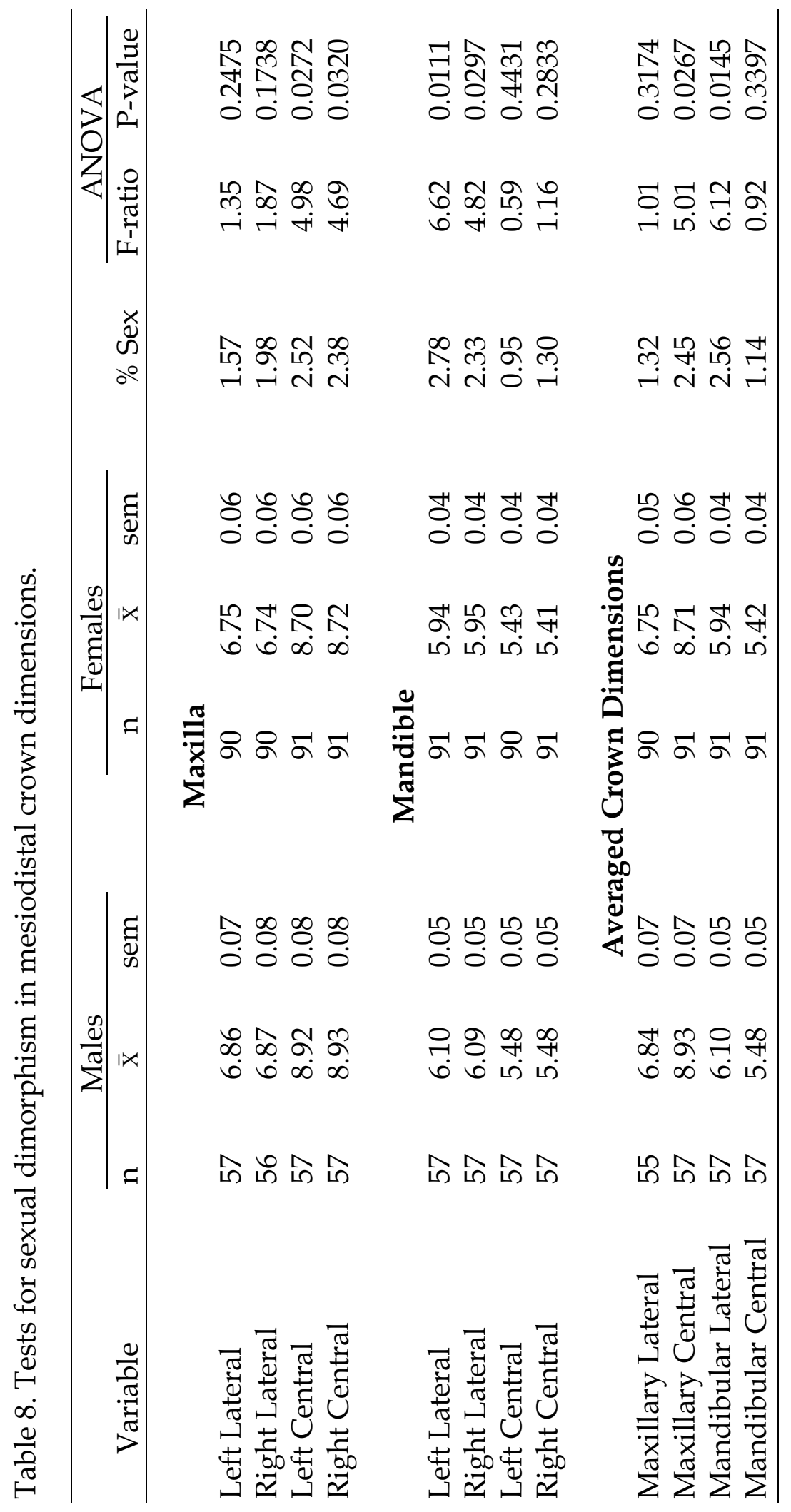


statistical results show that males are not statistically larger than females. Indeed, just six of the 12 tests reach statistical significance (alpha $=0.05)$, and the significant variables differ by arcade. For the left-right averages, just the maxillary central and mandibular lateral incisor tooth types exhibit significant dimorphism, and here the percentage sexual dimorphism is only on the order of 2 to $3 \%$.

\section{Effects of Angle's Classification}

Inspection of the patients' diagnostic records allowed classification according to Angle's class, namely buccal segment relationships were Class I, II, or III. Of the cases classified $(n=148)$, most were Class I $(96 / 148 ; 64.9 \%)$ or Class II (43/148; 29.0\%). Just 9 cases were Class III (9/148; 6.1\%).

The Class III cases were ignored as too few to be informative, and the dental variables were tested for size differences between Class I and II. "Sex" was included in the models to take account of the differences noted previously, making the tests two-way factorial analyses of variance.

Results of the ANOVA tests for the tooth components are listed in Table 9, and there are scattered statistically significant differences between Class I and II cases in the maxilla, particularly for the lateral incisor. Of note, three of the six variables for $\mathrm{U} 2$ are significant at the conventional level of alpha, and the other three are different at $0.10>\mathrm{P}>0.05$. In passing, it is evident, as 
Table 9. Results of two-way analysis of variance, testing for size differences by Angle's classification while controlling for patient's sex. ${ }^{1}$

\begin{tabular}{|c|c|c|c|c|c|c|}
\hline \multirow[b]{2}{*}{ Variable } & \multicolumn{2}{|c|}{ Angle Class } & \multicolumn{2}{|c|}{ Sex } & \multicolumn{2}{|c|}{ Interaction } \\
\hline & F-ratio & P-value & F-ratio & P-value & F-ratio & P-value \\
\hline \multicolumn{7}{|c|}{ Maxillary Lateral Incisor } \\
\hline Mesial Root Length & 6.64 & 0.0111 & 5.71 & 0.0183 & 0.49 & 0.4849 \\
\hline Distal Root Length & 3.03 & 0.0840 & 7.31 & 0.0078 & 0.16 & 0.6874 \\
\hline Mesial Bone Height & 8.23 & 0.0048 & 5.90 & 0.0165 & 0.77 & 0.3821 \\
\hline Distal Bone Height & 4.80 & 0.0302 & 8.31 & 0.0046 & 0.20 & 0.6546 \\
\hline Tooth Length & 3.17 & 0.0773 & 8.19 & 0.0049 & 0.27 & 0.6045 \\
\hline Pulp Height & 3.39 & 0.0679 & 9.52 & 0.0025 & 0.54 & 0.4652 \\
\hline \multicolumn{7}{|c|}{ Maxillary Central Incisor } \\
\hline Mesial Root Length & 4.70 & 0.0319 & 11.46 & 0.0009 & 1.33 & 0.2502 \\
\hline Distal Root Length & 1.60 & 0.2075 & 5.51 & 0.0204 & 0.59 & 0.4455 \\
\hline Mesial Bone Height & 3.23 & 0.0745 & 14.06 & 0.0003 & 2.78 & 0.0980 \\
\hline Distal Bone Height & 1.29 & 0.2582 & 8.26 & 0.0047 & 0.83 & 0.3641 \\
\hline Tooth Length & 0.54 & 0.4623 & 7.70 & 0.0063 & 0.46 & 0.4977 \\
\hline Pulp Height & 3.04 & 0.0836 & 8.15 & 0.0050 & 1.47 & 0.2275 \\
\hline \multicolumn{7}{|c|}{ Mandibular Lateral Incisor } \\
\hline Mesial Root Length & 1.46 & 0.2284 & 6.23 & 0.0138 & 0.03 & 0.8728 \\
\hline Distal Root Length & 1.55 & 0.2147 & 5.31 & 0.0227 & 0.26 & 0.6082 \\
\hline Mesial Bone Height & 1.58 & 0.2111 & 5.31 & 0.0228 & 0.29 & 0.5888 \\
\hline Distal Bone Height & 1.46 & 0.2288 & 4.01 & 0.0472 & 0.20 & 0.6546 \\
\hline Tooth Length & 1.65 & 0.2009 & 2.00 & 0.1599 & 0.28 & 0.6000 \\
\hline Pulp Height & 3.14 & 0.0789 & 4.66 & 0.0327 & 0.11 & 0.7352 \\
\hline \multicolumn{7}{|c|}{ Mandibular Central Incisor } \\
\hline Mesial Root Length & 1.49 & 0.2243 & 13.47 & 0.0004 & 0.12 & 0.7263 \\
\hline Distal Root Length & 1.02 & 0.3139 & 13.12 & 0.0004 & 0.09 & 0.7679 \\
\hline Mesial Bone Height & 2.51 & 0.1153 & 14.55 & 0.0002 & 0.04 & 0.8350 \\
\hline Distal Bone Height & 0.85 & 0.3586 & 9.49 & 0.0025 & 0.34 & 0.5631 \\
\hline Tooth Length & 0.42 & 0.5167 & 4.62 & 0.0334 & 0.91 & 0.3429 \\
\hline Pulp Height & 0.87 & 0.3530 & 9.85 & 0.0021 & 0.12 & 0.7288 \\
\hline
\end{tabular}

${ }^{1}$ There is $1 \mathrm{df}$ associated with each of the three tests for each variable (row). 
discussed above, that most of the measured variables exhibit significant sexual dimorphism (and that none of the interaction terms achieved significance). The least-squares group means were taken from the two-way ANOVA and plotted for all variables in Appendix. These graphs show (1) that males are, on the average, larger than females and (2) that Class I teeth - notably the maxillary lateral incisors - are smaller than means for the Class II sample. Comparable differences have been observed in prior studies in this laboratory (Harris, pers. comm.), but the causes of these size differences remain speculative.

Mesiodistal crown dimensions also were tested for a difference between Class I and II malocclusions (Table 10). Here, however, none of the 12 analyses disclosed any significant difference by Angle's molar classification. The corresponding least-squares means also are graphed in Appendix. Inspection of the statistical results in Table 10 shows that some of the interaction terms are significant, especially for the maxillary lateral incisor variables. These occur because the mean sizes for females are slightly smaller than those for males in the Class I group, but slightly larger in the Class II group. We attribute these results to sampling fluctuations rather than any biological difference. It is to be expected that the several dimensions of the same tooth type show similar statistical results because of the positive covariances among them (e.g., Moorrees and Reed 1964; Harris and Bailit 1987). In sum, only the maxillary lateral incisor yields substantive evidence of size difference between Angle's 
Table 10. Results of two-way analysis of variance for mesiodistal crown widths, testing for size differences by Angle's classification while controlling for

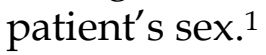

\begin{tabular}{|c|c|c|c|c|c|c|}
\hline \multirow[b]{2}{*}{ Variable } & \multicolumn{2}{|c|}{ Angle Class } & \multicolumn{2}{|c|}{ Sex } & \multicolumn{2}{|c|}{ Interaction } \\
\hline & F-ratio & P-value & F-ratio & $\overline{P \text {-value }}$ & F-ratio & P-value \\
\hline \multicolumn{7}{|c|}{ Maxillary Crown Dimensions } \\
\hline Right Lateral & 0.04 & 0.8380 & 0.03 & 0.8676 & 4.79 & 0.0305 \\
\hline Left Lateral & 0.00 & 0.9946 & 0.04 & 0.8492 & 4.61 & 0.0336 \\
\hline Right Central & 0.03 & 0.8589 & 1.07 & 0.3021 & 3.33 & 0.0702 \\
\hline Left Central & 0.05 & 0.8185 & 1.34 & 0.2487 & 4.06 & 0.0460 \\
\hline \multicolumn{7}{|c|}{ Mandibular Crown Dimensions } \\
\hline Right Lateral & 0.20 & 0.6552 & 3.01 & 0.0851 & 0.29 & 0.5915 \\
\hline Left Lateral & 0.47 & 0.4944 & 3.79 & 0.0537 & 0.70 & 0.4031 \\
\hline Right Central & 0.03 & 0.8728 & 0.30 & 0.5828 & 1.55 & 0.2160 \\
\hline Left Central & 0.04 & 0.8373 & 0.00 & 0.9964 & 3.21 & 0.0755 \\
\hline \multicolumn{7}{|c|}{ Averaged Crown Dimensions } \\
\hline Max Lateral & 0.01 & 0.9156 & 0.03 & 0.8525 & 5.12 & 0.0253 \\
\hline Max Central & 0.00 & 0.9782 & 1.25 & 0.2655 & 3.83 & 0.0526 \\
\hline Mand Lateral & 0.35 & 0.5542 & 3.70 & 0.0566 & 0.52 & 0.4723 \\
\hline Mand Central & 0.00 & 0.9797 & 0.08 & 0.7796 & 2.42 & 0.1222 \\
\hline
\end{tabular}

${ }^{1}$ There is $1 \mathrm{df}$ associated with each of the three tests for each variable (row). 
Classes, with the Class I cases (controlled for sex) having smaller root dimensions than the Class II cases.

\section{Principal Components Analysis}

It is intuitive that dimensions of the crown, root, and supporting bone for a given tooth type are positively intercorrelated because they are all parts of an integrated whole. The purpose of this section is to explore the variancecovariance structure of the maxillary central and the lateral incisors in more detail. The structures were assessed using principal components analysis without matrix rotation (Cooley and Lohnes 1971; Harman 1976).

The pairwise correlation matrix for six variables measured on the maxillary central incisor is listed in Table 11. Review of the correlation coefficients shows (1) that the root and bone variables are highly positively intercorrelated, with correlations of at least 0.8 , but (2) these dimensions are only weakly correlated with mesiodistal crown size. Crown width is only correlated with the six other variables at a level of about $r=0.1$.

Principal components analysis (PCA) for the central incisor is listed in Table 12. Reviewing the eigenvalues, it is apparent that almost all of the shared variance $(91.2 \%)$ is in the first canonical axis. The eigenvectors are positive and subequal for PC One, suggesting that overall size is driving this complex of correlations. On the other hand, none of these PC weights is particularly large 
Table 11. Pairwise correlations for the maxillary central incisor (sexes combined). ${ }^{1}$

\begin{tabular}{llcll}
\hline Variable A & Variable B & Correlation & $\mathrm{n}$ & P-value \\
\hline Dis Root Length & Mes Root Length & 0.9183 & 139 & $<0.0001$ \\
Mes Bone Height & Mes Root Length & 0.9562 & 139 & $<0.0001$ \\
Mes Bone Height & Dis Root Length & 0.9157 & 139 & $<0.0001$ \\
Dis Bone Height & Mes Root Length & 0.912 & 139 & $<0.0001$ \\
Dis Bone Height & Dis Root Length & 0.9723 & 139 & $<0.0001$ \\
Dis Bone Height & Mes Bone Height & 0.9356 & 139 & $<0.0001$ \\
Tooth Length & Mes Root Length & 0.8972 & 139 & $<0.0001$ \\
Tooth Length & Dis Root Length & 0.9125 & 139 & $<0.0001$ \\
Tooth Length & Mes Bone Height & 0.8859 & 139 & $<0.0001$ \\
Tooth Length & Dis Bone Height & 0.9035 & 139 & $<0.0001$ \\
Pulp Height & Mes Root Length & 0.8969 & 139 & $<0.0001$ \\
Pulp Height & Dis Root Length & 0.8918 & 139 & $<0.0001$ \\
Pulp Height & Mes Bone Height & 0.8769 & 139 & $<0.0001$ \\
Pulp Height & Dis Bone Height & 0.8791 & 139 & $<0.0001$ \\
Pulp Height & Tooth Length & 0.9014 & 139 & $<0.0001$ \\
MD Width & Mes Root Length & 0.1018 & 139 & 0.233 \\
MD Width & Dis Root Length & 0.1166 & 139 & 0.1715 \\
MD Width & Mes Bone Height & 0.115 & 139 & 0.1776 \\
MD Width & Dis Bone Height & 0.1362 & 139 & 0.1099 \\
MD Width & Tooth Length & 0.239 & 139 & 0.0046 \\
MD Width & Pulp Height & 0.1147 & 139 & 0.1788 \\
\hline
\end{tabular}

${ }^{1}$ Correlations were computed pairwise to take into account the missing (excluded) dimensions for some cases. 


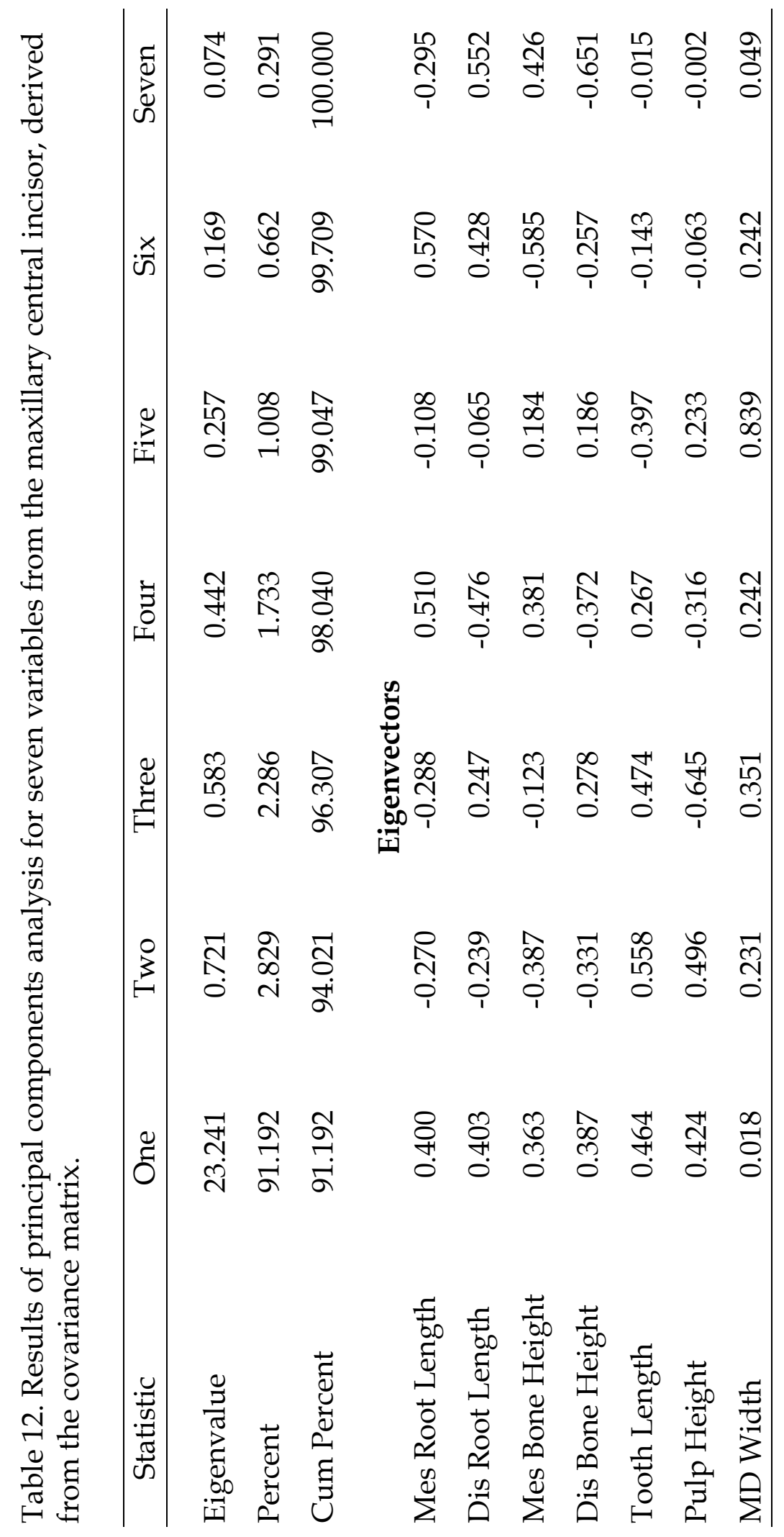


(all < 0.5), and the trivial weight of MD width on PC1 emphasizes its statistical (and biological) independence.

Only the first eigenvalue is greater than 1.0, so some would argue that none of the other components is relevant (Kaiser 1958). Still, the next few components are interpretable. PC Two is a contrast between alveolar bone heights (with negative weights) and overall tooth lengths (with positive weights). Of note, the mesial and distal root lengths both have the same signs as bone height, which probably reflects the simple fact that most of bone height, measured from the root apex, is coincident with (i.e., physically overlaps) root length.

PC Three appears to be a contrast between tooth length and pulp height, which is an association that will be explored in a later section. PC Four is a simple contrast between mesial and distal root lengths, reflecting the transverse asymmetry such that the greater deflection of the root to the distal the more unequal the mesial and distal root dimensions. PC Five is driven almost exclusively by mesiodistal crown width. PC Six and PC Seven account for less than $1 \%$ of the common variance each and are not investigated here.

Table 13 is the pairwise correlation matrix for the maxillary lateral incisor. Results are comparable as for the central incisor in that (1) correlations all are positive and very high (>0.8) among all root and alveolar bone variables but (2) mesiodistal crown size is tied comparatively weakly to these other dimensions. 
Table 13. Pairwise correlations for the maxillary lateral incisor (sexes combined). ${ }^{1}$

\begin{tabular}{llcll}
\hline Variable A & Variable B & Correlation & $\mathrm{n}$ & P-value \\
\hline Dis Root Length & Mes Root Length & 0.9162 & 137 & $<0.0001$ \\
Mes Bone Height & Mes Root Length & 0.9491 & 137 & $<0.0001$ \\
Mes Bone Height & Dis Root Length & 0.8970 & 137 & $<0.0001$ \\
Dis Bone Height & Mes Root Length & 0.8947 & 137 & $<0.0001$ \\
Dis Bone Height & Dis Root Length & 0.9573 & 137 & $<0.0001$ \\
Dis Bone Height & Mes Bone Height & 0.8933 & 137 & $<0.0001$ \\
Tooth Length & Mes Root Length & 0.9253 & 137 & $<0.0001$ \\
Tooth Length & Dis Root Length & 0.9260 & 137 & $<0.0001$ \\
Tooth Length & Mes Bone Height & 0.9066 & 137 & $<0.0001$ \\
Tooth Length & Dis Bone Height & 0.8885 & 137 & $<0.0001$ \\
Pulp Height & Mes Root Length & 0.8866 & 136 & $<0.0001$ \\
Pulp Height & Dis Root Length & 0.8919 & 136 & $<0.0001$ \\
Pulp Height & Mes Bone Height & 0.8762 & 136 & $<0.0001$ \\
Pulp Height & Dis Bone Height & 0.8514 & 136 & $<0.0001$ \\
Pulp Height & Tooth Length & 0.9206 & 136 & $<0.0001$ \\
MD Width & Mes Root Length & 0.2397 & 136 & 0.0049 \\
MD Width & Dis Root Length & 0.1915 & 136 & 0.0256 \\
MD Width & Mes Bone Height & 0.2397 & 136 & 0.0049 \\
MD Width & Dis Bone Height & 0.1809 & 136 & 0.0351 \\
MD Width & Tooth Length & 0.2814 & 136 & 0.0009 \\
MD Width & Pulp Height & 0.1689 & 136 & 0.0493 \\
\hline
\end{tabular}

${ }^{1}$ Correlations were computed pairwise to take into account the missing (excluded) dimensions for some cases. 
Statistically, the six correlation coefficients are positive and significant $(\mathrm{P}<0.05)$, but the strengths of the correlations are low and the corresponding coefficients of determination $\left(\mathrm{r}^{2}\right)$ explain less than $5 \%$ of the variation. PCA for the lateral incisor (Table 14) shows - as with the central incisor - that almost all (91.1\%) of the common variance is on the first canonical axis, which is driven by overall tooth size (i.e., all of the variables, excepting crown width, are positive and subequal in strength). PC Two is a contrast between the mesial and distal aspects of the tooth as regards root length and bone height. The largest weight for PC Two is, however, pulp height, with contrasts (negative weighting coefficients) for distal root length and distal bone height, while the coefficient for pulp height is comparatively large and positive.

PC Three has large positive weights for mesial root length and mesial bone height, and the larger negative weights are for distal root length and pulp height. Collectively, PC Three is a composite measure of mesial size. PC Four, in turn, seems to be a function of overall tooth size since it is driven primarily by (1) tooth length and (2) mesiodistal crown width, both with positive weights. The other components are ignored here since they account for so little of the variation.

It commonly is informative to use these canonical variables to test for differences among groups (e.g., Blackith and Reyment 1971). Two-way ANOVA for the first four components of the central incisor are shown in Table 15, where 


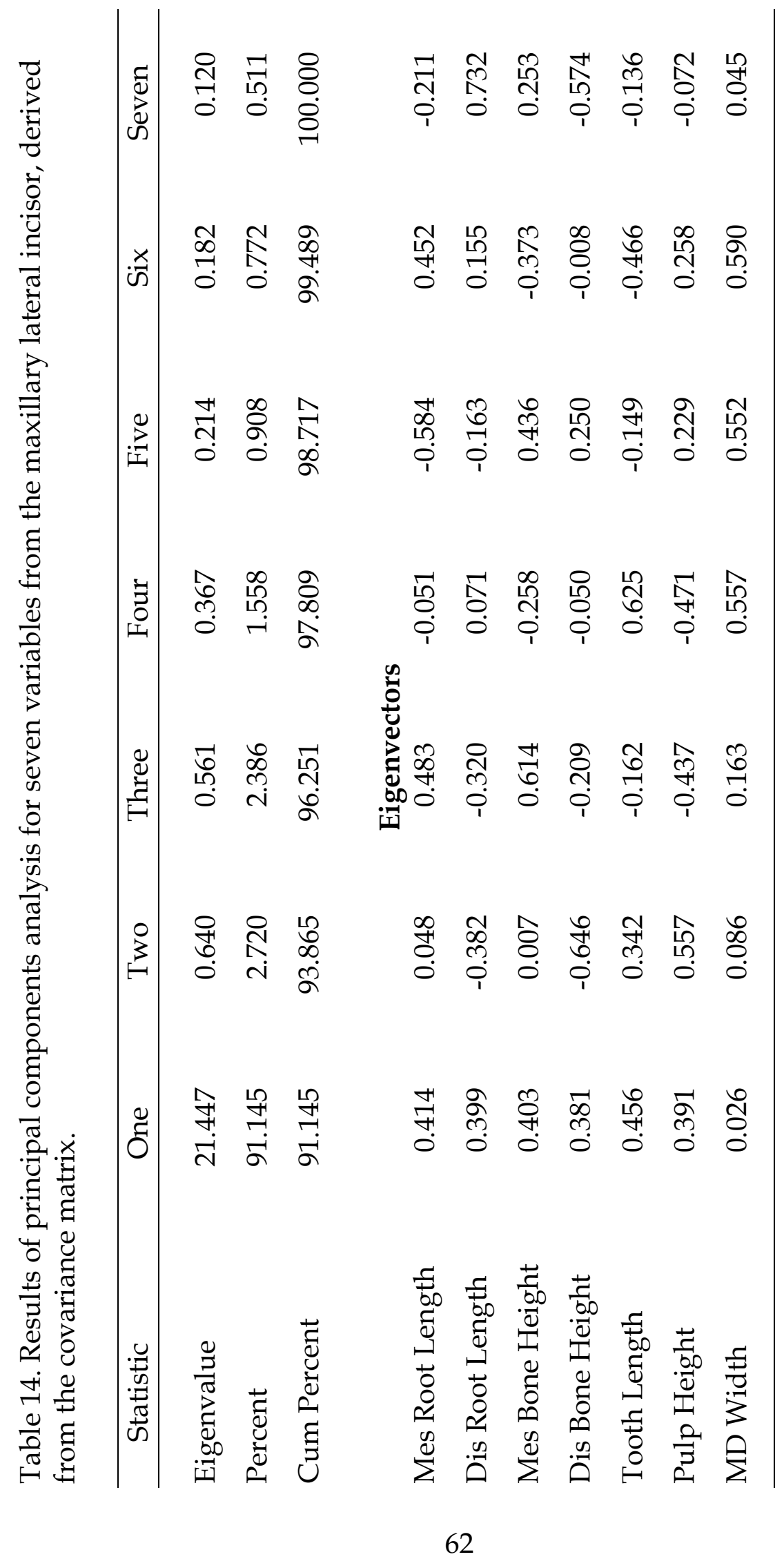


Table 15. Results of two-way analysis of variance, testing for differences by class or sex in principal components scores for the maxillary central incisor. ${ }^{1}$

\begin{tabular}{|c|c|c|c|c|c|c|}
\hline \multirow[b]{2}{*}{ Variable } & \multicolumn{2}{|c|}{ Angle Class } & \multicolumn{2}{|c|}{ Sex } & \multicolumn{2}{|c|}{ Interaction } \\
\hline & F-ratio & P-value & F-ratio & P-value & F-ratio & P-value \\
\hline PC One & 1.5623 & 0.2135 & 11.6704 & 0.0008 & 1.3159 & 0.2534 \\
\hline PC Two & 1.9609 & 0.1637 & 0.0072 & 0.9326 & 3.5266 & 0.0625 \\
\hline PC Three & 1.3447 & 0.2483 & 0.2385 & 0.6260 & 1.5433 & 0.2163 \\
\hline PC Four & 4.6119 & 0.0335 & 7.8925 & 0.0057 & 1.4556 & 0.2297 \\
\hline
\end{tabular}

${ }^{1}$ There is $1 \mathrm{df}$ associated with each of the three tests for each variable (row). 
there is a highly significant difference between the sexes for PC One (Figure 4). This is consistent with the several univariate tests showing that males have larger tooth dimensions than females. PC Two and PC Three exhibit no statistical difference as regards Angle's class or patient's sex (Figures 5 and 6). PC Four scores, which are primarily driven by mesial-distal polarities in root and bone dimensions, are significantly larger in Class II cases $(P=0.03)$ and in males compared to females $(\mathrm{P}=0.006)$. Mean component scores are graphed in Figure 7, where it is evident that most of the class and sex difference is due to the high scores in the sample of Class II males.

ANOVA results for component scores for the maxillary lateral incisor are listed in Table 16. There is a marginally significant difference between Angle's classes and between sexes (Figure 8). This first canonical axis is a function of overall tooth size, which accounts for the larger scores in males. The class difference, which is suggestive of larger lateral incisor dimensions in the Class II sample, is disproportionately dependent on the larger scores in Class II males. No significant difference occurred for PC Two (Figure 9), but there is a significant difference between Angle's classes for PC Three (Figure 10), where (as with the central incisor) the mesial-distal differences in root and bone dimensions are greater in the Class II sample. Finally, neither test is significant for PC Four (Figure 11). 


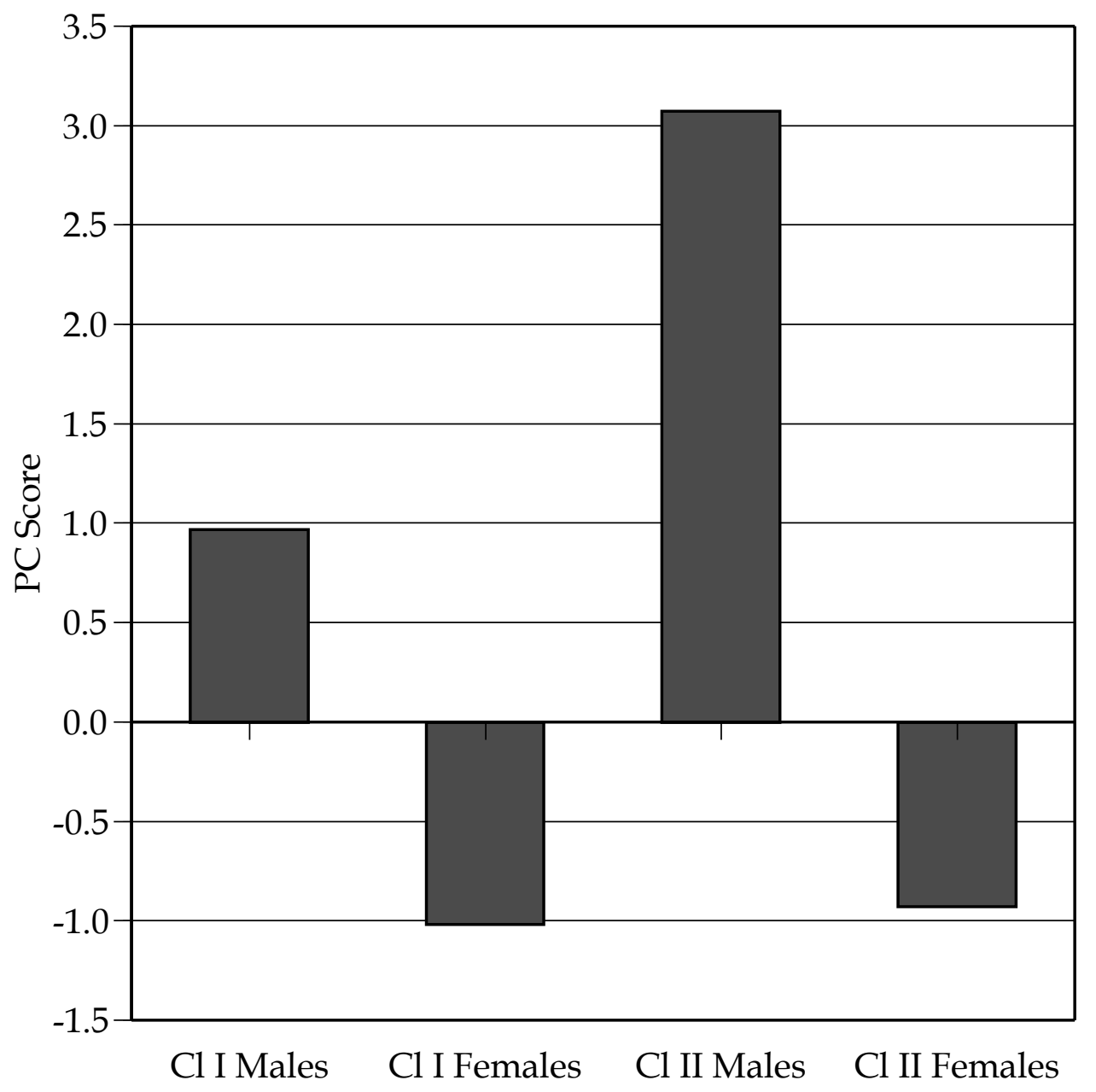

Fig. 4. Mean scores for the maxillary central incisor, PC One. 


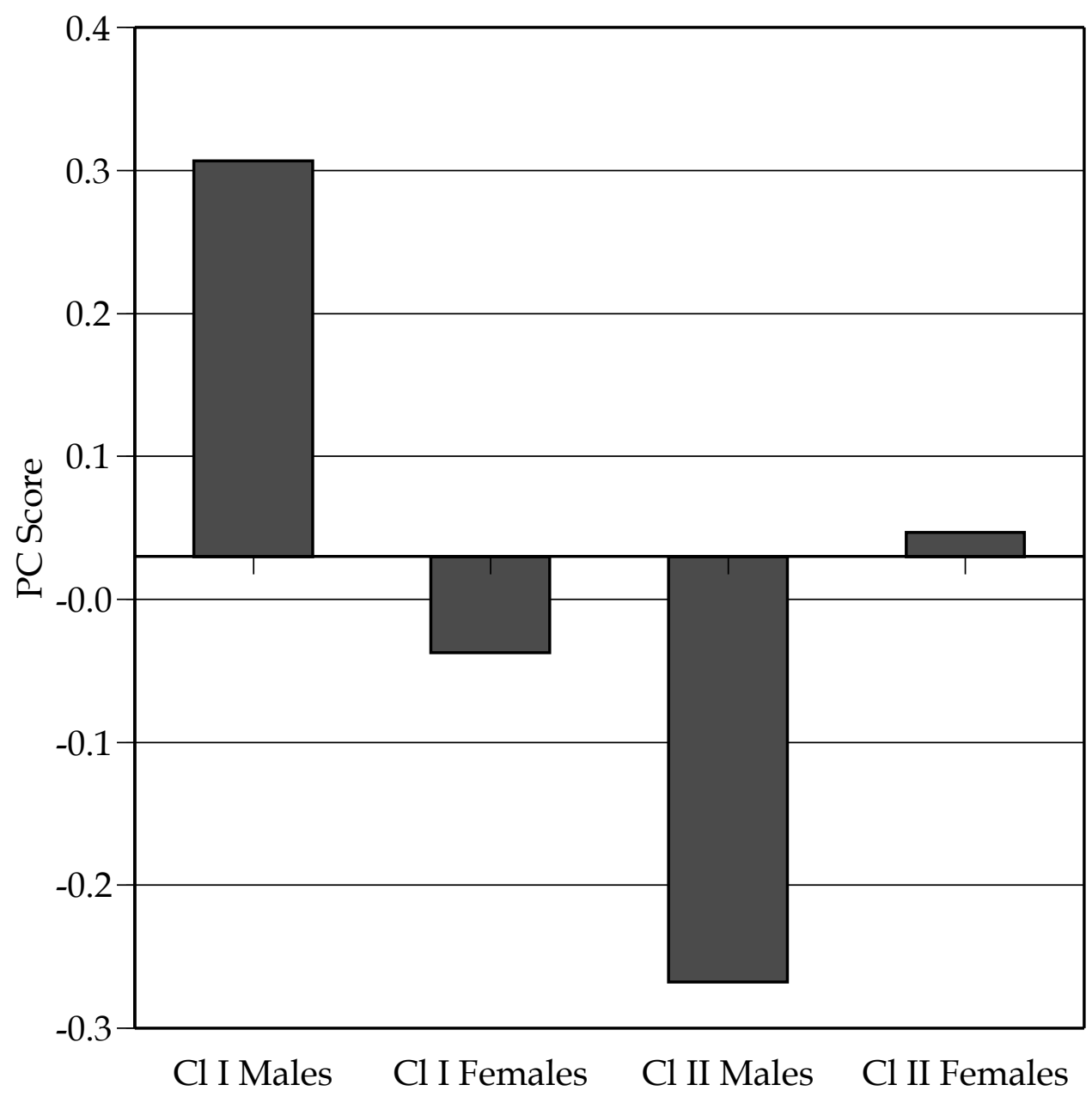

Fig. 5. Mean scores for the maxillary central incisor, PC Two. 


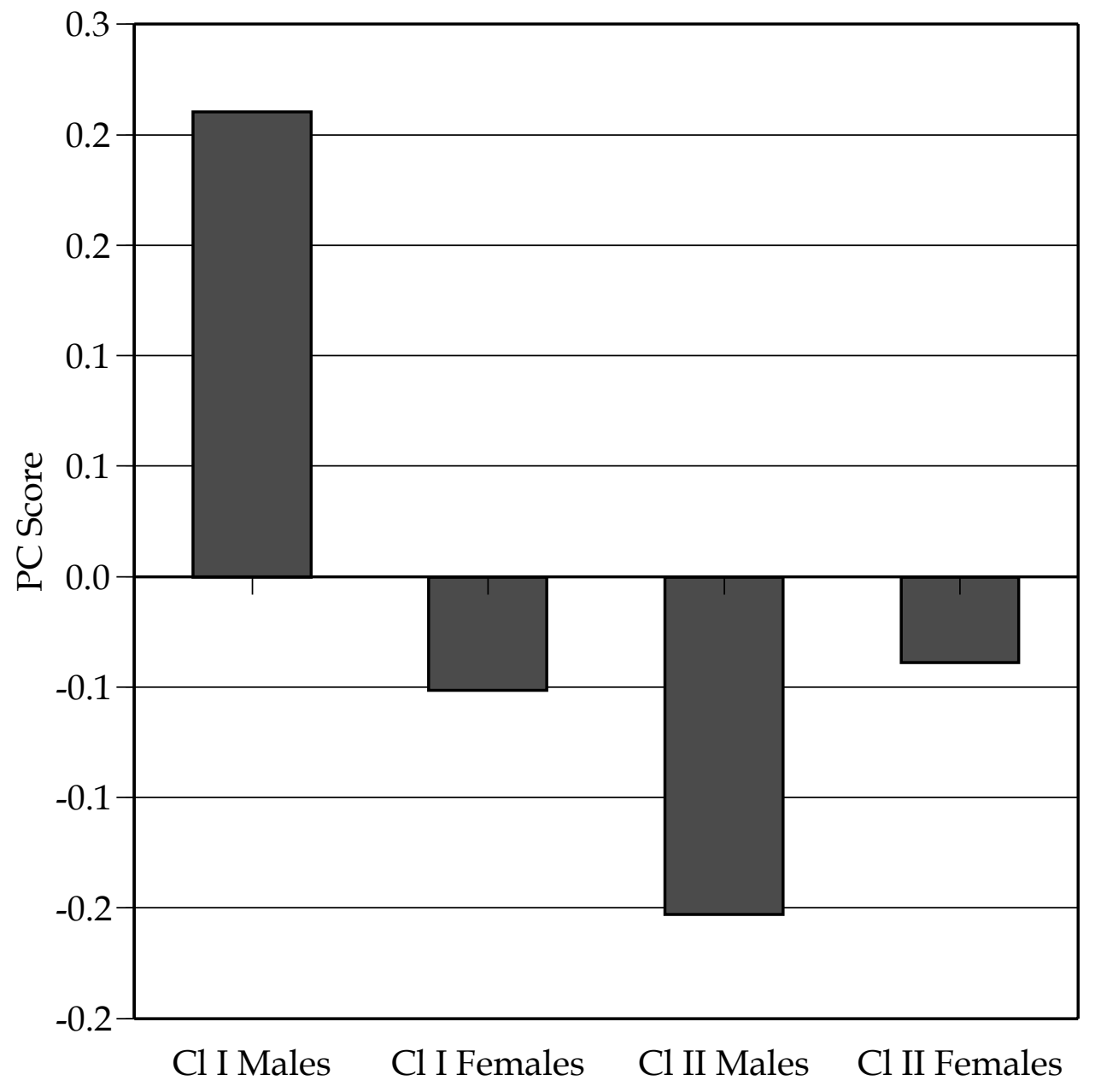

Fig. 6. Mean scores for the maxillary central incisor, PC Three. 


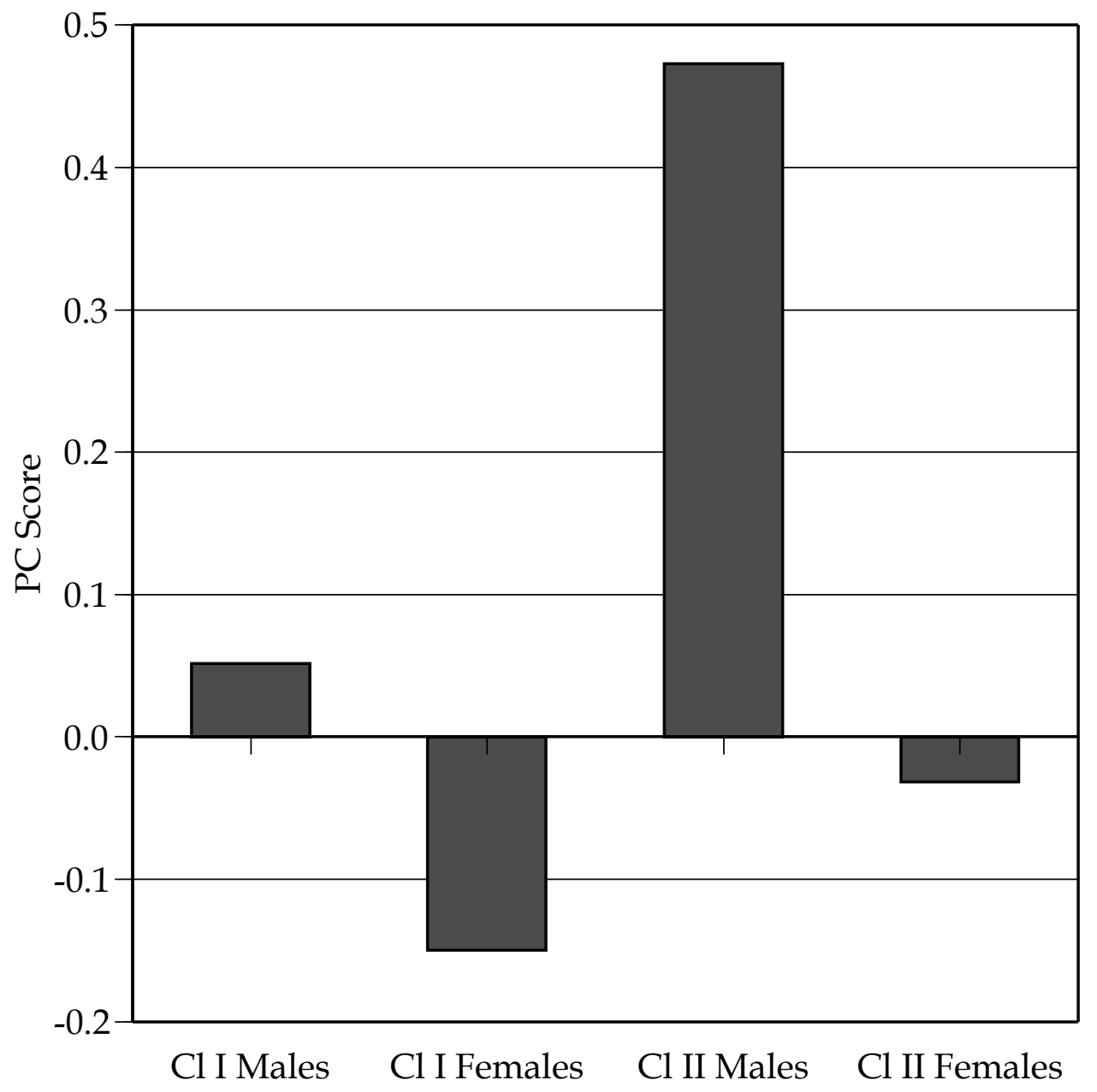

Fig. 7. Mean scores for the maxillary central incisor, PC Four. 
Table 16. Results of two-way analysis of variance, testing for differences by class or sex in principal components scores for the maxillary lateral incisor. ${ }^{1}$

\begin{tabular}{|c|c|c|c|c|c|c|}
\hline \multirow[b]{2}{*}{ Variable } & \multicolumn{2}{|c|}{ Angle Class } & \multicolumn{2}{|c|}{ Sex } & \multicolumn{2}{|c|}{ Interaction } \\
\hline & F-ratio & P-value & F-ratio & P-value & F-ratio & P-value \\
\hline PC One & 4.0629 & 0.0459 & 9.3775 & 0.0027 & 0.6889 & 0.4080 \\
\hline PC Two & 0.0817 & 0.7755 & 0.0651 & 0.7990 & 0.0919 & 0.7623 \\
\hline PC Three & 5.1724 & 0.0246 & 2.0124 & 0.1584 & 0.0578 & 0.8104 \\
\hline PC Four & 2.1735 & 0.1428 & 0.1744 & 0.6769 & 2.5174 & 0.1150 \\
\hline
\end{tabular}

${ }^{1}$ There is $1 \mathrm{df}$ associated with each of the three tests for each variable (row). 


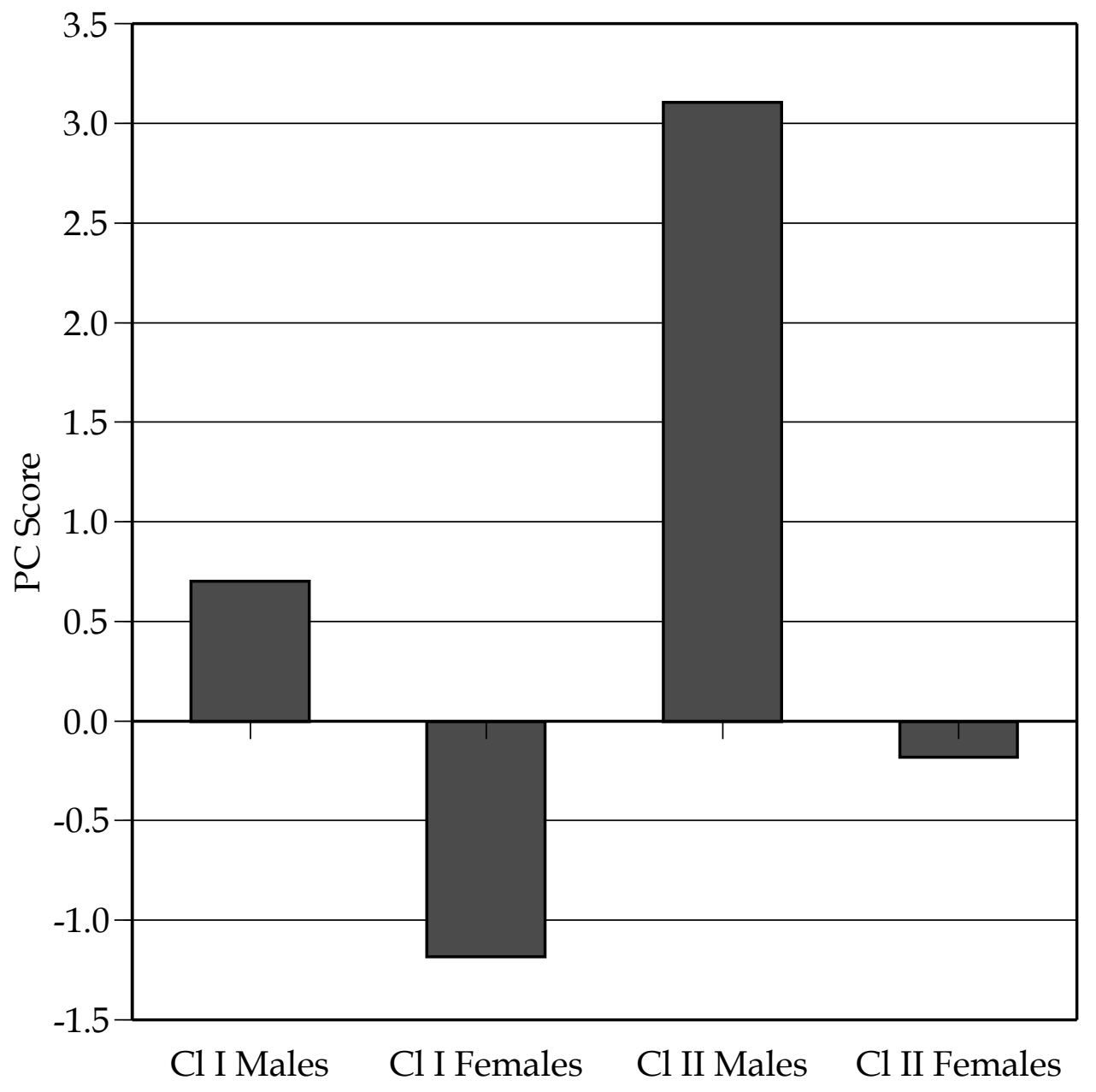

Fig. 8. Mean scores for the maxillary lateral incisor, PC One. 


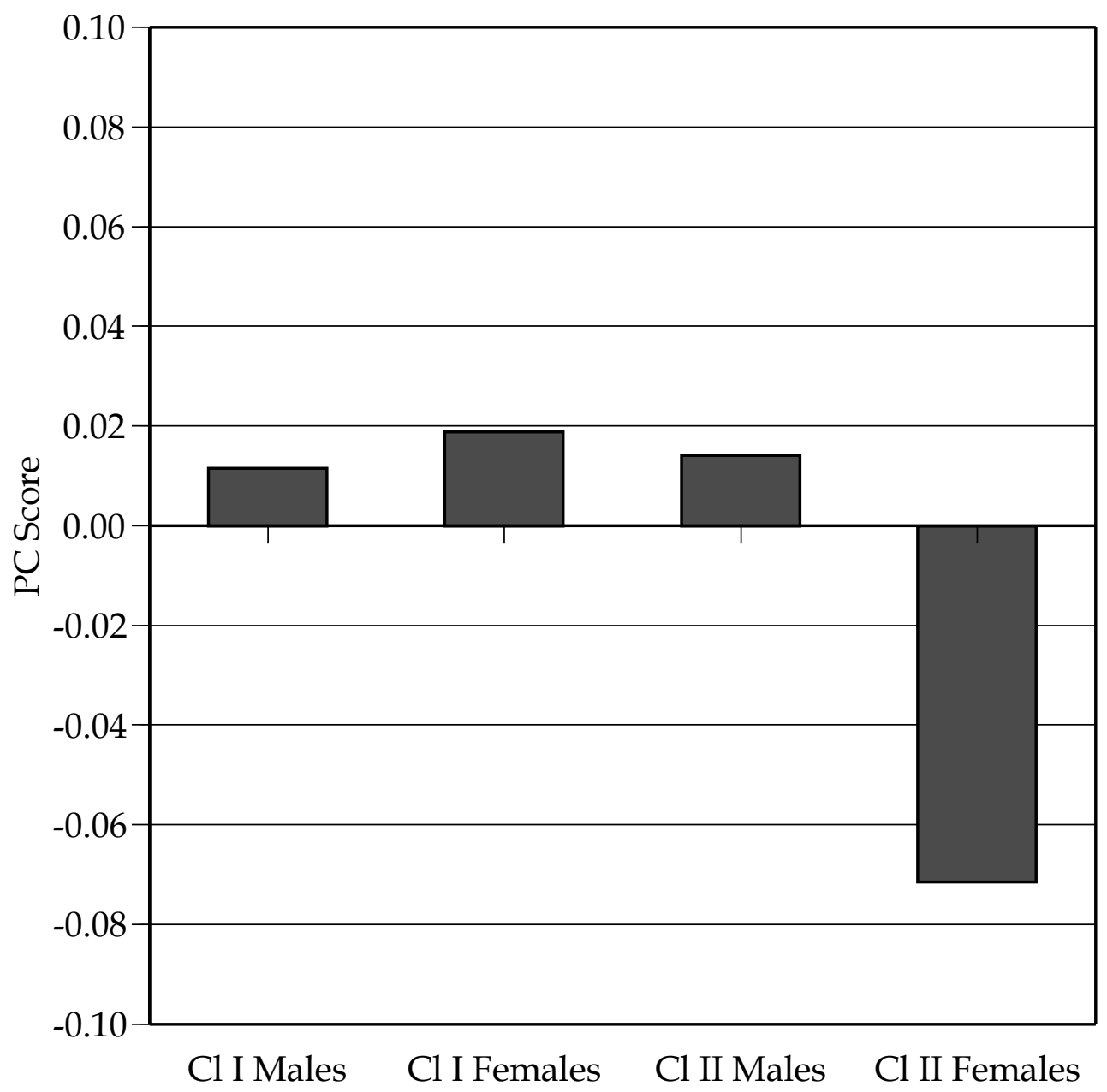

Fig. 9. Mean scores for the maxillary lateral incisor, PC Two. 


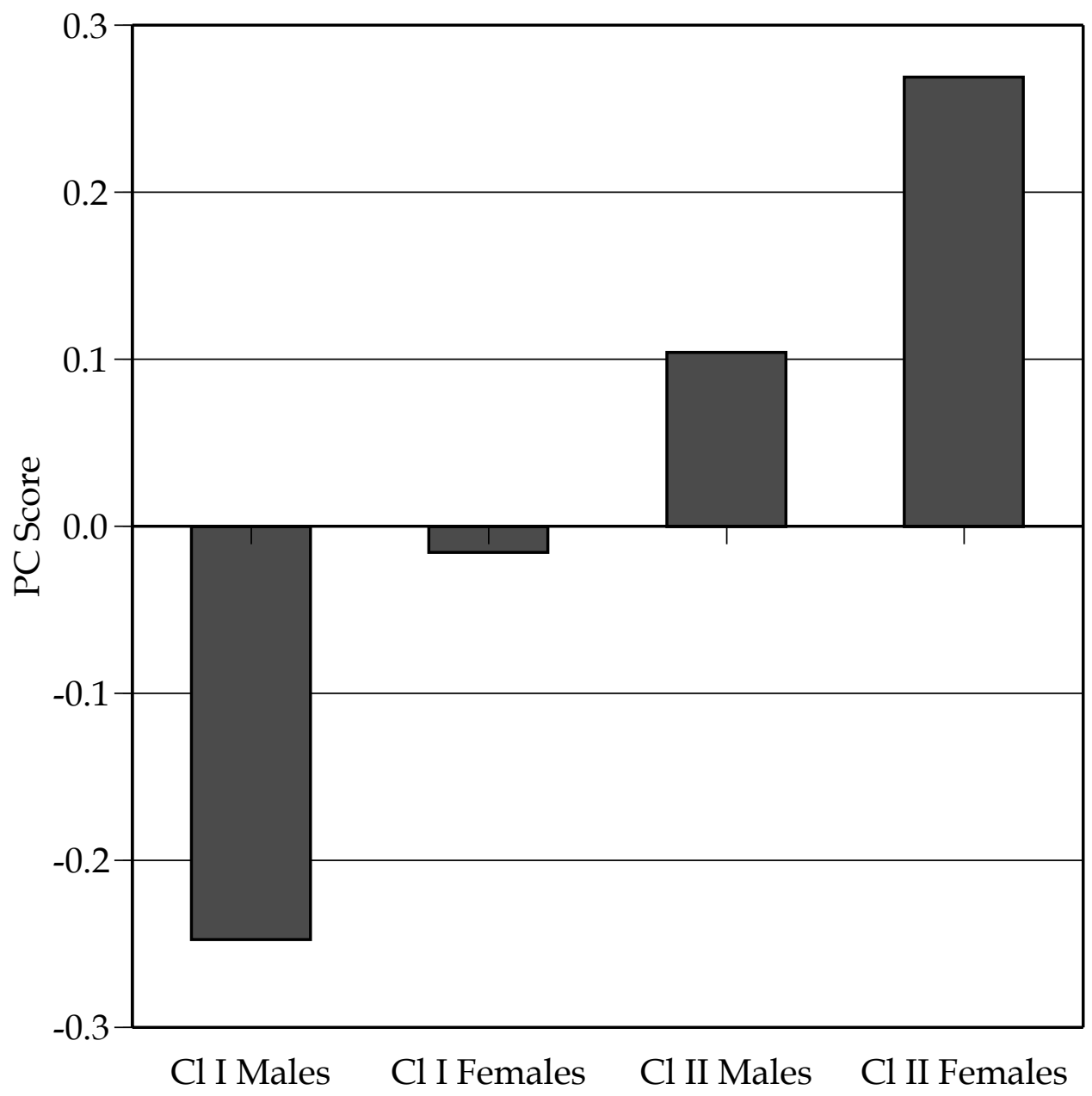

Fig. 10. Mean scores for the maxillary lateral incisor, PC Three. 


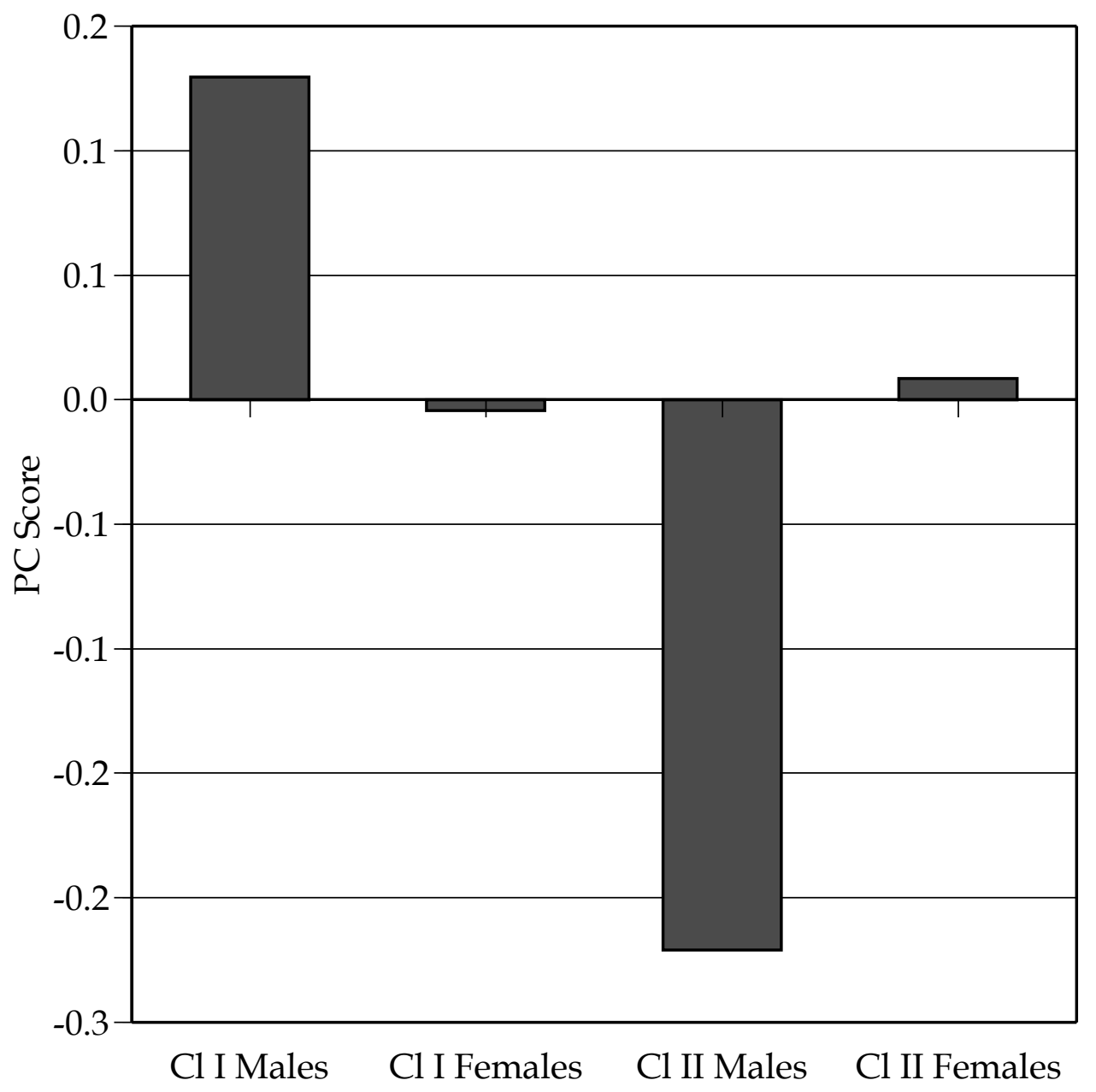

Fig. 11. Mean scores for the maxillary lateral incisor, PC Four. 


\section{Crown Heights and Root Lengths}

Height of the dentinoenamel junction undulates around the incisor, being higher (more coronal) on the medial and lateral aspects and lower (more apical) on the lingual and facial aspects (e.g., Zeisz and Nuckolls 1949). Consequently, there is no invariant crown or root length. Root length was calculated in this study by identifying the medial and lateral CEJ from the facial view of each incisor's periapical radiograph. Then, as described in Methods, the straight-line apex-CEJ distance was measured on the tooth's medial and lateral margin. Root length was defined as the arithmetic mean of these two distances. Crown height then was calculated as tooth length (apex to incisal edge; Figure 1) minus root length.

These root lengths and crown heights are listed in Table 17, along with analysis of variance tests for sexual dimorphism. Results show that root length is longer on the maxillary central incisor than the lateral incisor. By paired t-test this difference, averaging $0.3 \mathrm{~mm}$, is statistically significant $(\mathrm{t}=2.38 ; \mathrm{P}=0.0187)$. In contrast, the root of the mandibular lateral incisor is longer than the central incisor, by an average of $2.1 \mathrm{~mm}$, which is highly significant statistically $(\mathrm{t}=26.2$; $\mathrm{P}<<0.0001)$. This "reversal," where the mandibular lateral incisor is larger than the central incisor, is the only instance in the human dentition where the distal tooth in the tooth type is bigger (and less variable) than the mesial element within a morphogenetic field (Dahlberg 1945). The results listed in Table 17 also 
Table 17. Descriptive statistics and tests for sexual dimorphism of root and crown heights. ${ }^{1}$

\begin{tabular}{|c|c|c|c|c|c|c|c|}
\hline \multirow[b]{2}{*}{ Variable } & \multicolumn{2}{|c|}{ Males } & \multicolumn{2}{|c|}{ Females } & \multirow[b]{2}{*}{$\%$} & \multicolumn{2}{|c|}{ ANOVA } \\
\hline & $\mathrm{n}$ & $\bar{x}$ & $\mathrm{n}$ & $\bar{x}$ & & F-ratio & P-value \\
\hline \multicolumn{8}{|c|}{ Root Lengths } \\
\hline U1 Root & 51 & 18.02 & 88 & 16.95 & 6.28 & 10.17 & 0.0018 \\
\hline U2 Root & 50 & 17.64 & 87 & 16.65 & 5.95 & 9.01 & 0.0032 \\
\hline L1 Root & 51 & 14.59 & 88 & 13.58 & 7.38 & 15.21 & 0.0001 \\
\hline L2 Root & 51 & 16.60 & 88 & 15.80 & 5.03 & 7.95 & 0.0055 \\
\hline \multicolumn{8}{|c|}{ Crown Heights } \\
\hline U1 Crown & 51 & 8.38 & 88 & 8.23 & 1.85 & 0.90 & 0.3454 \\
\hline U2 Crown & 50 & 7.38 & 87 & 7.12 & 3.64 & 4.02 & 0.0468 \\
\hline L1 Crown & 51 & 7.92 & 88 & 7.98 & -0.75 & 0.12 & 0.7298 \\
\hline L2 Crown & 51 & 7.11 & 88 & 7.22 & -1.54 & 0.58 & 0.4473 \\
\hline
\end{tabular}

$1 \%$ is percent sexual dimorphism, calculated as (M-F)/F times 100. 
show that root lengths are sexually dimorphic, with males averaging 5 to $7 \%$ longer roots than females.

Statistics for incisor crown heights also are listed in Table 17. The maxillary central incisor is, on average, $1.1 \mathrm{~mm}$ taller than the lateral incisor $(\mathrm{t}=$ 15.0; $\mathrm{P}<0.0001)$, and both of these tooth types are taller in males than females, but the differences are not statistically significant for the maxillary central incisor and only marginally significant for the lateral incisor. There seems to be some correspondence between the lateral incisor being significantly taller (the only one of the four incisor types to be significant) and the appreciably greater sexual dimorphism for this tooth type. That is, sexual dimorphism is near-zero for the other three incisor types, but $4 \%$ for the upper lateral incisor. Given the notoriously high prevalence of small and pegged U2 in Caucasoid females (Ballard 1944; Crosby 1972), these data can be interpreted as preferential reduction in UI2 crown heights in females.

Mandibular incisor crown heights are significantly taller for the central than the lateral elements $(\mathrm{t}=14.6 ; \mathrm{P}<0.0001)$, a mean difference of $0.8 \mathrm{~mm}$, which runs counter to the assumed field reversal noted above for the root lengths. Of note, too, both lower incisors are trivially taller in females than males, as reflected in the negative measures of sexual dimorphism. 


\section{Crown-Root Ratios}

Crown-to-root ratios were calculated individually as anatomic crown height divided by root length $(\mathrm{CH} / \mathrm{RL})$. These ratios for the four incisor tooth types (Table 18) are on the order of $50 \%$, showing that root length is about twice crown height. Crown height is not as variable as root length, so with this system $(\mathrm{CH} / \mathrm{RL})$, the ratio is essentially a measure of root length, and the larger the ratio the shorter the root vis-à-vis the crown. The ANOVA tests for sexual dimorphism suggest that the crown-root ratios are the same for the two maxillary incisors, though the $\mathrm{P}$-value for the maxillary central incisor $(\mathrm{P}=0.07)$ is suggestive. Looking just at the raw numbers, root length is $49 \%$ of crown height of maxillary central incisors in girls but very slightly less, $47 \%$, in boys. Analysis (Table 17) shows that maxillary central incisor crown heights do not differ statistically between the sexes, so the difference in the ratio is attributable predominantly to an (insignificantly) shorter root-both absolutely and relative to crown height - in girls. Both of the mandibular incisor tooth types have significantly higher crown-root ratios in girls than boys (Table 18). In other words, roots are disproportionately short relative to crown height in girls. This may well reflect the greater masticatory forces of males that have to be absorbed by the root surfaces.

Table 18 lists the statistics of dividing crown heights by root lengths. One obvious feature is that the crown heights are on the order of $40-50 \%$ of root 
Table 18. Descriptive statistics and tests for sexual dimorphism of crown-root ratios. ${ }^{1}$

\begin{tabular}{|c|c|c|c|c|c|c|c|}
\hline \multirow[b]{2}{*}{ Variable } & \multicolumn{2}{|c|}{ Males } & \multicolumn{2}{|c|}{ Females } & \multirow[b]{2}{*}{$\%$} & \multicolumn{2}{|c|}{ ANOVA } \\
\hline & $\mathrm{n}$ & $\bar{x}$ & $\mathrm{n}$ & $\bar{x}$ & & F-ratio & P-value \\
\hline $\mathrm{U} 1 \mathrm{C} / \mathrm{R}$ & 51 & 0.469 & 88 & 0.491 & -4.57 & 3.39 & 0.0677 \\
\hline $\mathrm{U} 2 \mathrm{C} / \mathrm{R}$ & 50 & 0.422 & 87 & 0.432 & -2.31 & 0.93 & 0.3365 \\
\hline L1 C/R & 51 & 0.548 & 88 & 0.591 & -7.29 & 9.76 & 0.0022 \\
\hline $\mathrm{L} 2 \mathrm{C} / \mathrm{R}$ & 51 & 0.432 & 88 & 0.459 & -5.83 & 8.03 & 0.0053 \\
\hline
\end{tabular}

${ }^{1}$ Crown-root ratio is crown height divided by root length; \% is percent sexual dimorphism, calculated as (M-F)/F times 100. 
lengths. Another is that crown-root ratios are larger in females than males. The ratio is significantly larger for L1 and L2; it is marginally different for U1 ( $\mathrm{P}=$ $0.06)$; and nonsignificant for $\mathrm{U} 2(\mathrm{P}=0.34)$. There is the interesting relationship, then, that crown heights tend to be slightly taller in males, but the roots are disproportionately long in males, so the crown-root ratios tend to be greater in females (Table 18).

\section{Crown and Root Lengths}

Overall tooth length (root apex to incisal edge) was measured on each tooth, as was the root length proper (root apex to the mesial and distal CEJ average). Anatomical crown height was figured as the difference (tooth length minus root length). These dimensions are listed in Table 17, with the two sexes separated because of the well-known sex differences (e.g., Garn et al. 1967; Garn et al. 1978a,b,c). Indeed, the ANOVA tests disclose highly significant differences for all four root lengths, where percent sexual dimorphism is on the order of 5 to $7 \%$. Percentagewise, these sex differences handily exceed those for crown size, so they may be useful for sex-assignment in forensic settings (Harris and Couch 2006). Anatomic crown heights, on the other hand, are not discernibly different between the two sexes, except for a marginally-significant difference $(P=0.047)$ for the maxillary lateral incisor, where males have a $4 \%$ longer mean crown height. 


\section{Crown Height-Width Ratios}

Proportionality of the mesiodistal widths of the incisors are an important esthetic consideration (Neff 1949; Lundström 1954), and it also affects the orthodontist's ability to get the teeth to couple properly (Bolton 1958, 1962). Ratios of the maximum crown mesiodistal widths were calculated in each arch by dividing width of the lateral incisor by that of the central incisor (Table 19).

This ratio is less than one in the upper arch (because U1 is broader than U2) but greater than one in the lower arch, where L2 is the broader tooth type. Neither ratio suggests a sex difference, so, while boys have larger tooth crowns than girls, the crown proportionalities (shape) are the same. Combining sexes (since they are the same for these variables), the maxillary lateral to central ratio is $0.771(\mathrm{sd}=0.055 ; \mathrm{sem}=0.005 ; \mathrm{n}=136)$ and the mandibular ratio averages 1.105 $(\mathrm{sd}=0.054 ; \mathrm{sem}=0.005 ; \mathrm{n}=139)$.

\section{$\underline{\text { Alveolar Bone Heights }}$}

Bone heights were calculated as the difference between (1) the distance from the root apex to the CEJ and (2) the distance from the root apex to the crestal bone's margin adjacent to the tooth. This was done separately on each incisor's medial and lateral aspect. Specifically, bone height was subtracted from root length, so a positive difference (as occurs with all variables) indicates that the crestal bone stops short of (apical to) the tooth's CEJ. 
Table 19. Ratios of lateral to central incisor widths, by sex, in the two arcades.

\begin{tabular}{|c|c|c|c|c|c|c|c|c|}
\hline & \multicolumn{3}{|c|}{ Males } & \multicolumn{3}{|c|}{ Females } & \multicolumn{2}{|c|}{ ANOVA } \\
\hline & $\mathrm{n}$ & $\bar{x}$ & se & $\mathrm{n}$ & $\bar{x}$ & se & F-ratio & $P$ P-value \\
\hline Mx U2-U1 & 49 & 0.766 & 0.008 & 87 & 0.774 & 0.006 & 0.70 & 0.4052 \\
\hline Md L2-L1 & 51 & 1.114 & 0.008 & 88 & 1.100 & 0.006 & 2.18 & 0.1424 \\
\hline
\end{tabular}


Descriptive statistics are listed in Table 20, and inspection shows that crestal bone is about a millimeter apical to the tooth's cementoenamel junction. On the other hand, there is an obvious arcade difference: All of the means in the maxilla exceed a millimeter, while most of the means in the mandible are less than a millimeter. In other words, bone heights are closer to the CEJ in the mandibular teeth in these healthy adolescents, at least as viewed on these periapical radiographs. Testing the four incisor tooth types for a difference between boys and girls (Table 20) shows that there is no statistical difference. As an aside, we also tested for an age effect since researchers have documented a proliferation of alveolar crestal bone after the completion of tooth emergence (e.g., Carlson 1944). That is, when a tooth first emerges into occlusion, crestal bone height is lower (more apical) to the CEJ than observed some years later. We tested this using analysis of covariance, with crestal bone height as the dependent variable, sex as the fixed treatment effect, and age at examination as the covariate. In none of the eight tests was "age" at all predictive. The incisors all emerge during what van der Linden and Duterloo (1976) term the first transition, between 6 and 8 years of age. Perhaps our cross-sectional examination of adolescents during the teenage years is too long after the event to pick up this maturation of the bone height relative to the CEJ.

Table 21 lists the results of paired t-tests that assess whether crestal bone heights are equivalent on the medial and lateral aspects of a tooth. Recall that 


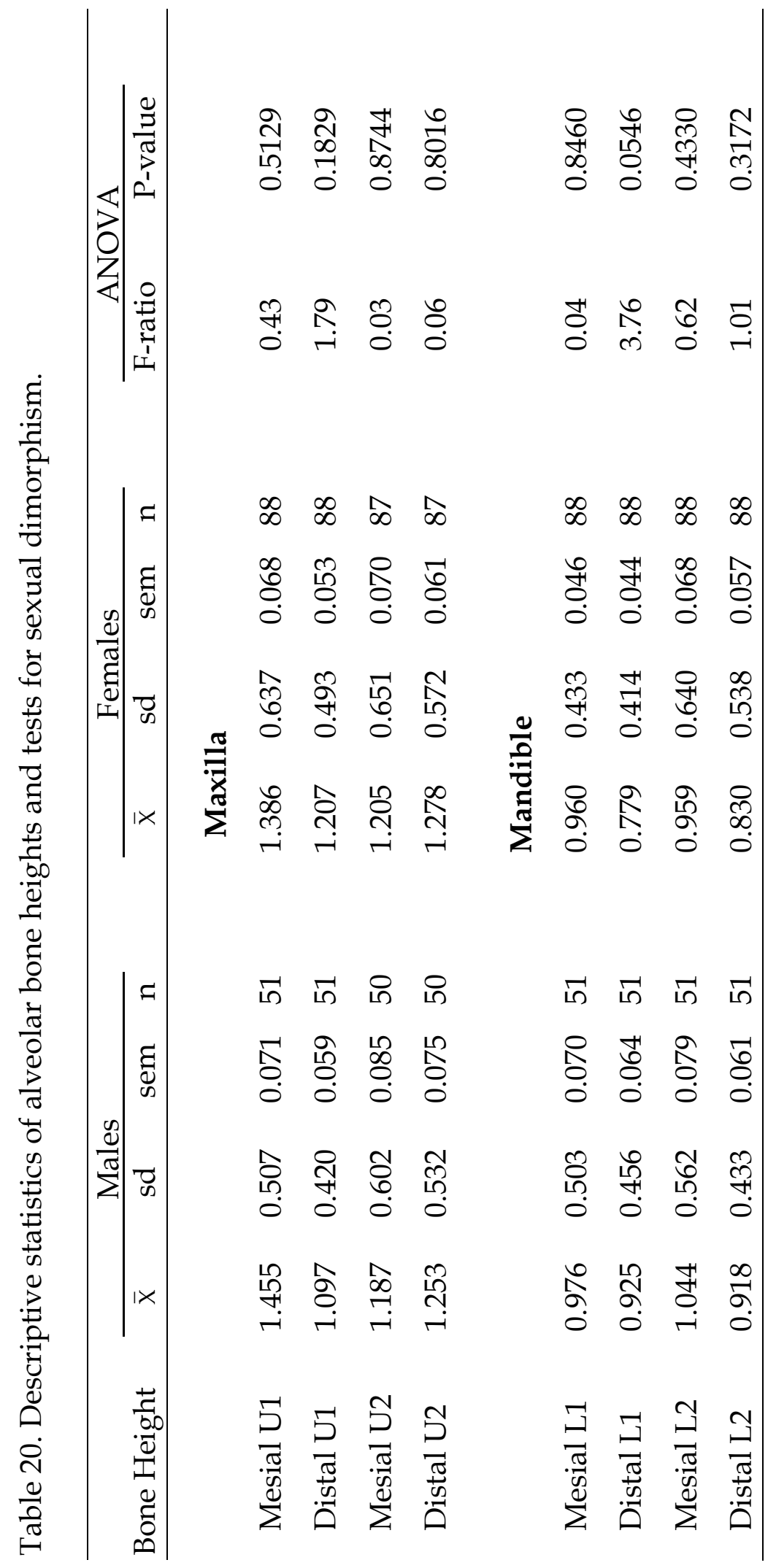


Table 21. Paired comparisons of bone heights between sides of each tooth, medial and lateral. ${ }^{1}$

\begin{tabular}{ccccccr}
\hline Variable & $\begin{array}{c}\text { Medial } \\
\overline{\mathrm{x}}\end{array}$ & $\begin{array}{c}\text { Lateral } \\
\overline{\mathrm{x}}\end{array}$ & $\begin{array}{c}\text { Mean } \\
\text { Difference }\end{array}$ & $\begin{array}{c}\text { Paired } \\
\text { t-test }\end{array}$ & $\mathrm{df}$ & P-value \\
\hline $\mathrm{U} 1$ & 1.41 & 1.17 & -0.25 & -4.83 & 138 & $<0.0001$ \\
$\mathrm{U} 2$ & 1.20 & 1.27 & 0.07 & 1.09 & 136 & 0.2767 \\
$\mathrm{~L} 1$ & 0.97 & 0.83 & -0.13 & -2.57 & 138 & 0.0112 \\
L2 & 0.99 & 0.86 & -0.13 & -2.23 & 138 & 0.0271 \\
\hline
\end{tabular}

${ }^{1}$ Sexes are combined since prior tests disclosed no sexual dimorphism. 
these bone heights are measured relative to the tooth's CEJ, which undulates around the incisor's periphery and is more coronal on the medial and lateral surfaces. These distances should not be confused with periodontal probing depths that can be reflective of pathological, degradative processes.

Three of the four tests are statistically significant (Table 21), and, in each case, the dimension is larger on the tooth's medial aspect. Again, these dimensions are the distances from the margin of the crestal bone coronally to the $\mathrm{CEJ}$, so the tests disclose that the bone is lower (more apical) on the incisor's medial than its lateral aspect. The mean differences calculated on an individual basis (not the difference of means) are small, only about 0.1 to $0.2 \mathrm{~mm}$, but their considerable statistical difference shows that what aspect of a tooth is being measured when, as here, the precision of the method is considerable.

\section{$\underline{\text { Pulp Dimensions }}$}

Largely for completeness, we also analyzed two pulp dimensions. One is simply pulp height, measured from the incisor's root apex linearly to landmark E (incisal limit of the pulp chamber in the mesiodistal third of the crown). The second variable here is tooth length minus pulp height (Figure 1), which is the incisal portion of the crown coronal to the pulp chamber.

ANOVA tests for sexual dimorphism are listed in Table 22. Percent dimorphism is on the order of 5 to $7 \%$ for pulp height, and all four tooth types 


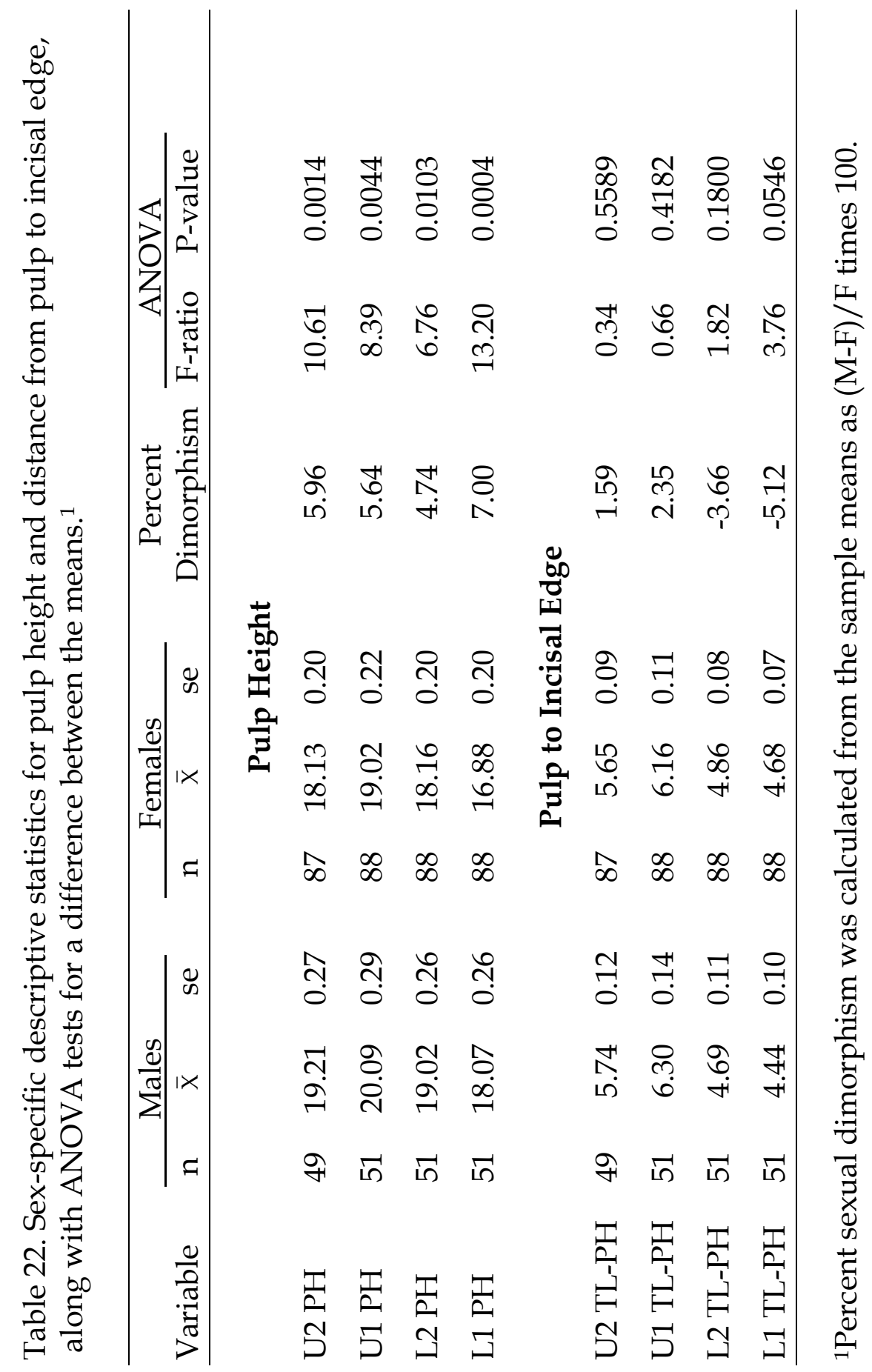


show that males have significantly longer pulp heights than females. This is predicable since pulp height geometrically is largely overlapping of root length, and, as seen above, root lengths are themselves significantly dimorphic.

Also, a mixed model ANOVA was used to compare pulp heights between U1 and U2 in the maxilla, which accounts for the sexual dimorphism. Tooth type differences (U1 vs. U2) have significantly different pulp heights, which probably reflect their differences in root lengths. Pulp height is significantly greater in the central incisor $(\bar{x}=19.41 \mathrm{~mm})$ compared to the lateral incisor $(\bar{x}=$ $18.52 \mathrm{~mm}$ ), yielding a tooth-type F-ratio of 34.16 ( 1 and $134 \mathrm{df})$ with $\mathrm{P}<0.0001$.

This L1-L2 comparison also is highly significant in the mandible. Here, though, L1 pulp height is shorter $(\bar{x}=17.32 \mathrm{~mm})$ than $\mathrm{I} 2(\overline{\mathrm{x}}=18.48 \mathrm{~mm})$, with an associated F-ratio of $113.92(\mathrm{df}=1$ and 137) and $\mathrm{P}<0.0001$.

Tooth length minus pulp height is labeled "Pulp to Incisal Edge" in Table 22 , and none of these four tests is significantly different between sexes. Indeed, there is negative dimorphism (females larger than males) for the two mandibular incisor types.

On the other hand, comparing between incisor tooth types within each jaw shows that this distance (TL - $\mathrm{PH})$ is significantly larger in the maxillary central incisor $(\bar{x}=6.21 \mathrm{~mm})$ than the upper lateral incisor $(\bar{x}=5.68 \mathrm{~mm})$. By paired $t$-test, $t=5.33$ with $P<0.0001$. In contrast, in the mandible, this distance (TL-PH) is greater in the lateral $(\overline{\mathrm{x}}=4.80 \mathrm{~mm})$ rather than the central incisor 
$(\bar{x}=4.59 \mathrm{~mm})$, with the difference being highly significant $(\mathrm{P}<0.0001)$ by paired t-test $(\mathrm{t}=3.14 ; \mathrm{df}=138)$. 


\section{CHAPTER V}

\section{DISCUSSION}

Our intention in this study was to better understand incisor crown and root dimensions of adolescents prior to orthodontic correction. Having established contemporary metric crown and root standards in our cases at pretreatment, we can compare to posttreatment data and better understand the nature, causes, and severity of external apical root resorption.

The methodology of this study is an improvement over that of G. V. Black's (1897) commonly-cited statistics based on extracted teeth. In contrast, in the present study sex, age, and race have been controlled for, thus eliminating questions of whether combining these groups in the sample skews the results. We also do not know if Black's teeth had been pathologically altered. Black's statistics were carried out to tenths of millimeters and are listed as follows: maxillary central incisor tooth length $(22.5 \mathrm{~mm})$, crown height $(10.0 \mathrm{~mm})$, root length $(12.0 \mathrm{~mm})$, crown width $(9.0 \mathrm{~mm})$; maxillary lateral incisor tooth length (22.0 mm), crown height $(8.8 \mathrm{~mm})$, root length $(13.0 \mathrm{~mm})$, crown width $(6.4 \mathrm{~mm})$; mandibular central incisor tooth length $(20.7 \mathrm{~mm})$, crown height $(8.8 \mathrm{~mm})$, root length $(11.8 \mathrm{~mm})$, crown width $(5.4 \mathrm{~mm})$; and mandibular lateral incisor tooth length $(21.1 \mathrm{~mm})$, crown height $(9.6 \mathrm{~mm})$, root length $(12.7 \mathrm{~mm})$ and crown width $(5.9 \mathrm{~mm})$. Just comparing crown widths, the present study's means for the 
maxillary central incisor were $8.9 \mathrm{~mm}$ (males) and $8.7 \mathrm{~mm}$ (females) where Black's averaged $9.0 \mathrm{~mm}$. The other three incisor types in the present study did not stray much from Black's averages (Figure 12).

When comparing tooth lengths in the present study, the maxillary central incisor averaged $26.4 \mathrm{~mm}$ (males) and $22.5 \mathrm{~mm}$ (females), while Black's averaged $22.5 \mathrm{~mm}$. Here, the male means were much larger Black's. Also, our male means for maxillary lateral tooth length $(25.1 \mathrm{~mm})$ was considerably larger than Black's (22.0 mm). Our female means for tooth length did not differ as much when compared to Black's corresponding measurements (Figure 13).

Harris and Burris (2003) made comparisons to Black's crown size data and found their contemporary data on American whites differed from Black's. If we just look at the maxillary central incisor crown widths, the males' mean of 8.6 $\mathrm{mm}$ and the females' mean of $8.4 \mathrm{~mm}$ are significantly different from Black's mean of $9.0 \mathrm{~mm}$. Also a $3.1 \%$ sexual dimorphism was observed in the data presented by Harris and Burris. Our results of $8.9 \mathrm{~mm}$ (males) and $8.7 \mathrm{~mm}$ (females) lie between the results of the two previous studies. For the mandibular central incisor widths, the male's mean of $5.3 \mathrm{~mm}$ and female's mean of $5.3 \mathrm{~mm}$ are not significantly different from Black's mean of $5.4 \mathrm{~mm}$. Our results of 5.5 mm (males) and $5.4 \mathrm{~mm}$ (females) are slightly larger than Harris and Burris'. Our present study was comparable to Harris and Burris' technique for cast measurement, and this study also determined root length, crown height and 


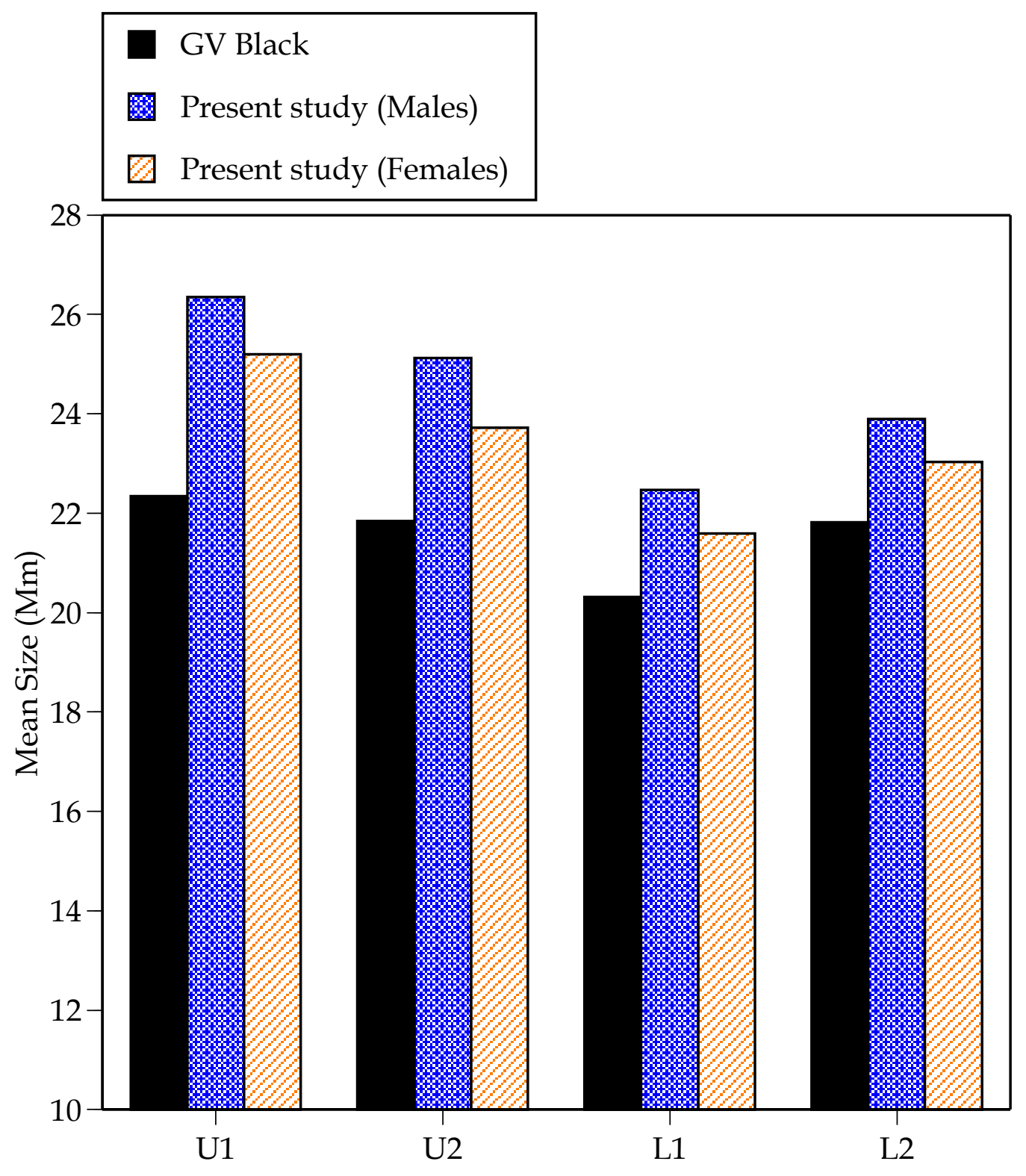

Fig. 12. Comparisons of overall tooth lengths between G. V. Black's data and the present study (where data are presented by sex). 


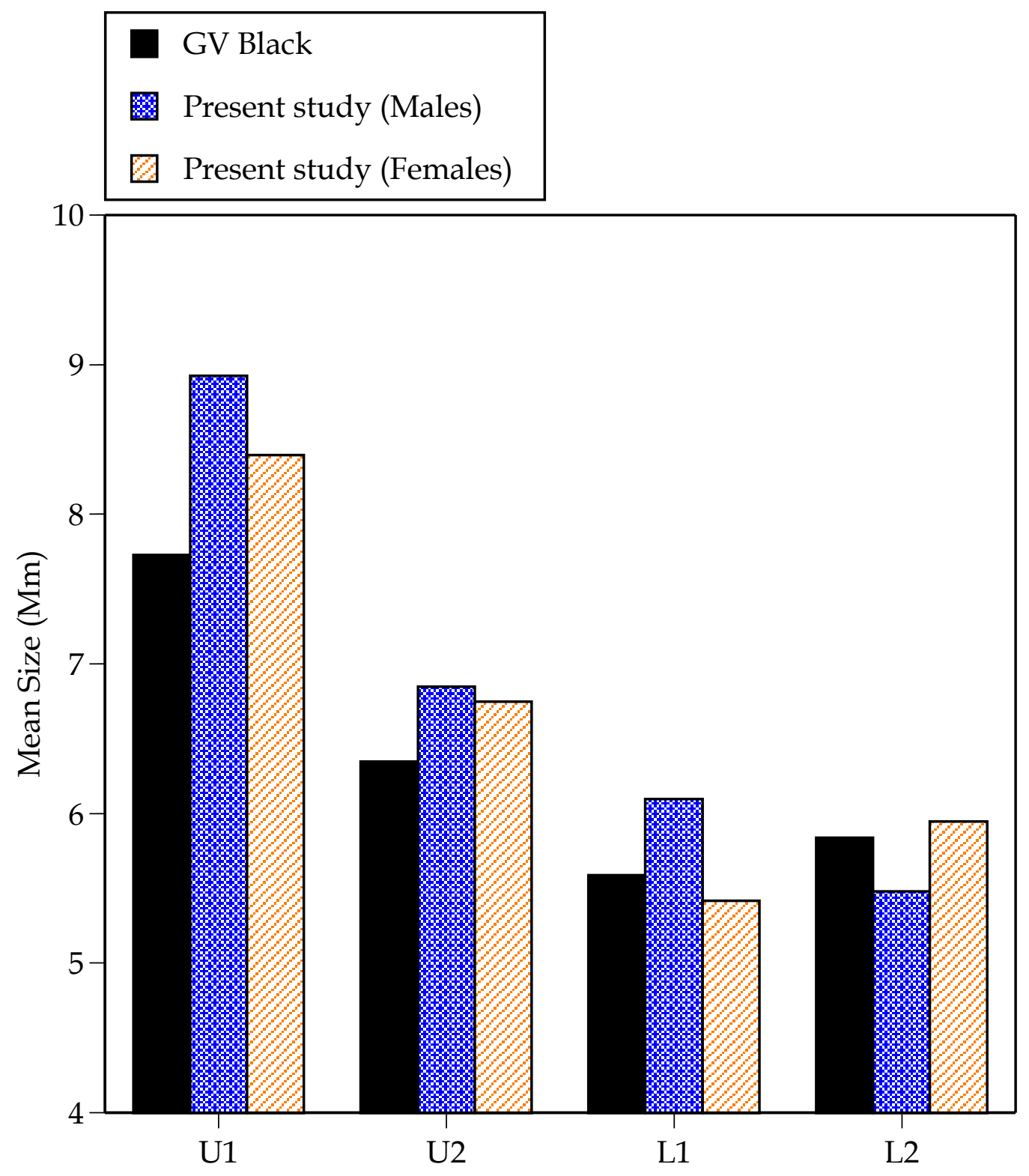

Fig. 13. Comparisons of mesiodistal crown widths between G. V. Black's data and the present study (where data are presented by sex). 
width, tooth length, pulp height, and alveolar crestal bone levels from periapical radiographs.

Because of the high prevalence of malocclusion in the modern U.S. population and the increased demand for an esthetically pleasing smile, the frequency of orthodontic treatment is on the rise. And, there is considerable evidence that orthodontic tooth movement can cause external apical root resorption (Harris 2000), our size standards may help us better understand the nature of this iatrogenic effect.

\section{Left-Right Symmetry}

We found that distal root lengths are shorter than their mesial counterparts determined on each tooth measured. For each of the four tooth types measured, the distal root lengths were shorter than the mesial root lengths (Table 5). This may be the result of the high frequency of root curvature to the distal (Wheeler 1974). Since our calculations were determined using linear measurements, the more curved the root from the CEJ to the apex, the shorter the root length would be on the side of the curvature (Figure 1). Another explanation for this asymmetry in root lengths for each tooth type may be the curvature of the CEJ around the tooth. We know the CEJ is more coronal on the mesial and distal surfaces and more apical on the labial and lingual surfaces of an incisor tooth. Also, the mesial CEJ is slightly higher (more coronal) than the 
distal CEJ (a difference of about $1 \mathrm{~mm}$ ), so the researcher would expect this to increase the tooth's root measurement on the mesial of an incisor (Wheeler 1974).

The same phenomenon was found with respect to bone heights. The mesial bone heights were larger than the distal bone heights for all tooth types measured (Table 6). Once again, incisor root curvature to the distal may effect these linear measurements. Bone heights are expected to follow the natural curvature of the CEJ and be located within 2 to 3 millimeters of it (Moloff and Stein 1982). More relevant to the present study, in the healthy individual, the underlying crestal bone is usually 1.0 to $1.5 \mathrm{~mm}$ apical to the radiographic cementoenamel junction (Rose 2004).

However, our results of paired t-tests that assessed whether crestal bone heights were equivalent on the medial and lateral aspects of a tooth (Table 21) reported the dimension larger on the tooth's mesial aspect in three of the four tooth types. These calculations were made by subtracting crestal bone heights into root lengths, and root length was determined relative to the CEJ. If it is known that the mesial CEJ is a millimeter higher (more incisal) than the distal CEJ (Wheeler 1974), this observed greater mesial dimension may be explained. Only the maxillary lateral incisor distal bone height (1.27) was larger than its mesial counterpart mean (1.20). So, the other three measurements disclose that the bone is lower (more apical) on the incisor's mesial than its distal aspect. This finding would not necessarily mean that the distal bone height is larger than the 
mesial bone height, since anatomically, the CEJ is about a millimeter more incisal on the mesial than the distal four all four incisor tooth types (Wheeler 1974).

Bergström and coworkers (1986) likewise measured roots and bone heights as the mean distances from the mesial and distal CEJs and adjacent crestal bone to the apex. Their study did not evaluate mesial and distal bone height symmetry for each tooth, but focused on alveolar bone height as a percentage of root length. For the youngest age group of their study (21-30 years old; $\mathrm{n}=47$ ), the mean alveolar bone height as a percentage of root length was $87.4 \%$. This was the overall calculation for every mesial and distal measurement for every tooth type in the mouth (i.e., incisors, canines, premolars and molars) and in both arches (i.e., maxillary and mandibular) combined. The present study can be related to Bergström's by comparing to the data in Table 21, expressed as a percentage of root length. The averaged bone height for all four tooth types was 1.0875 which can be converted to $91.95 \%$. Our mandibular incisor values were greatest at $109.59 \%$, meaning that the bone height was higher (more coronal) than the CEJ at times. This may be the result of errors in the radiographic technique.

$\underline{\text { Sexual Dimorphism }}$

This study discovered from the four incisor types crown widths that just the maxillary central and mandibular lateral incisor tooth types exhibited 
significant dimorphism, but only on the order of 2 to $3 \%$. Garn and coworkers (1967) documented the same level of dimorphism for another group of modern American Whites (Ohio), averaging about 4.2\% dimorphic for all tooth types (i.e. incisors, canines, premolars, and molars). Others report that mesiodistal crown dimensions showed little significant sexual dimorphism except for the maxillary central and mandibular lateral incisors, and this is true only when the canines are excluded, which are more dimorphic than the incisors (Townsend 1979; Harris and Bailit 1987).

Harris and Hicks (1998) studied periapical radiographs of the four maxillary incisor crowns and reported that sexual dimorphism was related to dentine differences between the sexes instead of enamel thicknesses. This dimorphism averaged about $6.5 \%$ in the American white sample, and it was slightly higher in the lateral incisor than the central incisor and slightly higher for the width of the dentine than the width of the whole tooth.

Woods et al. (1990) found that males have significantly longer roots than females; also, for the incisor, a person's sex is a more important determinant of root length than race (i.e., American black or white). Root length dimorphism was $4 \%$ for the maxillary central incisors in their study. We documented incisor root length to be sexually dimorphic, with males averaging 5 to $7 \%$ longer roots than the females. Also, for crown heights, the maxillary lateral incisor was the only tooth of the four studied to show significant dimorphism. Males had crown 
heights that, on average, were $3.6 \%$ larger in this instance.

\section{Effect of Angle's Classification}

The present study discovered incisor tooth size difference among Angle's classes. Specifically, maxillary lateral incisors are smaller in the Class I sample. Indeed, the maxillary lateral incisor yields substantive evidence of a size difference among Angle's Classes, with Class I cases (controlling for sex) having shorter root dimensions than Class II cases (Appendix: Figures A-1 and A-2).

Araujo and Souki (2003) studied Bolton anterior tooth size discrepancies among different malocclusion groups. An anterior Bolton discrepancy relates the mandibular anterior crown widths to the maxillary anterior crown widths as discussed previously. Clinically, this study is important to the orthodontist, because a Bolton anterior tooth size discrepancy can dictate the coupling of the dental arches, proper anterior guidance of the occlusion, and dental esthetics. If there is a difference relating to this discrepancy among the Angle's classes, the orthodontist could plan treatment to intercept these problems. From their study, Araujo and Souki concluded that individuals with Class I and Class III malocclusions had significantly greater frequencies of tooth size discrepancies than individuals with Class II malocclusions. Also, the mean anterior tooth size discrepancy for Angle Class III subjects was much greater than for Class I and 
Class II subjects. In relation to the present study, the fact that the maxillary lateral incisor was slightly narrower mesiodistally in the Class I sample would support the findings of Araujo and Souki, which may lead to a greater chance of a mandibular anterior Bolton excess for the Class I malocclusion than the Class II malocclusion. If the sum of the maxillary anterior teeth crown widths is less than normal, there will be difficulty in closing all of the space between the upper incisors and maintaining an ideal Class I canine/molar buccal segment relationships.

Peck et al. (1998) reported all four incisor mesiodistal crown diameters studied (i.e., maxillary left central, maxillary left lateral, mandibular left central and mandibular left lateral) were narrower on average in their Class II division 2 group than their control group. This control group was determined in a previous study which involved a survey $(n=537)$ to determine the prevalence of the Class II division 2 malocclusion in an orthodontic population (Peck and Peck 1979). Once again, the maxillary central and mandibular lateral incisors were significantly narrower mesiodistally in the Class II/2 group. In relation to orthodontic clinical relevance, given a pattern of reduced mesiodistal tooth size and well-developed jaw size in the Angle's Class II/2 patient, it is understood that an adequate dental arch length and reduced tooth width would not require permanent tooth extractions for correction (Arvystas 1990). The present study grouped Class II, division 1 and Class II/2 samples due to insufficient sample 
sizes individually. Today, in studies of dentofacial treatment and growth, investigators tend to combine the Class II/1 and II/2 together, but Peck and coworkers believe they should be kept separate according to Angle's design. Grouping into one Class II category could result in potential drawbacks in the research design and skew the results of the study.

Lavelle (1972) evaluated maxillary and mandibular crown sizes among racial groups and in different occlusal categories. He discovered that the overall tooth crown dimensions were greatest in Class I and least in Class III for the maxillary dentition; however Class II divisions 1 and 2 were intermediate. His results suggest that tooth size may play a role in the etiology of malocclusions. Tooth size is an important factor to be considered in orthodontic diagnosis and treatment.

\section{Crown Heights and Root Lengths}

Developmentally, the average crown height of the fully erupted maxillary central incisor in the adult patient is 9.5 to $11.0 \mathrm{~mm}$ (Gillen et al. 1994). Central incisors of shorter length do occur; however, the teeth tend to be proportionally narrower so that crown width generally is 75 to $80 \%$ of the crown height. The present study calculated crown and root dimensions in adolescents, so the incisor teeth are fully erupted into the occlusion yet there is little chance of tooth wear. 
The present study reported anatomic crown height from the periapical radiographs by subtracting overall tooth length by the root length. This was not a study using extracted teeth, and our perspective is that this calculated crown height would be more accurate than measuring the clinical crown heights on the casts. Magne et al. (2003) measured extracted maxillary anterior human teeth, and they reported that cast measurements for clinical crown height by Sterrett et al. (1999) were on average $1 \mathrm{~mm}$ shorter than their extracted anatomic crown heights. So, they contended that crown height measurements on dental casts were not as accurate as those made from visibly identifying the CEJ.

The present study found that the maxillary central incisor crown heights and root lengths were larger than those of the maxillary lateral incisor. In contrast, in the mandibular arch, the central incisor crown height was larger than the lateral, but the lateral incisor root length was larger than the central. This was true of our entire sample size combined. Also, regarding crown heights, we discovered both mandibular incisor types had virtually identical mean sizes in females than males (Table 17).

Kramer and Ireland (1959) measured extracted primary anterior teeth in harmony with G. V. Black's (1897) measurement design. It is interesting to comment on the closeness of their data to Black's. Maxillary central incisor root length was slightly longer than maxillary lateral incisor root length measuring (10.3 mm and $10.2 \mathrm{~mm}$, respectively). Upper incisor crown height was greater 
for the central by an average of $0.5 \mathrm{~mm}$ over the lateral incisor. As in the present study, the reverse was found to be true for the mandibular incisor dimensions, where the lateral root lengths averaged $1.0 \mathrm{~mm}$ greater than the central roots. Differing from the present study, mandibular lateral crown heights were taller on the average than the centrals by about $0.3 \mathrm{~mm}$.

Morrow and coworkers (2000) investigated the relationship between age, gender and clinical crown length using a longitudinal study design. Clinical crown heights were measured from casts using sliding calipers. They focused on the process of passive eruption, which resulted in progressively increasing clinical crown length that continued to increase throughout the teenage years in their sample. The present study only measured crown height from the periapical radiographs and not the casts. The primary reasons are the uncontrolled variables (e.g., gingival inflammation, gingival overgrowth, and tissue thickness) making it difficult to obtain the clinical crown height. Anatomic crown height may be found more accurately from radiographs. In relation to continued passive eruption or apical migration of the gingival margin, using anatomic crown height instead of clinical crown height avoids that uncontrolled source of variation.

Clinically, it is important to consider the patient's age when finishing a case that needs anterior restorations after orthodontic treatment. Since Morrow and coworkers (2000) reported a $0.5 \mathrm{~mm}$ change in the clinical crown lengths of 
the maxillary anterior teeth during the teenage years, the esthetics of restorations may be challenged. It may be advisable to delay final restorative treatment until after 19 years of age.

\section{Mesiodistal Crown Width Ratios}

The present study measured periapical radiographs and casts of nonextracted maxillary and mandibular incisors. Width measurements of extracted teeth are precise because of the proximal clearance (absence of neighboring teeth). In contrast, the precision of clinical measurements (including those made on casts) can be jeopardized because there are overlapping tooth margins that affect measurement accuracy. This could explain why average widths in the study by Sterrett et al. (1999) were $1 \mathrm{~mm}$ larger compared to clinical crown measurements made from dental casts.

Because of the absence of sexual dimorphism in crown width ratios, we combined the sexes and Angle's malocclusions and calculated the maxillary lateral to central ratio to be 0.771 and the mandibular ratio to be 1.105 . Since we measured only incisor teeth, we could not calculate our sample's Bolton anterior ratios. Smith et al. (2000) reported that there was no statistical difference in Bolton ratios between males and females, and this would be in harmony with our findings since sexual dimorphism related to crown width proportionalities (i.e. U2/U1 and L2/L1) was absent in our sample. 
Ballard (1956) measured 500 sets of casts and compared the crown widths of the teeth on each side of the dental arch. He advocated interproximal reduction of the teeth, especially in the anterior segment, when there was a lack of balance between opposing arches. Bolton $(1958,1962)$ compared the crown widths of the maxillary and mandibular dentitions of 55 cases and formulated tables to predict the congruity between the two arches.

Deviant mesiodistal crown width ratios can pose a problem when finishing an orthodontic case. Most often the maxillary lateral incisor is too narrow, which makes it difficult for the orthodontist to close all of the space in the upper arch and have proper anterior coupling. This problem may be resolved by interproximal reduction of the lower incisors or restoring the small maxillary laterals to normal width by the general dentist (i.e., direct composite bonding, full coverage porcelain crowns, or veneers). The present study was able to look at this ratio from intra-arch relationships (U1/U2 and L1/L2) rather than a conventional Bolton analysis that is calculated from inter-arch relationships (sum of the six mandibular anterior crown widths/sum of the six maxillary anterior crown widths).

Another factor of anterior crown widths researched more commonly in restorative dentistry is the concept of the golden proportion. According to this concept, the perceived width of the maxillary lateral incisor should be approximately $62 \%$ of the perceived width of the central incisor, whereas that of 
the canine should be similarly related to the lateral incisor. So if the width of the maxillary lateral incisor is $1 \mathrm{~mm}$, the central incisor should be 1.618 times wider and the canine 0.618 times narrower (Ward 2001; Gurel 2003). In the present study, the average ratio of the maxillary central incisor to the lateral incisor was 1.310. This observed ratio (1.310) seems much smaller than the 1.618 predicted from the divine proportion. This difference also is readily confirmed statistically: The 95\% confidence limits of the observed ratio (1.310) are 1.293 and 1.327, and these limits clearly do not include the anticipated divine proportion of 1.618. Similarly, testing whether the observed ratio is statistically the same as the divine ratio of 1.618, yields a one-sample t-test of 35.8, which suggests that the two ratios (observed and expected) are hugely different. Other dental researchers have arrived at this same conclusion - that $\mathrm{U} 1$ is broader than $\mathrm{U} 2$, but the ratio between them is much smaller than the divine proportion.

\section{Crown-Root Ratios}

Our study found that crown heights of the maxillary central incisors were statistically the same between the sexes, so the observed differences in the crown-root ratios are attributed predominantly to shorter roots in females than males. Root length was found to be $49 \%$ of the crown height for U1 in females, but slightly less, $47 \%$, in males. Both mandibular incisor teeth had significantly higher crown-root ratios in females than males, mainly due to the shorter root 
lengths and also taller crown heights in females. In general, for all incisor tooth types combined, crown heights are slightly taller in males while root lengths are disproportionately long, so females have greater crown-root ratios.

Eliasson et al. (1986) looked at the relationship between root length and overall tooth length. The relationship was usually greater on the mesial aspect than on the distal. The differences between males and females were small $(<$ $2.9 \%)$. The mean root length related to tooth length was $64.5 \%$ and $66.9 \%$ in the maxilla and mandible, respectively.

\section{$\underline{\text { Alveolar Bone Heights }}$}

In the healthy individual, the underlying crestal bone is usually 1.0 to 1.5 mm apical to the radiographic cementoenamel junction (Rose 2004). Radiographic assessment of bone heights can be determined by the distance from the alveolar crestal bone to the CEJ of a tooth. Bone loss can be expressed as this distance in millimeters or as a percentage of the root length (Carranza 2002). The three main sources of error in assessment of bone heights with radiographs are (1) variation in projection geometry of the x-ray beam to the film, (2) variations in film contrast and density, and (3) obstruction of the view by other anatomic structures (Armitage 1996). In the present study the alveolar crestal bone was about a millimeter apical to the CEJ. All of the means in the maxilla exceeded a millimeter (averaging $1.26 \mathrm{~mm}$ ), while the average of the 
means in the mandible was less than a millimeter $(0.92 \mathrm{~mm})$. The present study located the mesial and distal radiographic CEJs by plotting the junction of the radiopaque enamel to the more radiolucent cementum (Figure 1).

Results of paired t-tests (Table 21) assessing whether crestal bone heights were equivalent on the medial and lateral aspects of the incisor teeth, confirmed that three of the four incisors had larger dimensions on the mesial than the distal. However, this does not mean the crestal bone levels are higher (more coronal) on the distal because the CEJ is located a millimeter higher on the mesial of the incisor, too. In the healthy patient, the mesial and distal bone heights are fairly horizontal from tooth to tooth in the dental arch, so our findings of larger mesial dimensions only supports the fact that the CEJ is more coronally located on the mesial of the incisor tooth than on the distal. For example, the maxillary central incisor had a mean difference of $-0.25 \mathrm{~mm}$, so the distance from the CEJ to the crestal bone was only a quarter of a millimeter greater on the mesial than on the distal. This was the largest difference of the four tooth types measured. 


\section{CHAPTER VI}

\section{SUMMARY AND CONCLUSIONS}

The present study established contemporary metric crown and root standards on adolescents prior to orthodontic treatment using measurements from periapical radiographs and dental casts. The sample size consisted of 148 American White adolescents, 51 males and 97 females, examined between the ages of 9 to 19 years of age. The intent of our study was to better understand incisor crown and root dimensions of adolescents prior to orthodontics, so that subsequent research may compare posttreatment data and recognize the severity of external apical root resorption.

1. Of the four incisor tooth types, only the maxillary central incisor mesiodistal crown dimension exhibited significant sexual dimorphism, at about $4 \%$.

Percentagewise, mean crown widths for males was only 1 to $2 \%$ larger than for females.

2. Crown heights exhibited comparably little sexual dimorphism. Only the mean size difference for the maxillary lateral incisor was significant statistically (a 4\% difference), and crown heights of the mandibular incisors were virtually identical in the two sexes.

3. Given that tooth length was composed of crown height and root length and, since sex differences in crown height were minor, most of the dimorphism 
was due to sex differences in root length. This dimorphism in incisor root lengths was in the range of 5 to $8 \%$, which is noticeably higher than for crown widths or heights.

4. Crown-root ratios for all tooth types were on the order of $50 \%$, showing that root length was about twice the crown height. Mean crown-root ratios were slightly larger in the mandible because the mandibular root lengths were proportionately shorter.

5. Distal root lengths and bone heights were systematically shorter than the corresponding mesial root lengths and bone heights for all four tooth types. This was probably due to the high frequency of root curvature to the distal of the incisor tooth types. Also, because the CEJ undulates around the circumference of the tooth (and because it is located about a millimeter more coronal on the mesial than the distal), distal measurements are shorter than mesial measurements.

6. Alveolar crestal bone heights were about a millimeter apical to the tooth's cementoenamel junction. All of the means for bone height in the maxilla exceeded a millimeter, while most of the means in the mandible were less than a millimeter.

7. Only the maxillary lateral incisor yielded substantive evidence of overall tooth size difference between Angle's Classes, with Class I cases (controlling for sex) having smaller root dimensions than the Class II cases. 


\section{LIST OF REFERENCES}


Alling CC, Helfrick JF, Alling RD. Impacted teeth. Philadelphia: W.B. Saunders Company, 1993.

Anderson DL, Thompson GW, Popovich F. Tooth, chin, bone and body correlations. Am J Phys Anthropol 1977;46:7-12.

Araujo E, Souki M. Bolton anterior tooth size discrepancies among different malocclusion groups. Angle Orthod 2003;73:307-13.

Arvystas MG. Nonextraction treatment of severe Class II, division 2 malocclusions. Am J Orthod Dentofacial Orthop 1990;97:510-21.

Ash MM Jr. Wheeler's dental anatomy, physiology, and occlusion, 7th ed. Philadephia: W.B. Saunders, 1993.

Armitage GC. Periodontal diseases: diagnosis. Ann Periodontol 1996;1:37-215. Baccetti T. A controlled study of associated dental anomalies. Angle Orthod $1998 ; 68: 267-74$.

Ballard ML. Asymmetry in tooth size: a factor in the etiology, diagnosis and treatment of malocclusion. Angle Orthod 1944;68:67-70.

Basdra EK, Kiokpasoglou MN, Komposch G. Congenital tooth anomalies and malocclusions: a genetic link? Eur J Orthod 2001;23:145-51.

Basdra EK, Kiokpasoglou MN, Stellzig A. The Class II division 2 craniofacial type is associated with numerous congenital tooth anomalies. Eur J Orthod 2000;22:529-35.

Beck BW, Harris EF. Apical root resorption in orthodontically treated subjects: 
analysis of edgewise and light wire mechanics. Am J Orthod Dentofacial Orthop 1994;105:350-361.

Beck BW, Harris EF. External apical root resorption in orthodontic patients. Frater of Psi Omega 1992;92:11-3.

Bergström J, Eliasson S. Alveolar bone height in professional musicians. Acta Odontol Scand 1986;44:141-7.

Bishara SE, Vonwald L, Jakobsen JR. Changes in root length from early to midadulthood: Resorption or apposition? Am J Dentofacial Orthop 1999;115:563-8.

Björn H, Holmberg K. Radiographic determination of periodontal bone destruction in epidemiological research. Odontologisk Revy 1966;17:23250.

Black GV. Descriptive anatomy of the human teeth, 4th ed. Philadelphia: S.S. White, 1897.

Blackith RE, Reyment RA. Multivariate morphometrics. New York: Academic Press, 1971.

Bolton WA. The clinical application of tooth-size analysis. Am J Orthod 1962;48:504-29.

Bolton WA. Disharmony in tooth size and its relation to the analysis and treatment of malocclusion. Angle Orthod 1958;28:113-30.

Bowers CM, Bell GL. Manual of forensic odontology, 3rd ed. Washington, DC: 
American Society of Forensic Odontology, 1995.

Bredy E, Erbing C, Hubenthal B. Haufigkeit der Zahnunterzahl bei Anlage und nichtanlage von Weisheitzahnen. Deutsche Zahn-Mund-Kieferheilkunde 1991;19:357-63.

Bryant RM, Sadowsky PL, Hazelrig JB. Variability in three morphologic features of the permanent maxillary central incisor. Am J Orthod 1984;86:25-32.

Carlson H. Studies on the rate and amount of eruption of certain human teeth. Am J Orthod Oral Surg 1944;30:575-88.

Carranza FA, Newman MG, Takei HH. Carranza's clinical periodontology, 9th ed. Philadelphia: W.B. Saunders Company, 2002.

Chen SK, Pan JH, Chen CM, Jeng JY. Accuracy of supported root ratio estimation from projected length and area using digital radiographs. J Periodontol 2004;75:866-71.

Cooley WW, Lohnes PR. Multivariate data analysis. New York: John Wiley \& Sons, Inc., 1971.

Crosby DR, Alexander CG. The occurrence of tooth size discrepancies among different malocclusion groups. Am J Orthod 1972;61:29-37.

Cua-Benward GB, Dibaj S, Ghassemi B. The prevalence of congenitally missing teeth in Class I, II, III malocclusions. J Clin Pediatr Dent 1992;17:15-7.

Dachi SF, Howell FV. A study of impacted teeth. Oral Surg Oral Med Oral Pathol 1961;14:1165-9. 
Dahlberg AA. The changing dentition of man. J Am Dent Assoc 1945;32:676-90.

Darling AI, Levers BGH. The pattern of eruption of some human teeth. Arch Oral Biol 1975;20:89-96.

Debrot A. Time interval between eruption of homologous teeth. J Dent Res 1969;48:135-71.

Delivanis HP, Kuftinec MM. Variation in morphology of the maxillary central incisors found in Class II, division 2 malocclusions. Am J Orthod 1980;78:438-43.

Dermaut LR, De Munck A. Apical root resorption of upper incisors caused by intrusive tooth movement: a radiographic study. Am J Orthod 1986;90:321-6.

Ditch LE, Rose JC. A multivariate dental sexing technique. Am J Phys Anthropol 1972;37:61-4.

Doris JM, Bernard BW, Kuftinec MM, Stom D. A biometric study of tooth size and dental crowding. Am J Orthod 1981;79:326-36.

Eliasson S, Lavstedt S, Ljungheimer C. Radiographic study of alveolar bone height related to tooth and root length. Comm Dent Oral Epidemiol 1986;14:169-71.

Ericsson I, Lindha J. Effect of longstanding jiggling on experimental marginal periodontitis in the beagle dogs. J Clin Periodontol 1982;9:497-503. 
Farmer V. Variability in the deciduous dentition: an odontometric and morphologic study of a group of South Australian children. M.S. thesis, Dentistry, University of Adelaide, South Australia, 1990.

Gantt DG, Harris EF, Rafter JA, Rahn JK. Distribution of enamel thickness on human deciduous molars. In: Brooks A, editor. Dental morphology 2001. Sheffield, England: Sheffield Academic Press, 2001, p. 167-90.

Garn SM, Cole PE, Van Alstine WL. Sex discriminatory effectiveness using combinations of root lengths and crown diameters. Am J Phys Anthropol 1978a;50:115-8.

Garn SM, Lewis AB. The relationship between third molar agenesis and reduction in tooth number. Angle Orthod 1962;32:14-8.

Garn SM, Lewis AB, Kerewsky RS. Third molar agenesis and size reduction of the remaining teeth. Nature 1963;200:488-9.

Garn SM, Lewis AB, Kerewsky RS. Sex difference in tooth size. J Dent Res 1964;43:306-7.

Garn SM, Lewis AB, Kerewsky RS. Third molar agenesis and variation in size of the remaining teeth. Nature 1964;201:839.

Garn SM, Lewis AB, Kerewsky RS. The meaning of bilateral asymmetry in the permanent dentition. Angle Orthod 1966;36:55-62.

Garn SM, Lewis AB, Swindler DR, Kerewsky RS. Genetic control of sexual dimorphism in tooth size. J Dent Res 1967;46:963-72. 
Garn SM, Smith BH, Cole PE. Correlations between root length and face size. J Dent Res 1980;59:141.

Garn SM, Van Alstine WL Jr, Cole PE. Intraindividual root-length correlations. J Dent Res 1978b;57:27.

Garn SM, Van Alstine WL Jr, Cole PE. Relationship between root lengths and crown diameters of the corresponding teeth. J Dent Res 1978c; 57:636.

Garn SM, Van Alstine WL, Cole PE. Root-length and crown-size correlations in the mandible. J Dent Res 1978;57:114.

Gillen RF, Schwartz RS, Hilton TJ, et al. An analysis of selected normative 410tooth proportions. Int J Pros 1994;7:410-7.

Gilpatric WH. Arch predetermination, is it practical? J Am Dent Assoc 1923; 10:553-72.

Goaz PW, White SR. Oral Radiology, 2nd ed. St. Louis: C.V. Mosby, 1987.

Goodson JM, Haffajee AD, Socransky SS. The relationship between attachment level loss and alveolar bone loss. J Clinic Perio 1984;11:348-59.

Gurel G, editor. The science and art of porcelain laminate veneers. London: Quintessence, 2003, p 83-6.

Gustafson G. Age determination on teeth. J Am Dent Assoc 1950;41:45-54.

Harman HH. Modern factor analysis, 3rd ed. Chicago: University of Chicago Press, 1976.

Harris EF. External apical root resorption resulting from orthodontic tooth 
movement. Semin Orthod 2000;6:183-94.

Harris EF, Baker WC. Loss of root length and crestal bone height before and during treatment in adolescent and adult orthodontic patients. Am J Orthod Dentofacial Orthop 1990;98:463-9.

Harris EF, Burris BG. Contemporary permanent tooth dimensions with comparisons to GV Black's data. J Tennessee Dent Assoc 2003;83:25-9.

Harris EF, Butler ML. Patterns of incisor root resorption before and after orthodontic correction in cases with anterior openbites. Am J Orthod Dentofacial Orthop 1992;101:112-119.

Harris EF, Couch WM. The relative sexual dimorphism of human incisor crown and root dimensions. Dental Anthropology 2006;19:87-95.

Harris EF, Hassankiadeh S, Harris JT. Maxillary incisor crown-root relationships in different Angle malocclusions. Am J Orthod Dentofacial Orthop 1993;103:48-53.

Harris EF, Hicks JD. Enamel thickness in maxillary human incisors: a radiographic assessment. Arch Oral Biol 1998;43:825-31.

Harris EF, Hicks JD, Barcroft BD. Tissue contributions to sex and race: differences in tooth crown size of deciduous molars. Am J Phys Anthropol 2001;115:223-37.

Harris EF, Kineret SE, Tolley EA. A heritable component for external apical root resorption in patients treated orthodontically. Am J Orthod Dentofacial 
Orthop 1997;111:301-9.

Harris EF, McKee JH. Tooth mineralization standards for Blacks and Whites from middle southern United States. J Forensic Sci 1990;35:859-72.

Harris EF, McKee JH. Tooth mineralization standards for Blacks and Whites from the midsouth United States. In: Bowers EM, Bell GL, eds. Manual of forensic odontology, 3rd ed. Publication of the American Society of Forensic Odontology 1995; Montpelier, VT: Printing Specialists, 1995, p. $92-8$.

Harris EF, Rathbun TA. Ethnic differences in the apportionment of tooth sizes. In: Kelley MA, Larsen CS, editors. Advances in dental anthropology. New York: Alan R. Liss, Inc., 1991, p 121-42.

Harris EF, Woods MA, Robinson QC. Dental health patterns in an urban midsouth population: race, sex and age changes. Quintessence Int 1992;24:45-52.

Haruki T, Kanomi R, Shimono T. The differences in the chronology and calcification of second molars between angle Class III and Class II occlusions in Japanese children. ASDC J Dent Child.1997;64:400-4.

Hasanreisoglu U, Berksun S, Aras K, Arslan I. An analysis of maxillary anterior teeth: facial and dental proportions. J Prosthet Dent 2005;94:530-8.

Holtta P, Nystrom M, Evalahti M, Alaluusua S. Root-crown ratios of permanent teeth in a healthy Finnish population assessed from panoramic 
radiographs. Eur J Orthod 2004;26:491-7.

Howe RP, McNamara JA Jr, O'Connor KA. An examination of dental crowding and its relationship to tooth size and arch dimension. Am J Orthod 1983;83:363-73.

Hurme VO. Ranges of normalcy in the eruption of the first six permanent teeth. J Dent Child 1949;16:11-5.

Jeffcoat MK, Williams RC. Relationship between linear and area measurements of radiographic bone levels utilizing simple computerized techniques. J Periodontal Res 1984;19:191-8.

Kaiser HF. The varimax criterion for analytic rotation in factor analysis. Psychometrika 1958;23:187-200.

Kaley J, Phillips C. Factors related to root resorption in edgewise practice. Angle Orthod 1991;61:125-32.

Kelly JE, Harvey CR. An assessment of the occlusion of youth 12-17 years. United States Public Health Service, 1977 (Vital and health statistics series 11; no. 162).

Kieser JA. Human adult odontometrics: the study of variation in adult tooth size. New York: Cambridge University Press, 1990.

Kolakowski D, Bailit HL. Segregation analysis of tooth size in a Melanesian population. Ann Hum Biol 1981;8:255-62.

Kramer WS, Ireland RL. Measurements of the primary teeth. ASDC J Dent 
Child 1959;26:252-61.

Kullman L, Martinsson T. Computerized registration of epidemiological data from intraoral radiographs. Swed Dent J 1985;9:89-96.

Laino A, Quaremba G, Paduano S, Stanzione S. Prevalence of tooth-size discrepancy among different malocclusion groups. Prog Orthod 2003;4:37-44.

Lamendin H, Baccino E, Humbert JF, Tavernier JC, Nossintchouk RM, Zerilli A. A simple technique for age estimation in adult corpses: the two criteria dental method. J Forensic Sci 1992;37:1373-9.

Langland OE, Sippy FH, Langlais RP. Textbook of dental radiology: Charles C. Thomas, Springfield, Illinois, 1984.

Lapatki BG, Mager AS, Schulte-Moenting J, Jonas IE. The importance of the level of the lip line and resting lip pressure in Class II, division 2 malocclusion. J Dent Res 2002;81:323-8.

Lavelle CLB. Maxillary and mandibular tooth size in different racial groups and in different occlusal categories. Am J Orthod 1972;61:29-37.

Lavstedt S, Bolin A, Henrikson CO, Carstensen J. Proximal alveolar bon loss in a longitudinal radiographic investigation (I). Methods of measurement and partial recording. Acta Odontol Scand 1986;44:149-57.

Lavstedt S, Eklund G, Henrikson CO. Partial recording in conjunction with roentgenologic assessment of proximal marginal bone loss. Acta Odontol 
Scand 1975;33:90-113.

Levander E, Malmgren O. Evaluation of the risk of root resorption during orthodontic treatment: a study of upper incisors. Eur J Orthod 1988;10:30-8.

Liversidge H. Variation in modern human dental development. In: Thompson JL, Krovitz GE, Nelson AJ, eds. Patterns of growth and development in the genus $\underline{\text { Homo }}$. Cambridge: Cambridge University Press, 2003, p 73113.

Lundstöm A. Intermaxillary tooth width ratio and tooth alignment and occlusion. Acta Odontolol Scand 1954;12:265-92.

Lundström A. Some asymmetries of the dental arches, jaws and skull, and their etiological significance. Am J Orthod 1961;47:81-106.

Magne P, Gallucci GO, Belser UC. Anatomic crown width/length ratios of unworn and worn maxillary teeth in white subjects. J Prosthet Dent 2003;89:453-61.

Markkanen H, Rajala M, Knuuttila M, Lammi S. Alveolar bone loss in relation to periodontal treatment need, socioeconomic status and dental health. J Periodontol 1981;52:99-103.

McIntyre GT, Millett DT. Crown-root shape of the permanent maxillary central incisor. Angle Orthod 2003;73:710-5.

Mincer HH, Harris EF, Berryman HE. The A.B.F.O. study of third molar 
development and its use as an estimator of chronological age. J Forensic Sci 1993;38:379-90.

Mjör IA. Age changes in the teeth. In: Holm-Pedersen P, Löe H, eds. Geriatric dentistry: a textbook of oral gerontology. St. Louis: C.V. Mosby, 1986, p 94-101.

Moloff RL, Stein SD. Realities of dental therapy: a detailed review of periodontal prosthetic treatment. Chicago: Quintessence Publishing Company, Inc., 1982.

Moorrees CFA, Reed RB. Correlations among crown diameters of human teeth. Arch Oral Biol 1964;9:685-97.

Morrow LA, Robbins JW, Jones DL, Wilson NH. Clinical crown length changes from age 12-19 years: a longitudinal study. J Dent 2000;28:469-73.

Moyers RE. Handbook of orthodontics. Chicago: The Year Book Publishers, Inc., 1958.

Neff CW. Tailored occlusion with the anterior coefficient. Am J Orthod 1949;35:309-13.

Parker RJ, Harris EF. Directions of orthodontic tooth movements associated with external apical root resorption of the maxillary central incisor. Am J Orthod Dentofacial Orthop 1998;114:677-83.

Peck S, Peck H. Frequency of tooth extraction in orthodontic treatment. Am J Orthod 1979;76:491-6. 
Peck S, Peck L, Kataja M. Class II division 2 malocclusion: a heritable pattern of small teeth in well-developed jaws. Angle Orthod 1998;68:9-20.

Polson AM. Interrelationship of inflammation and tooth mobility (trauma) in pathogenesis of periodontal disease. J Clin Periodontol 1980;7:351-60.

Potter RH. Univariate versus multivariate differences in tooth size according to sex. J Dent Res 1972;51:716-22.

Proffit WR. Contemporary orthodontics, 3rd ed. St. Louis: C.V. Mosby, 2000.

Richardson ER, Malhotra SK. Mesiodistal crown dimension of the permanent dentition of American Negroes. Am J Orthod 1975;68:157-64.

Rose LF, Mealey BL, Genco RJ, Cohen DW. Periodontics: medicine, surgery, and implants. St. Louis: C.V. Mosby, 2004.

Saatci P, Yukay F. The effect of premolar extractions on tooth-size discrepancy. Am J Orthod Dentofacial Orthop 1997;111:428-34.

Salonen LW, Frithiof L, Wouter FR, Hellden LB. Marginal alveolar bone height in an adult Swedish population. A radiographic cross-sectional epidemiologic study. J Clin Periodontol 1991;18:223-32.

Schei O, Waerhaug J, Lovdahl A, Arno A. Alveolar bone loss as related to oral as related to oral hygiene and age. J Periodontol 1959;30:7-16.

Schultze C. Lehrbuch der Kieferorthopadie, vol. 3. Berlin: Quintessenz, 1982. Smith SS, Buchang PH, Watanabe E. Interarch tooth size relationships of 3 populations: “Does Bolton's analysis apply?" Am J Orthod Dentofacial 
Orthop 2000;117:169-74.

Sokal RR, Rohlf FJ. Biometry: the principles and practice of statistics in biological research, 3rd ed. San Francisco: W.H. Freeman and Company, 1995.

Stanton FL. Engineering in orthodontic diagnosis. J Am Dent Assoc $1928 ; 15: 825-32$.

Steggerda M, Hill TJ. Eruption time of teeth among Whites, Negroes and Indians. Am J Orthod 1942;28:361-70.

Sterrett JD, Oliver T, Robinson F, Fortson W, Knaak B, Russell CM. Width/length ratios of normal clinical crowns of the maxillary anterior dentition in man. J Clin Periodontol 1999;26:153-7.

Stramotas S, Geenty JP, Darendeliler MA, Byloff F, Berger J, Petocz P. The reliability of crown-root ratio, linear and angular measurements on panoramic radiographs. Clin Orthod Res 2000;3:182-91.

Suda N, Hiyama S, Kuroda T. Relationship between formation/eruption of maxillary teeth and skeletal pattern of maxilla. Am J Orthod Dentofacial Orthop 2002;121:46-52.

Swindler DR. Primate dentition: an introduction to the teeth of non-primates. Cambridge: Cambridge University Press, 2002.

Tanguay R, Demirjian A, Thibault HW. Sexual dimorphism in the emergence of the deciduous teeth. J Dent Res 1984;63:65-8. 
Thilander B, Jakobsson SO. Local factors in impaction of maxillary canines. Acta Odontol Scand 1968;26:145-68.

Townsend GC, Brown T. Inheritance of tooth size in Australian Aborigines. Am J Phys Anthropol 1978;48:291-3.

Tukey JW. Exploratory data analysis. Reading, Mass: Addision-Wesley, 1977. van der Linden FPGM, Duterloo HS. Development of the human dentition: an atlas. Hagerstown, MD: Harper and Row, Publishers, 1976.

Vaughan ME, Harris EF. Deciduous tooth size standards for American blacks. J Tenn Dent Assoc 1992;72:30-3.

Vlaskalic V, Boyd RL, Baumrind S. Etiology and sequelae of root resorption. Semin Orthod 1998;4:124-31.

Ward DH. Proportional smile design using the recurring esthetic dental (red) proportion. Dent Clin North Am 2001;45:143-54.

Wheeler RC. Dental anatomy, physiology and occlusion, 5th ed. Philadelphia: W.B. Saunders, 1974.

Winer BJ, Brown DR, Michels KM. Statistical principles in experimental design, 3rd ed. New York: McGraw-Hill Book Company, 1991.

Woods MA, Robinson QC, Harris EF. Age-progressive changes in pulp widths and root lengths during adulthood: a study of American blacks and whites. Gerodontology 1990;9:41-50.

Wouters FR, Salonen LW, Frithiof L, Hellden LD. Significance of some 
variables on interproximal alveolar bone height based on cross-sectional data. J Clin Periodontol 1993;20:199-206.

Zeisz RC, Nuckolls J. Dental anatomy. St. Louis: C.V. Mosby, 1949. 


\section{APPENDIX.}

HISTOGRAS OF MEAN TRAIT SIZES BY ANGLE'S CLASS AND SEX 


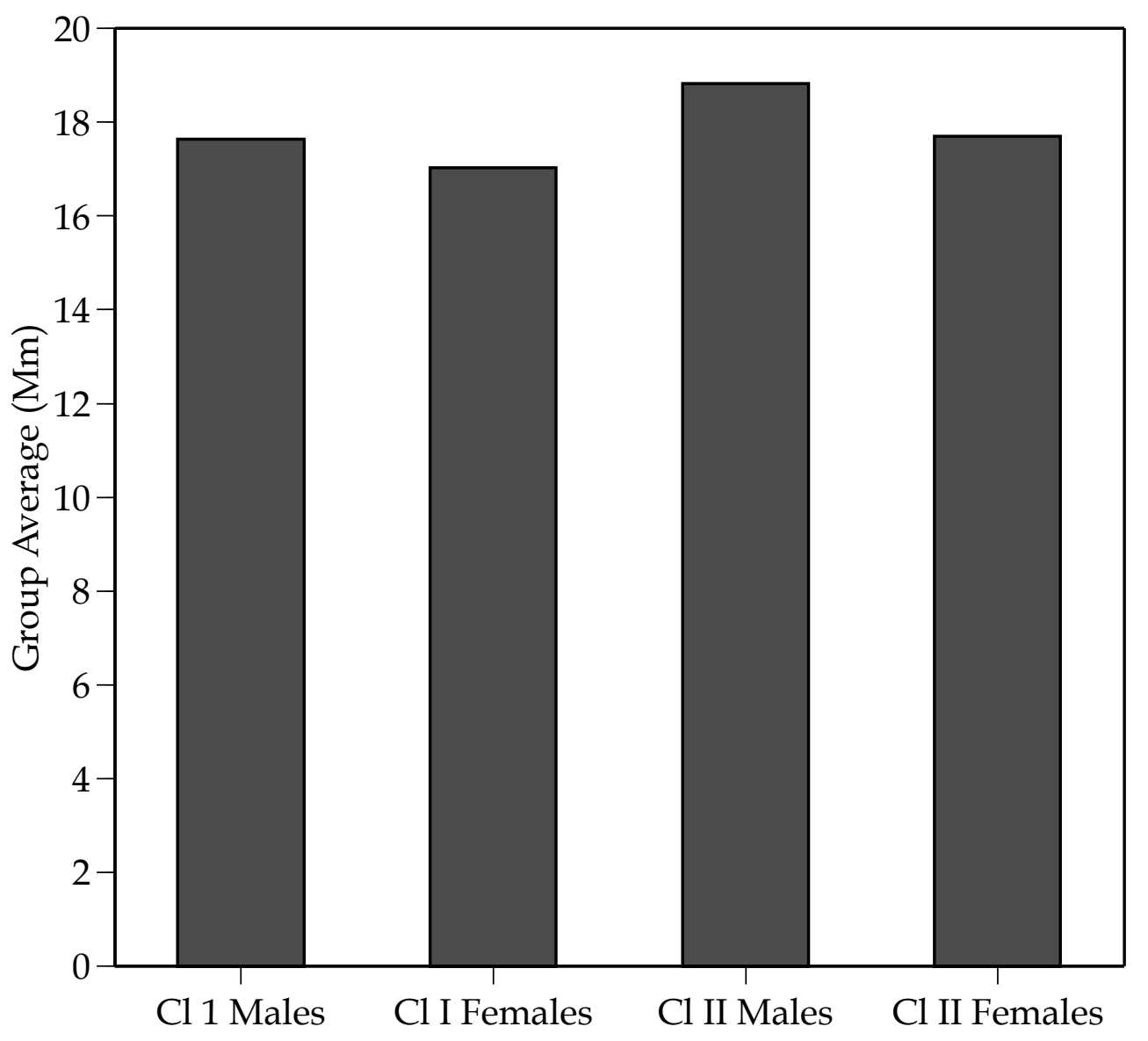

Fig. A-1. Group mean sizes, by Angle's classification and sex, for U2 mesial root length. 


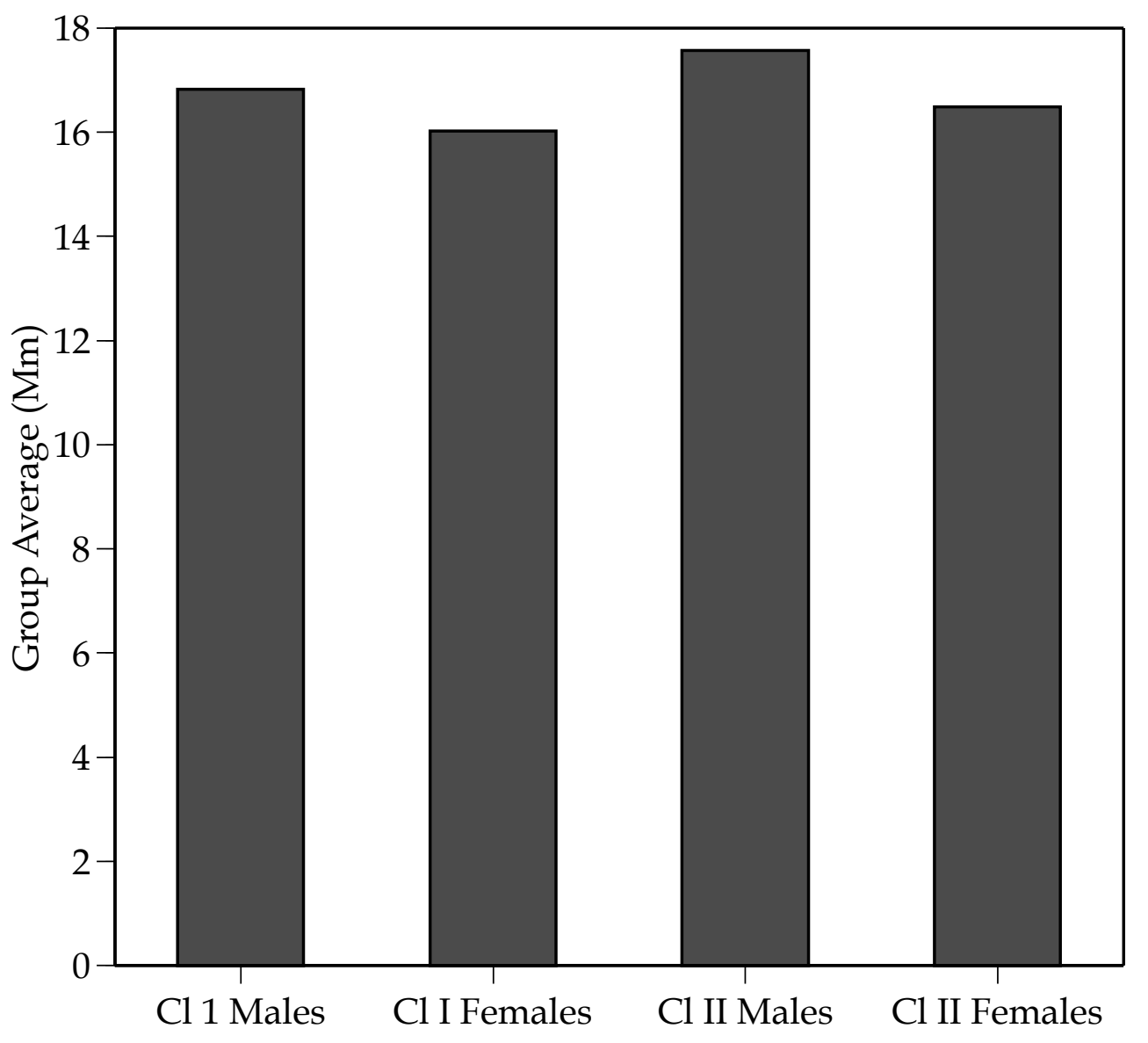

Fig. A-2. Group mean sizes, by Angle's classification and sex, for U2 distal root length. 


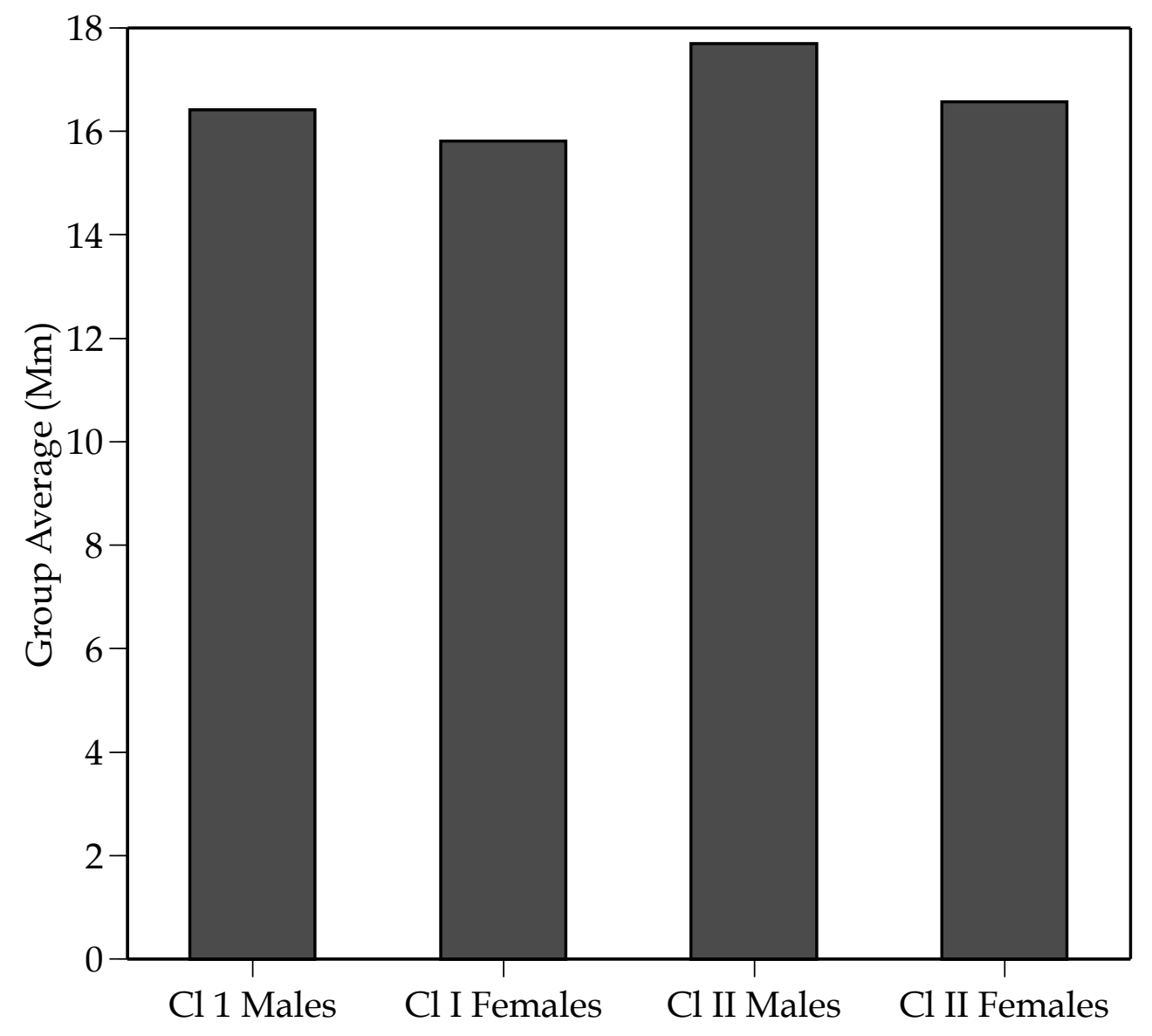

Fig. A-3. Group mean sizes, by Angle's classification and sex, for $\mathrm{U} 2$ mesial bone height. 


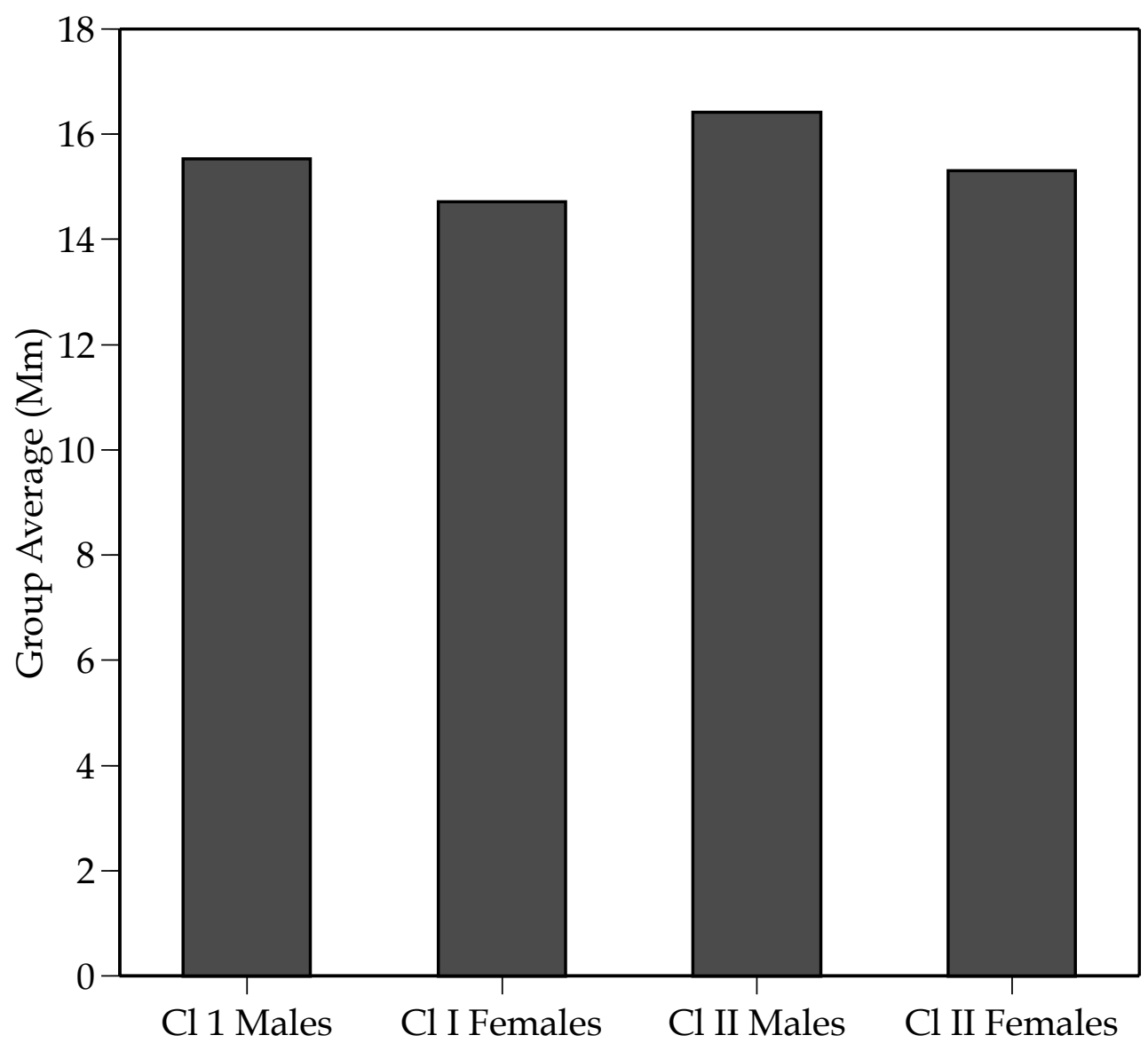

Fig. A-4. Group mean sizes, by Angle's classification and sex, for $\mathrm{U} 2$ distal bone height. 


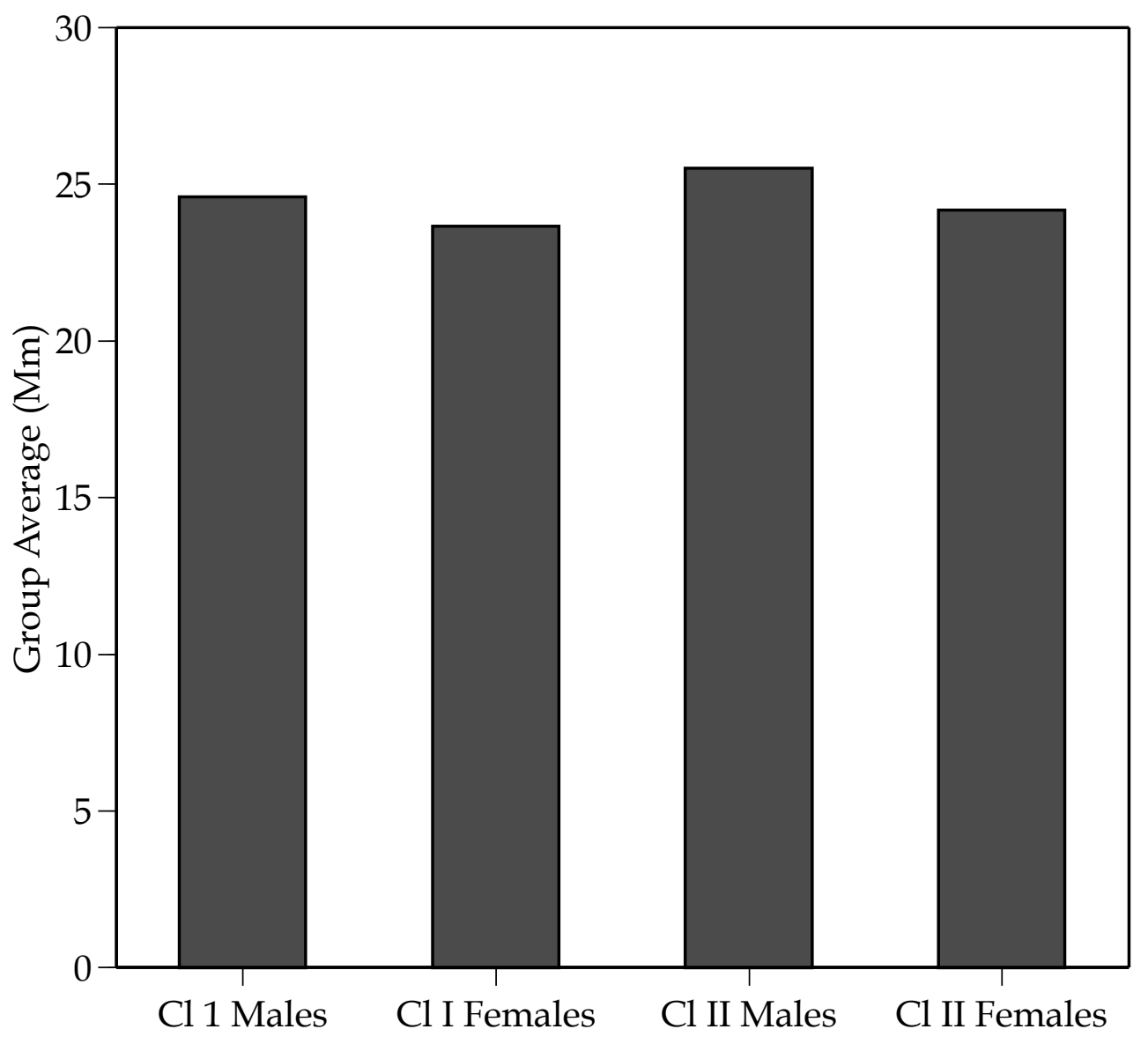

Fig. A-5. Group mean sizes, by Angle's classification and sex, for $\mathrm{U} 2$ tooth length. 


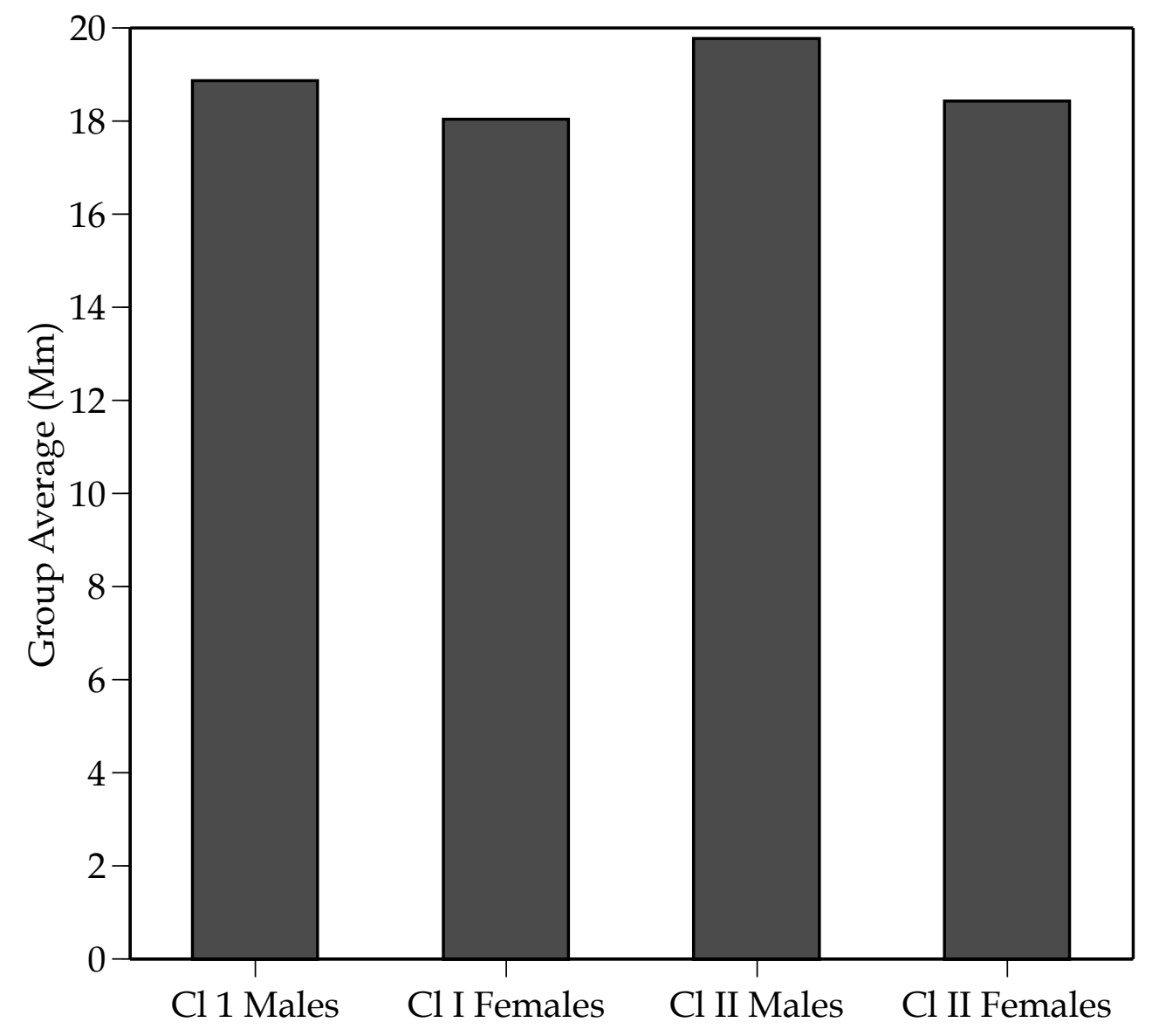

Fig. A-6. Group mean sizes, by Angle's classification and sex, for U2 pulp height. 


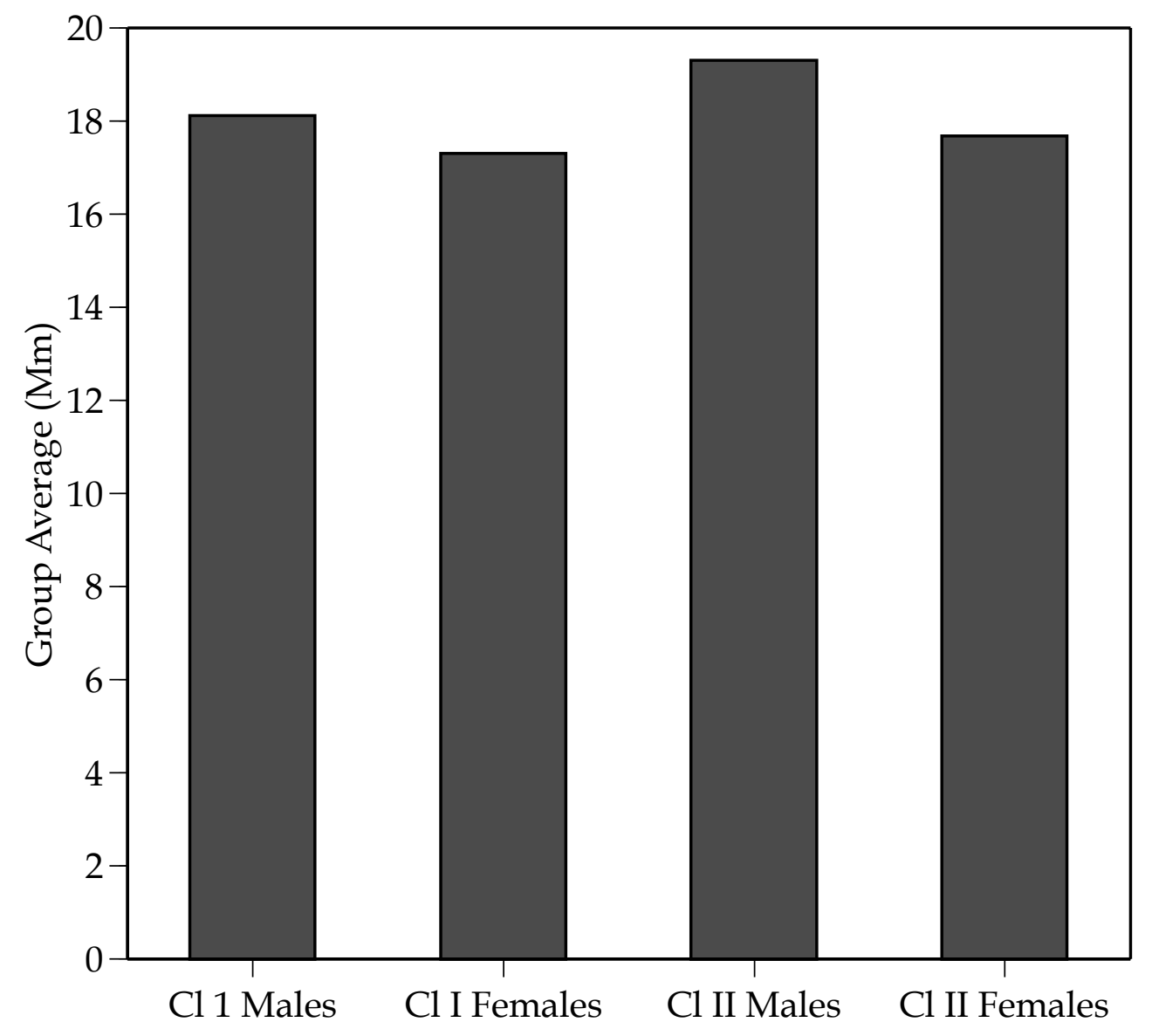

Fig. A-7. Group mean sizes, by Angle's classification and sex, for U1 mesial root length. 


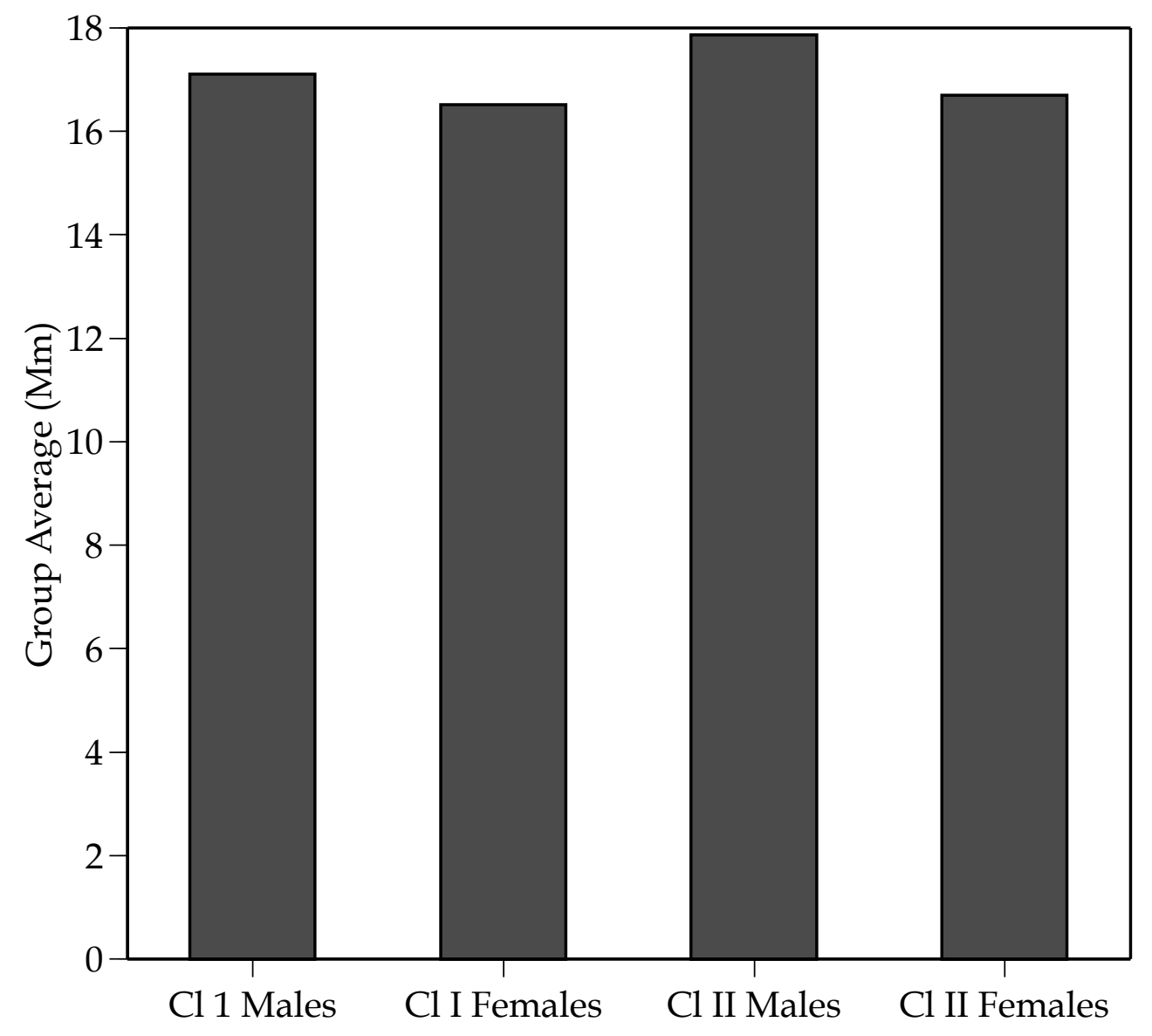

Fig. A-8. Group mean sizes, by Angle's classification and sex, for U1 distal root length. 


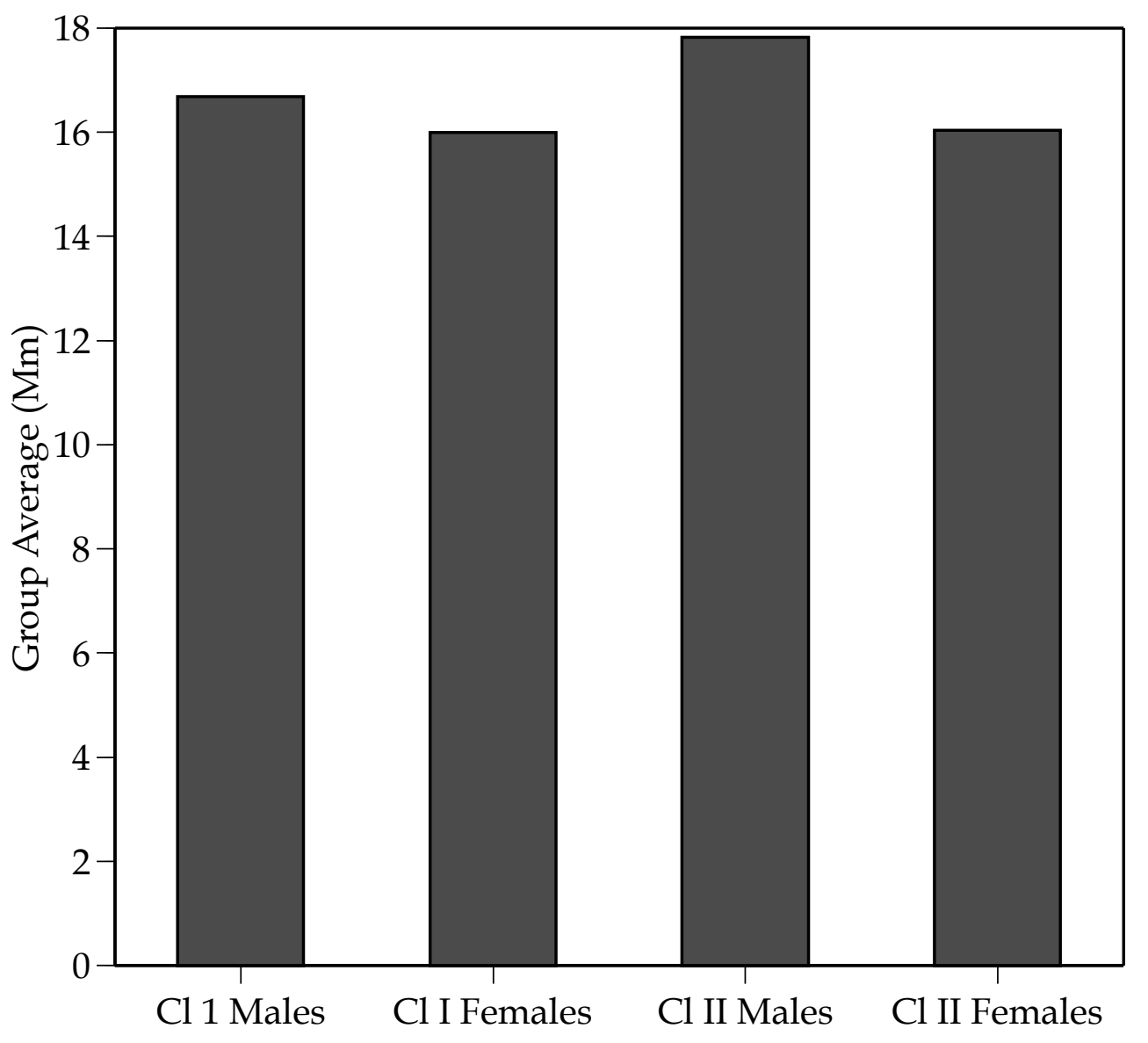

Fig. A-9. Group mean sizes, by Angle's classification and sex, for U1 mesial bone height. 


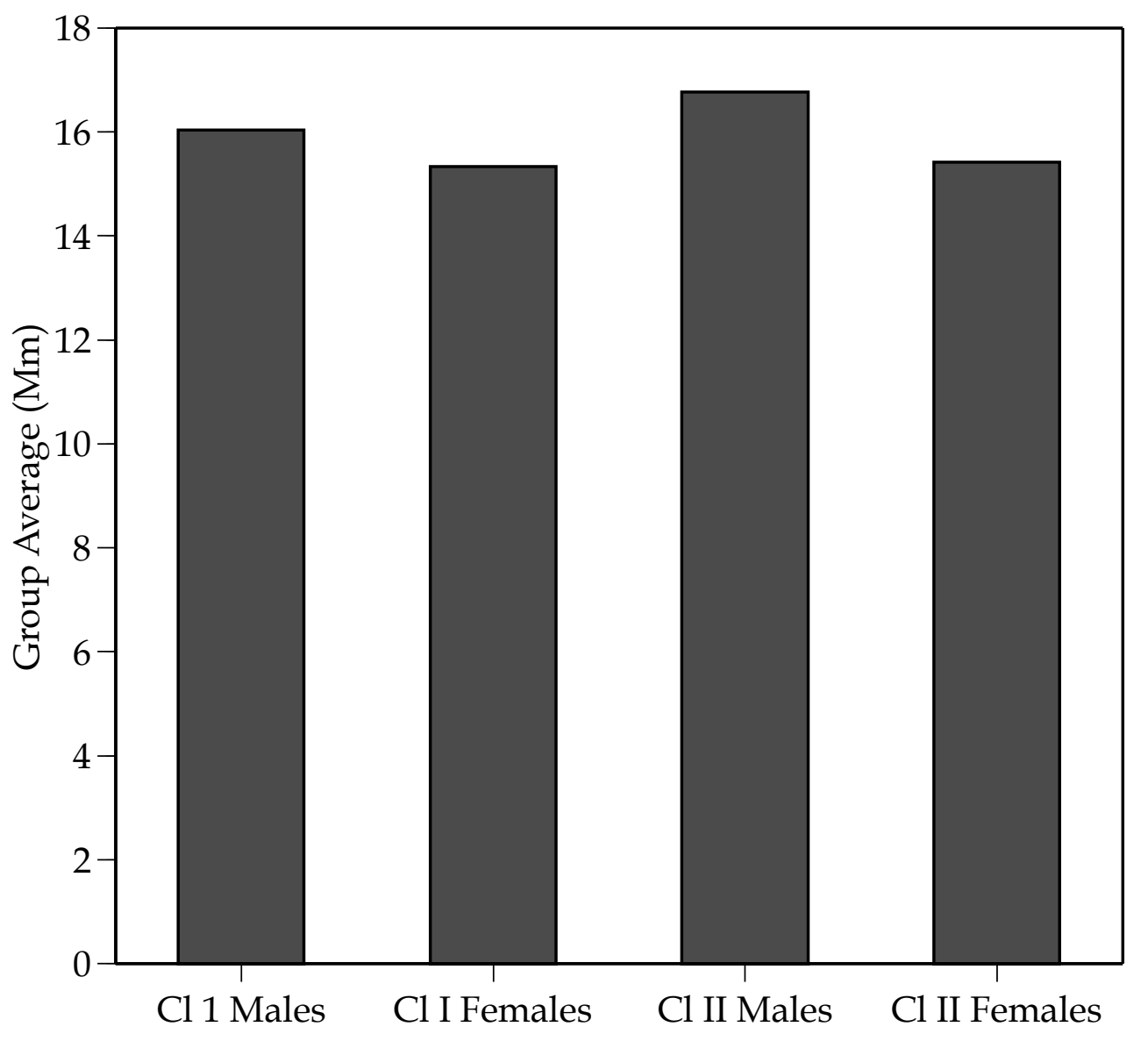

Fig. A-10. Group mean sizes, by Angle's classification and sex, for U1 distal bone height. 


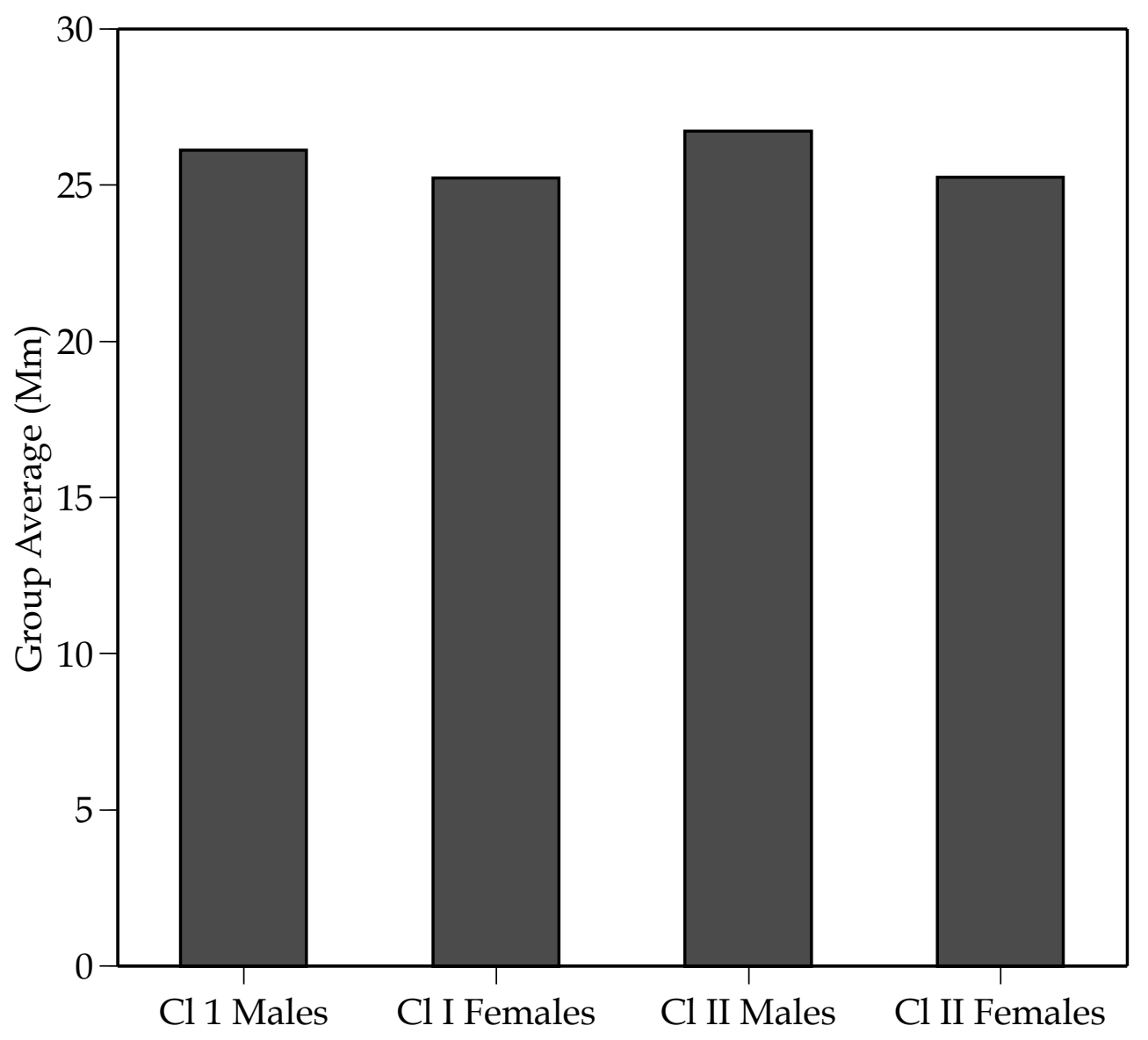

Fig. A-11. Group mean sizes, by Angle's classification and sex, for U1 tooth length. 


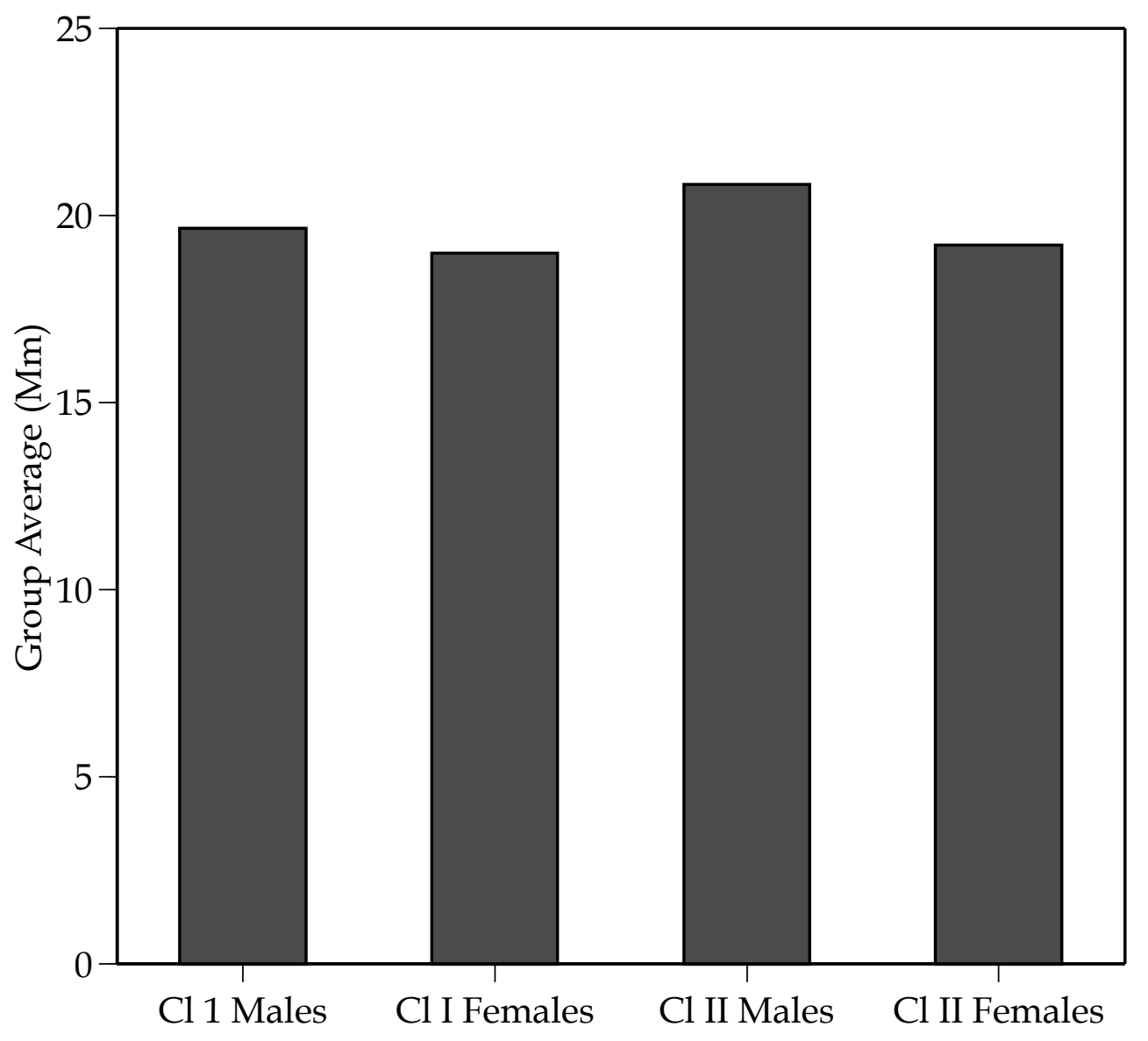

Fig. A-12. Group mean sizes, by Angle's classification and sex, for U1 pulp height. 


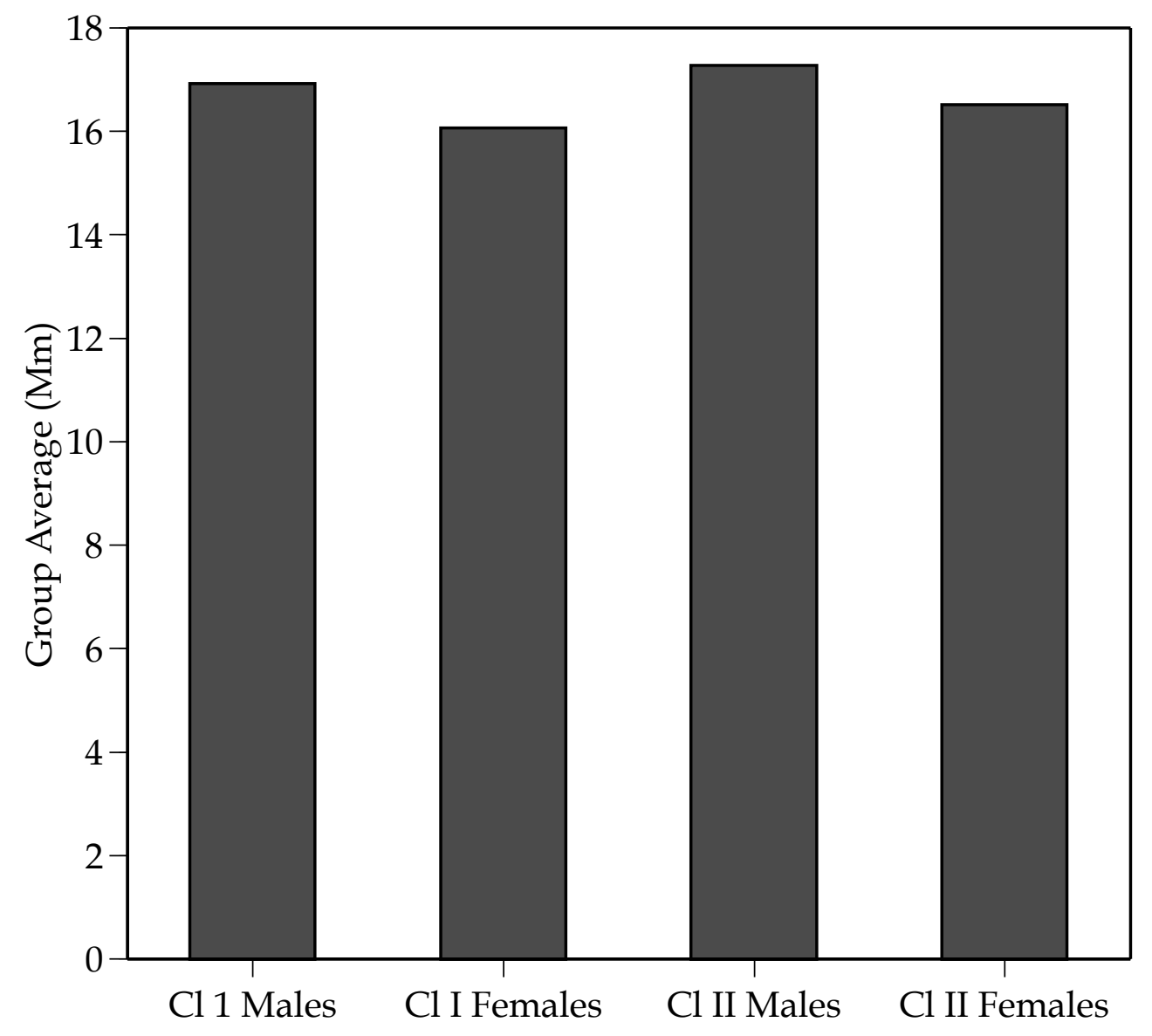

Fig. A-13. Group mean sizes, by Angle's classification and sex, for L2 mesial root length. 


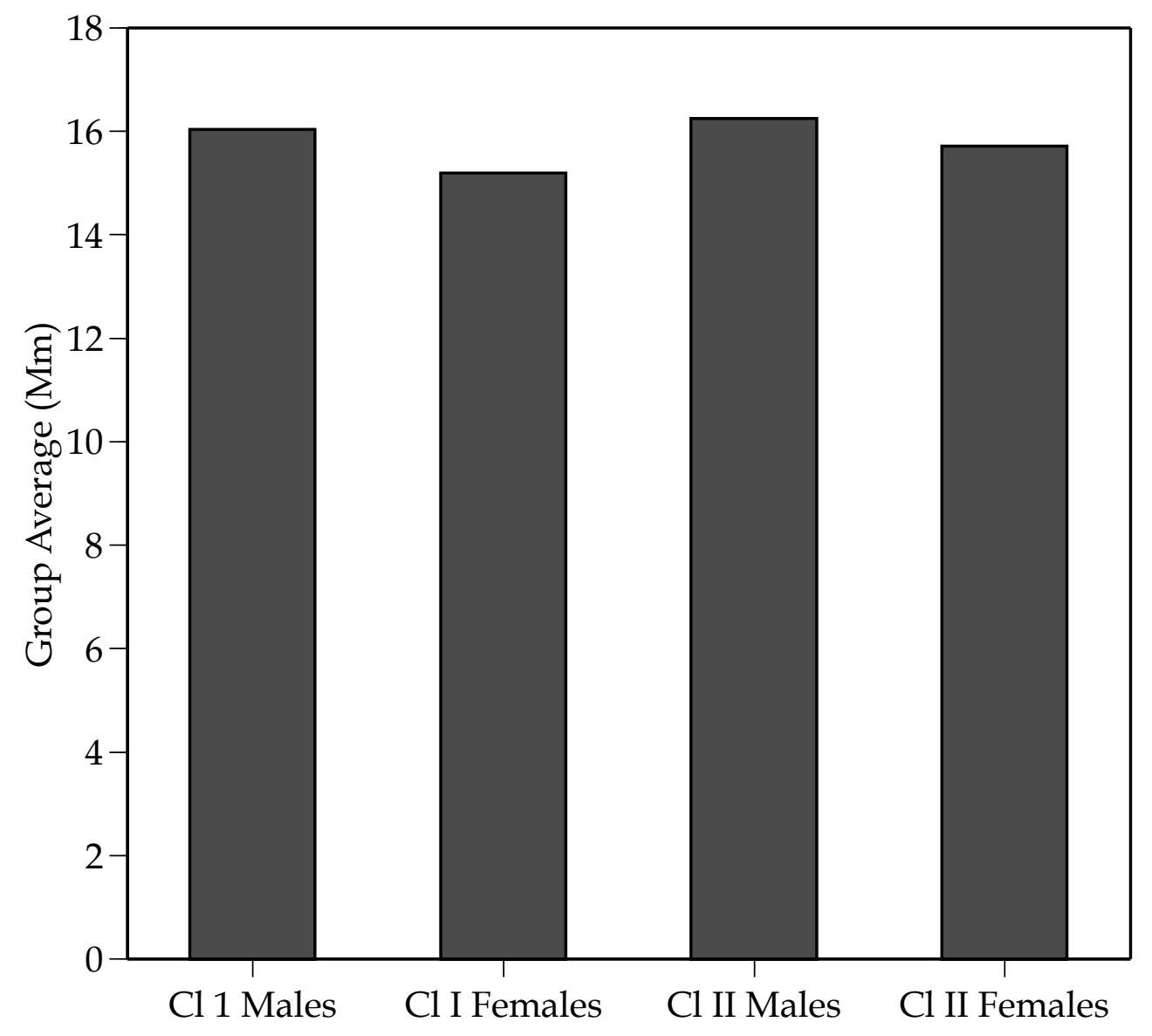

Fig. A-14. Group mean sizes, by Angle's classification and sex, for L2 distal root length. 


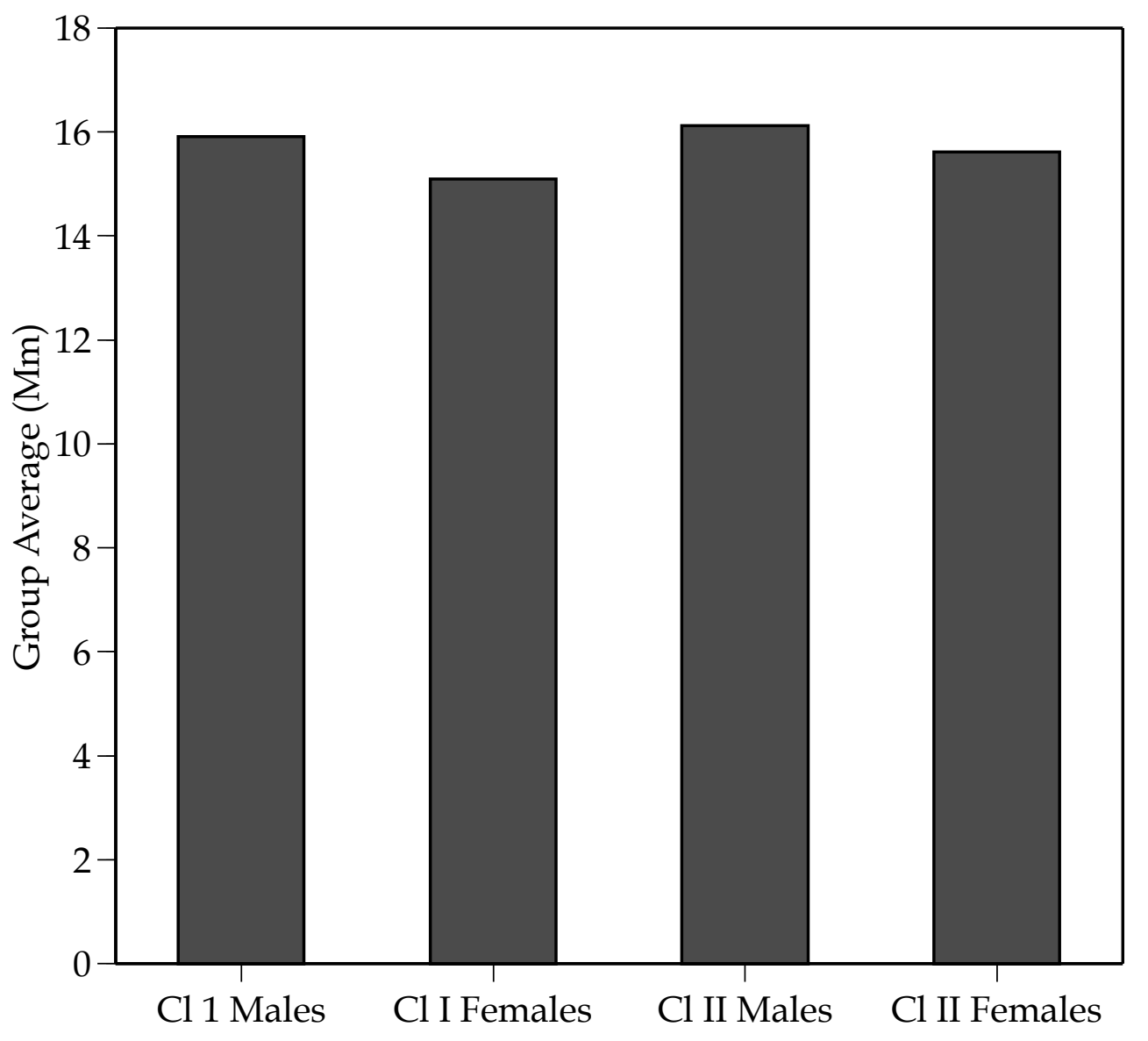

Fig. A-15. Group mean sizes, by Angle's classification and sex, for L2 mesial bone height. 


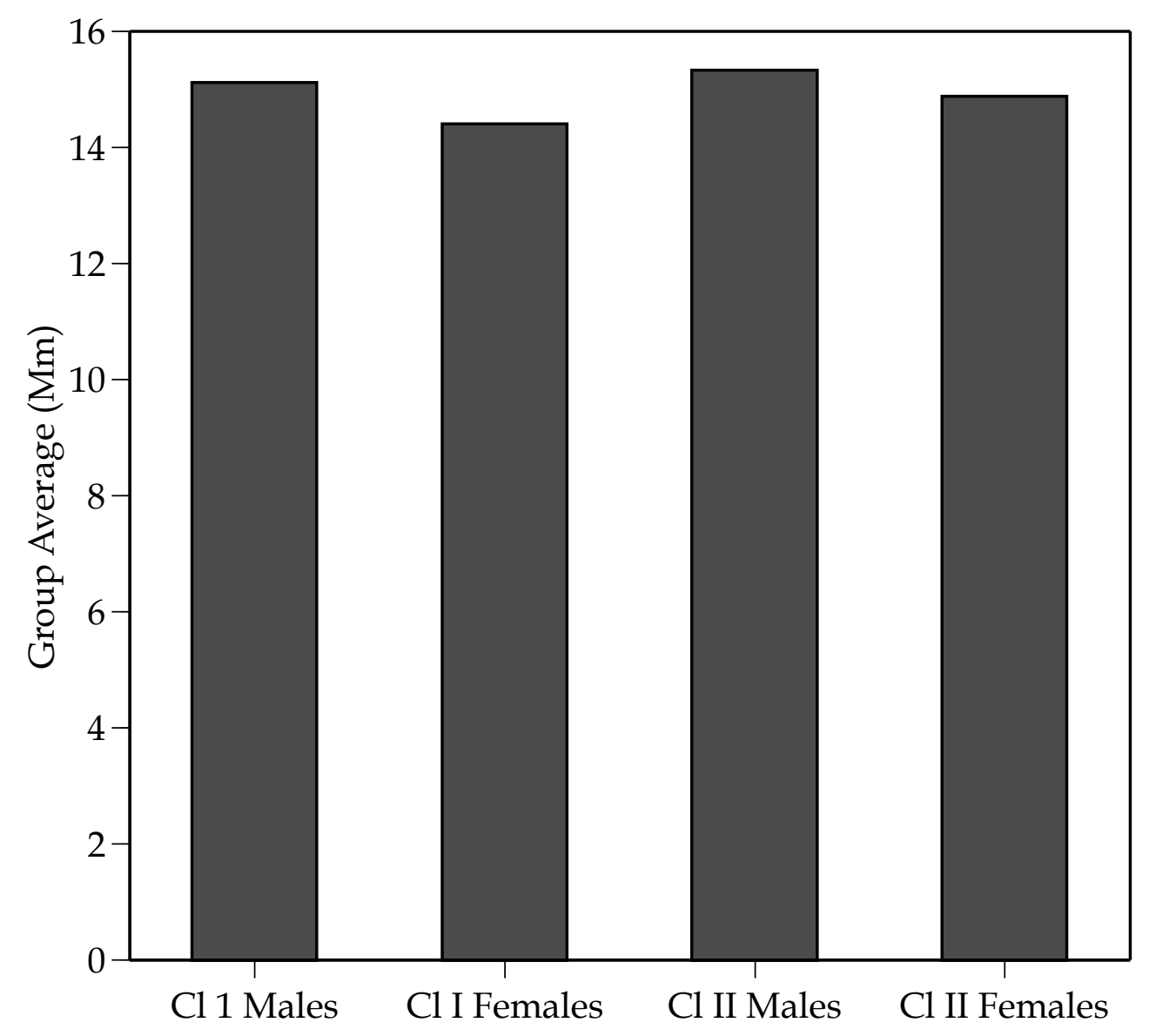

Fig. A-16. Group mean sizes, by Angle's classification and sex, for L2 distal bone height. 


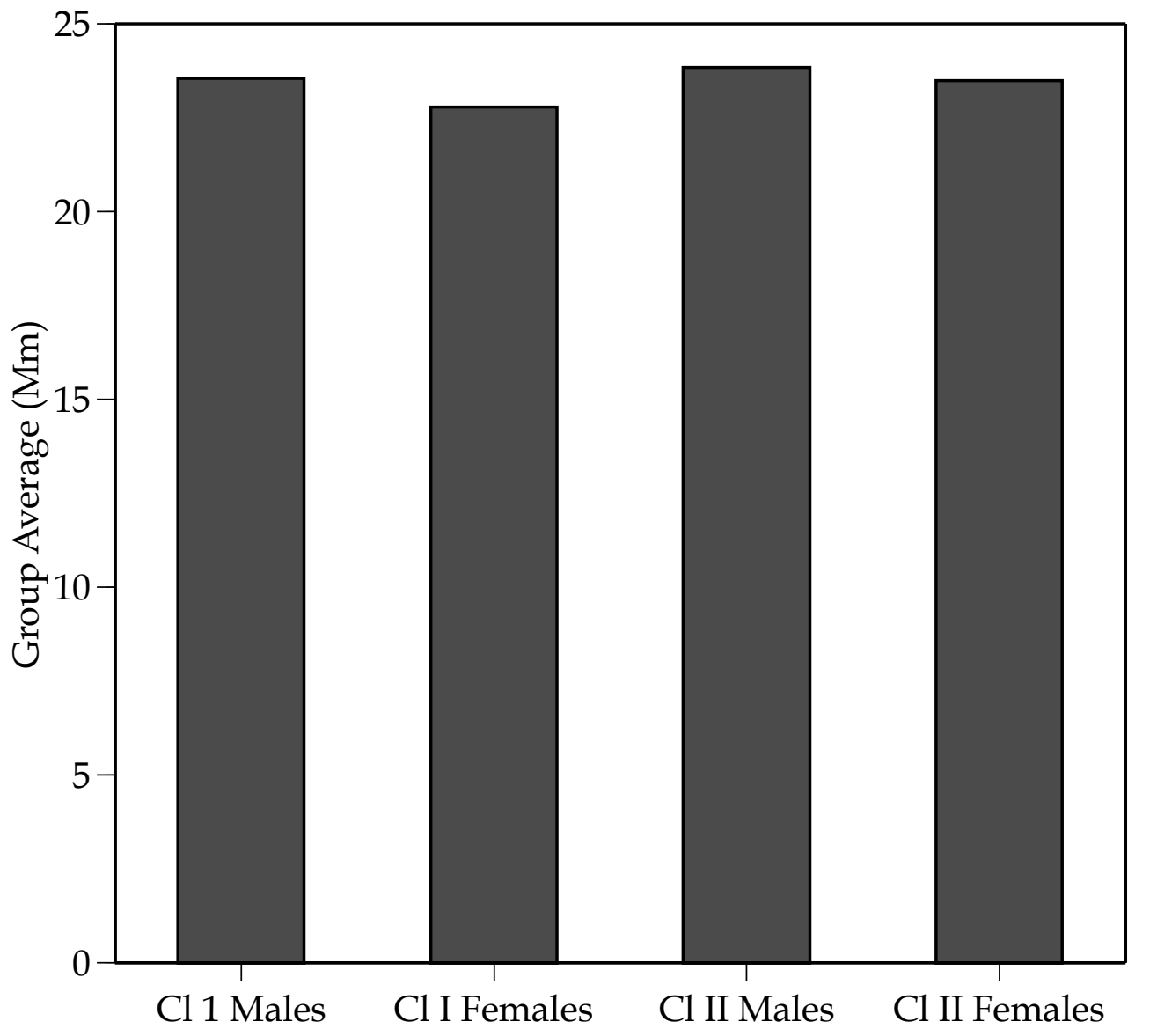

Fig. A-17. Group mean sizes, by Angle's classification and sex, for L2 tooth length. 


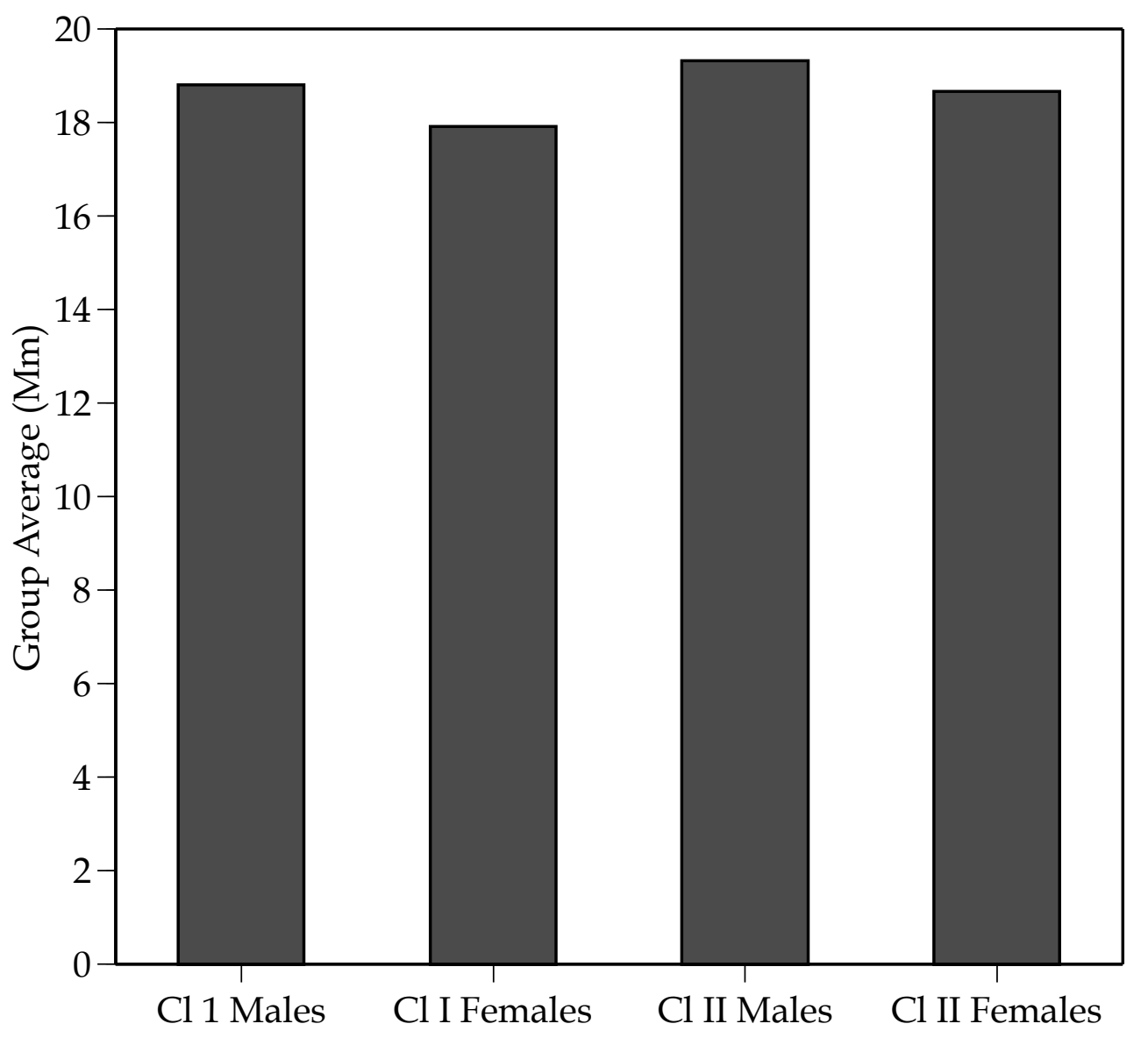

Fig. A-18. Group mean sizes, by Angle's classification and sex, for L2 pulp height. 


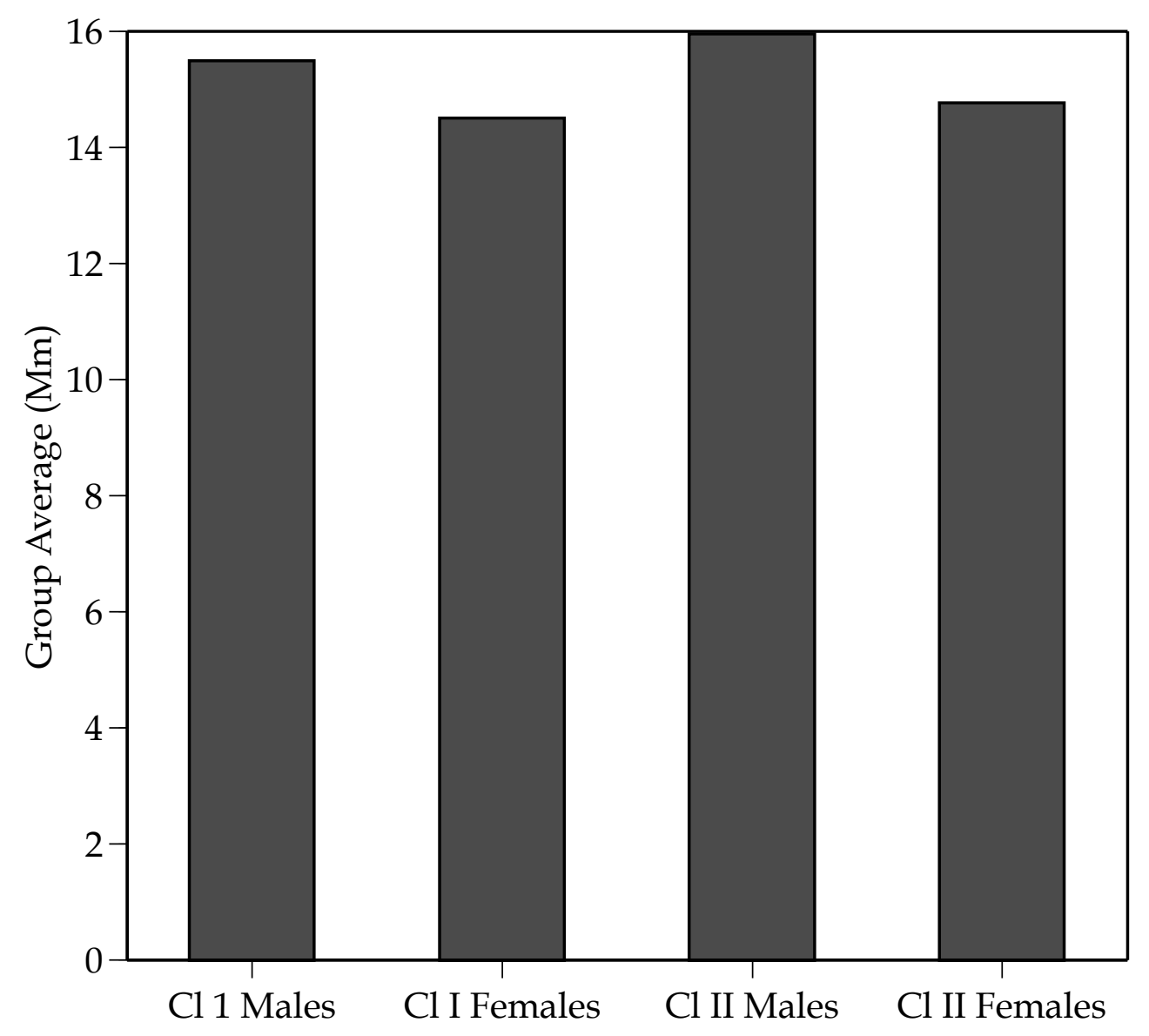

Fig. A-19. Group mean sizes, by Angle's classification and sex, for L1 mesial root length. 


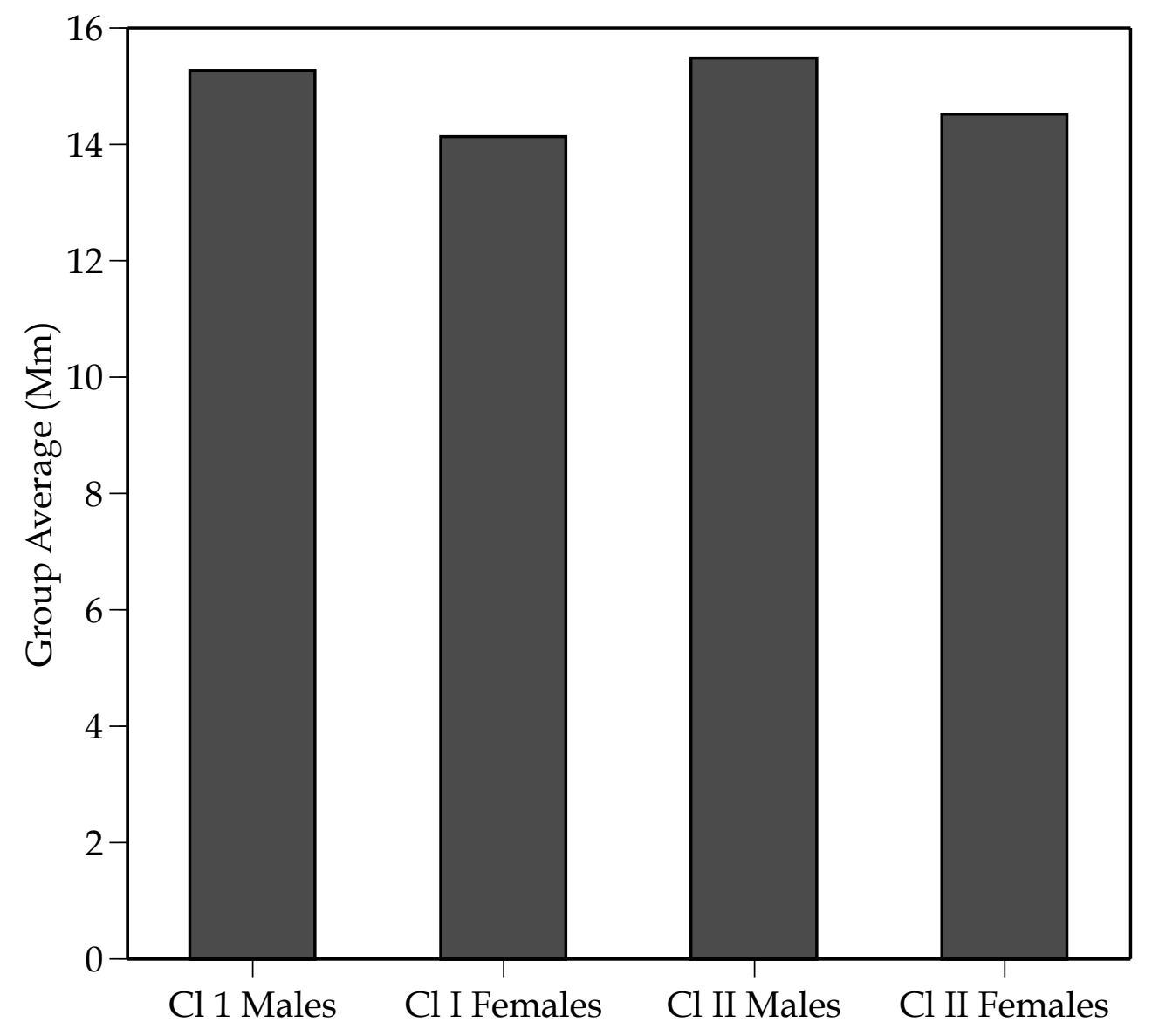

Fig. A-20. Group mean sizes, by Angle's classification and sex, for L1 distal root length. 


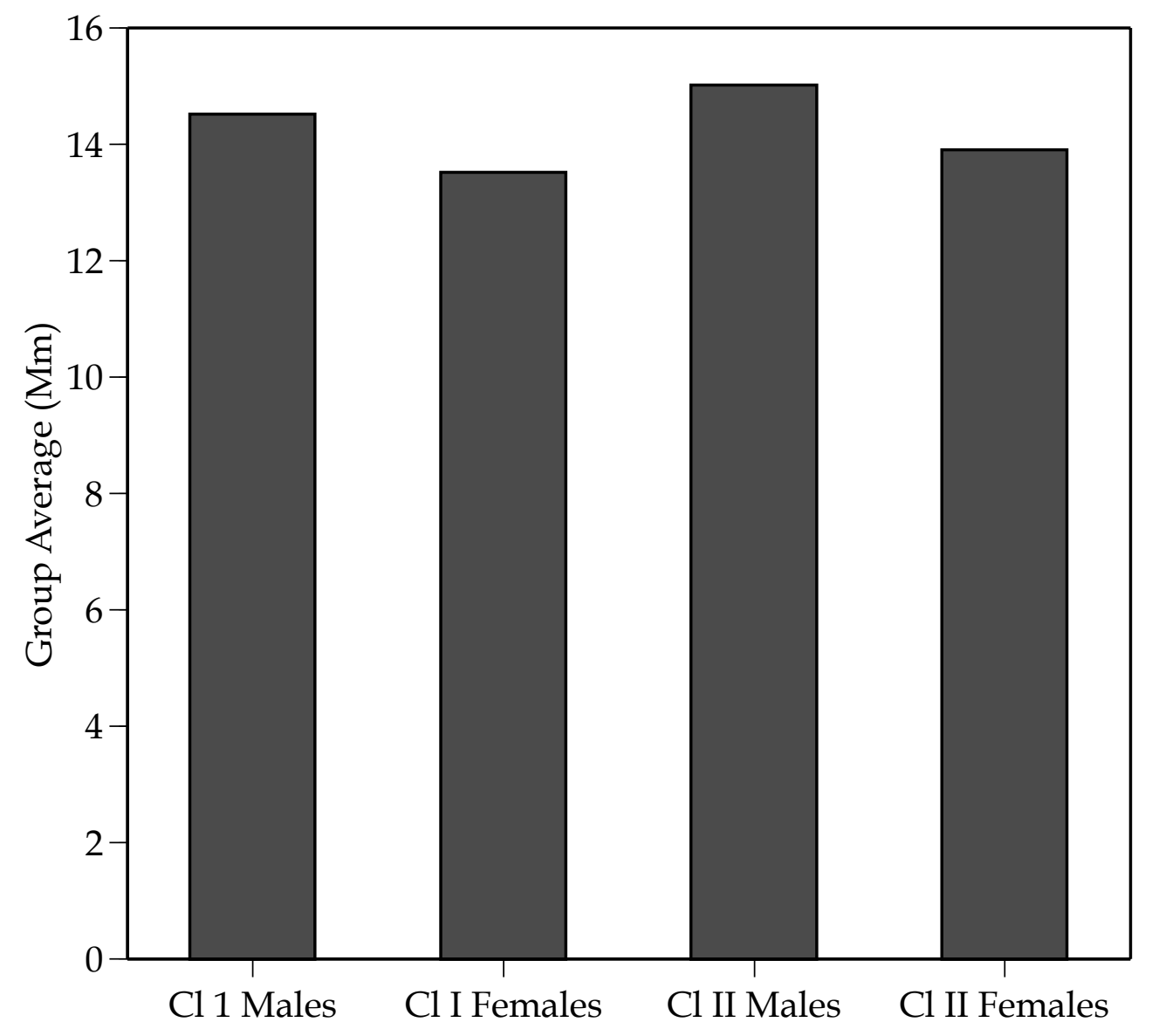

Fig. A-21. Group mean sizes, by Angle's classification and sex, for L1 mesial bone height. 


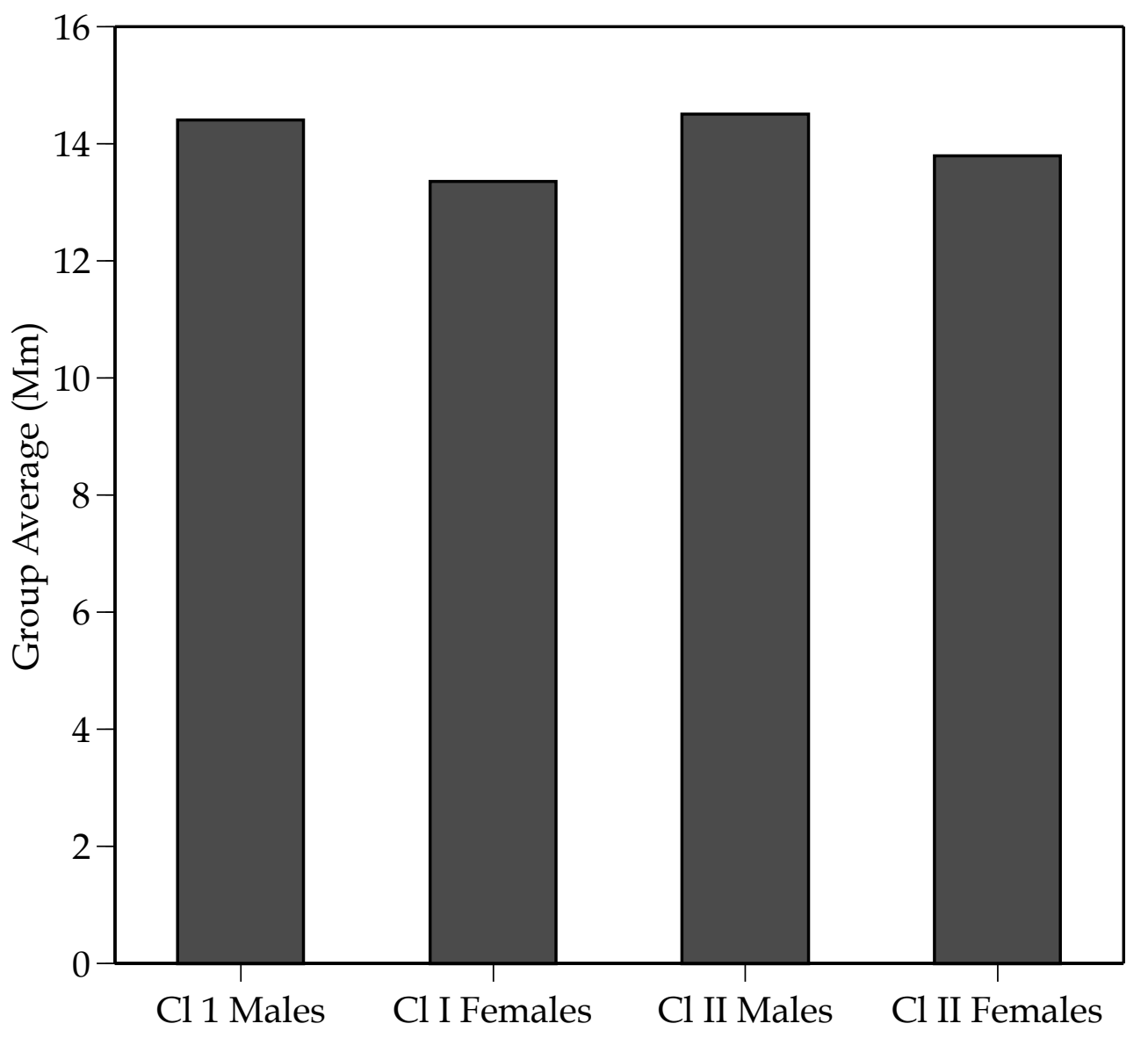

Fig. A-22. Group mean sizes, by Angle's classification and sex, for L1 distal bone height. 


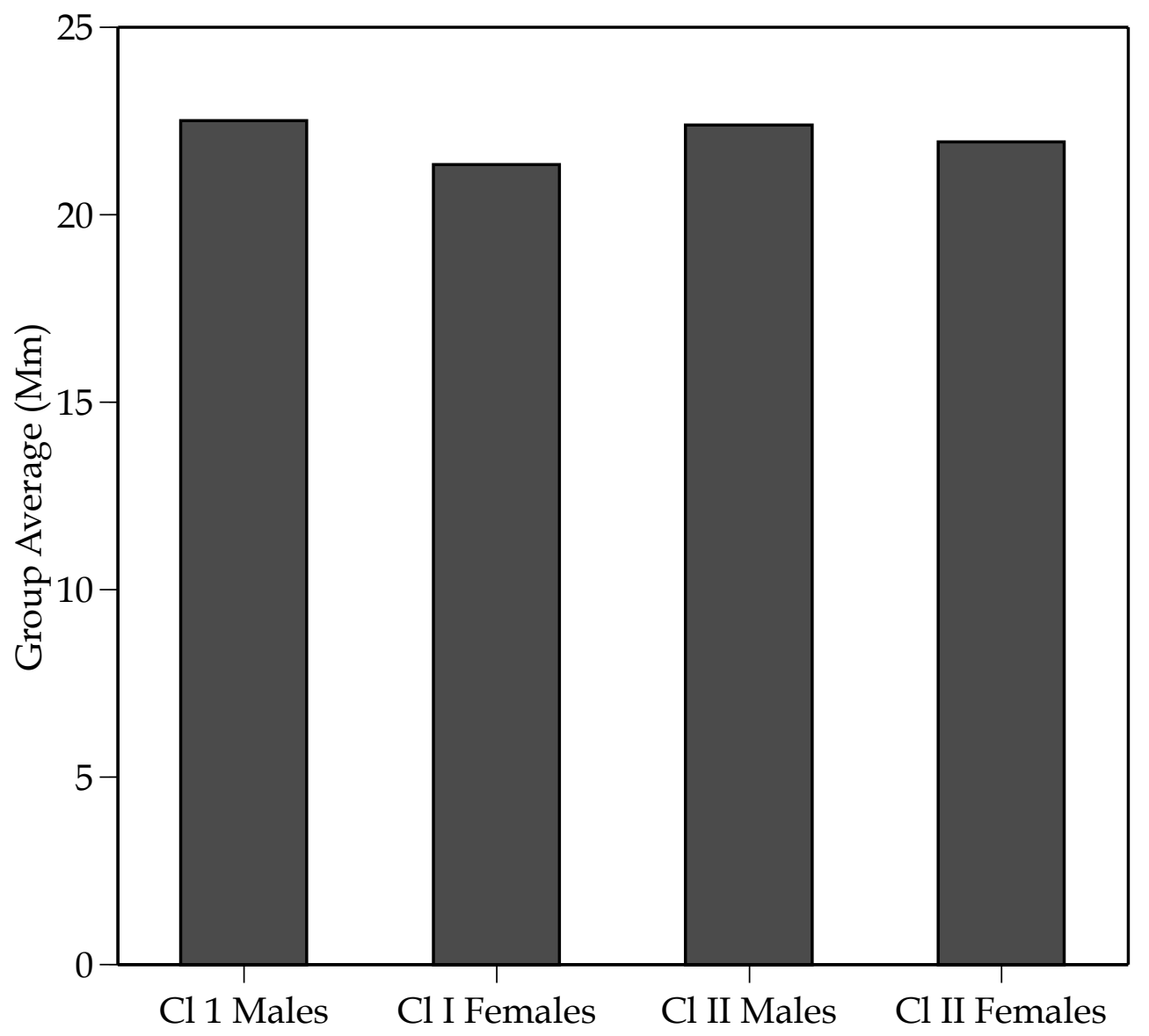

Fig. A-23. Group mean sizes, by Angle's classification and sex, for L1 tooth length. 


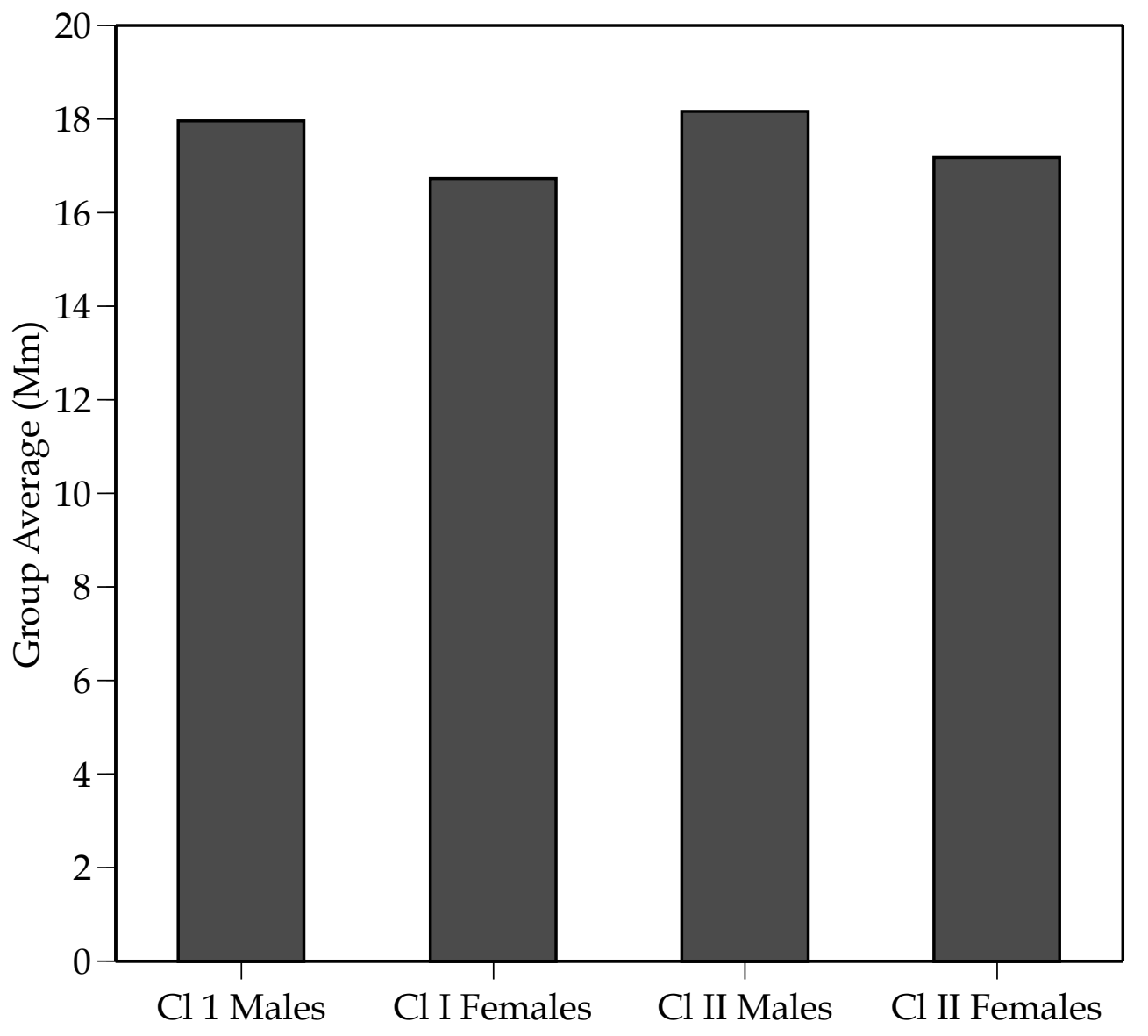

Fig. A-24. Group mean sizes, by Angle's classification and sex, for L1 pulp height. 


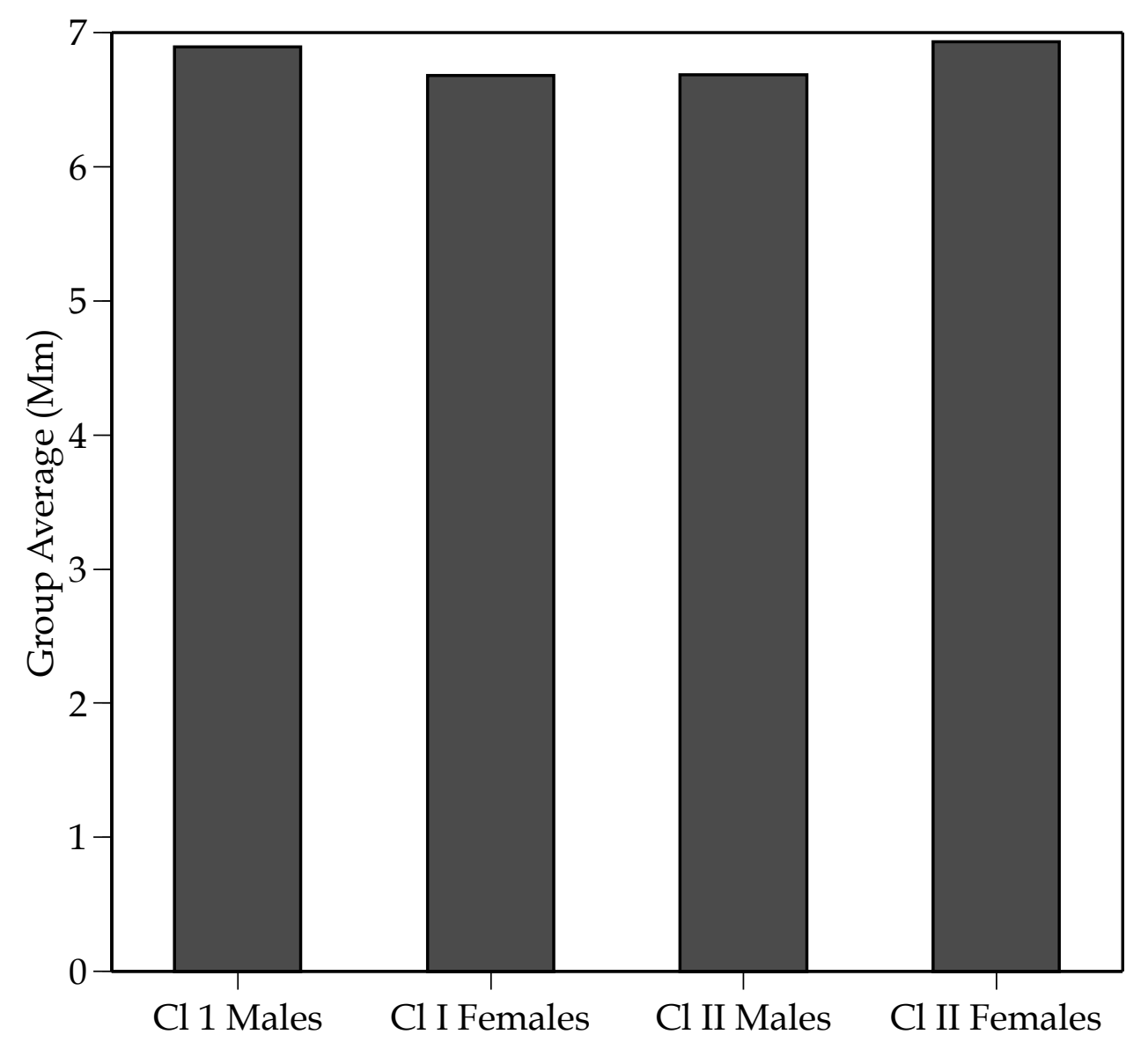

Fig. A-25. Mesiodistal mean crown diameters, by Angle's classification and sex, for maxillary right lateral incisor. 


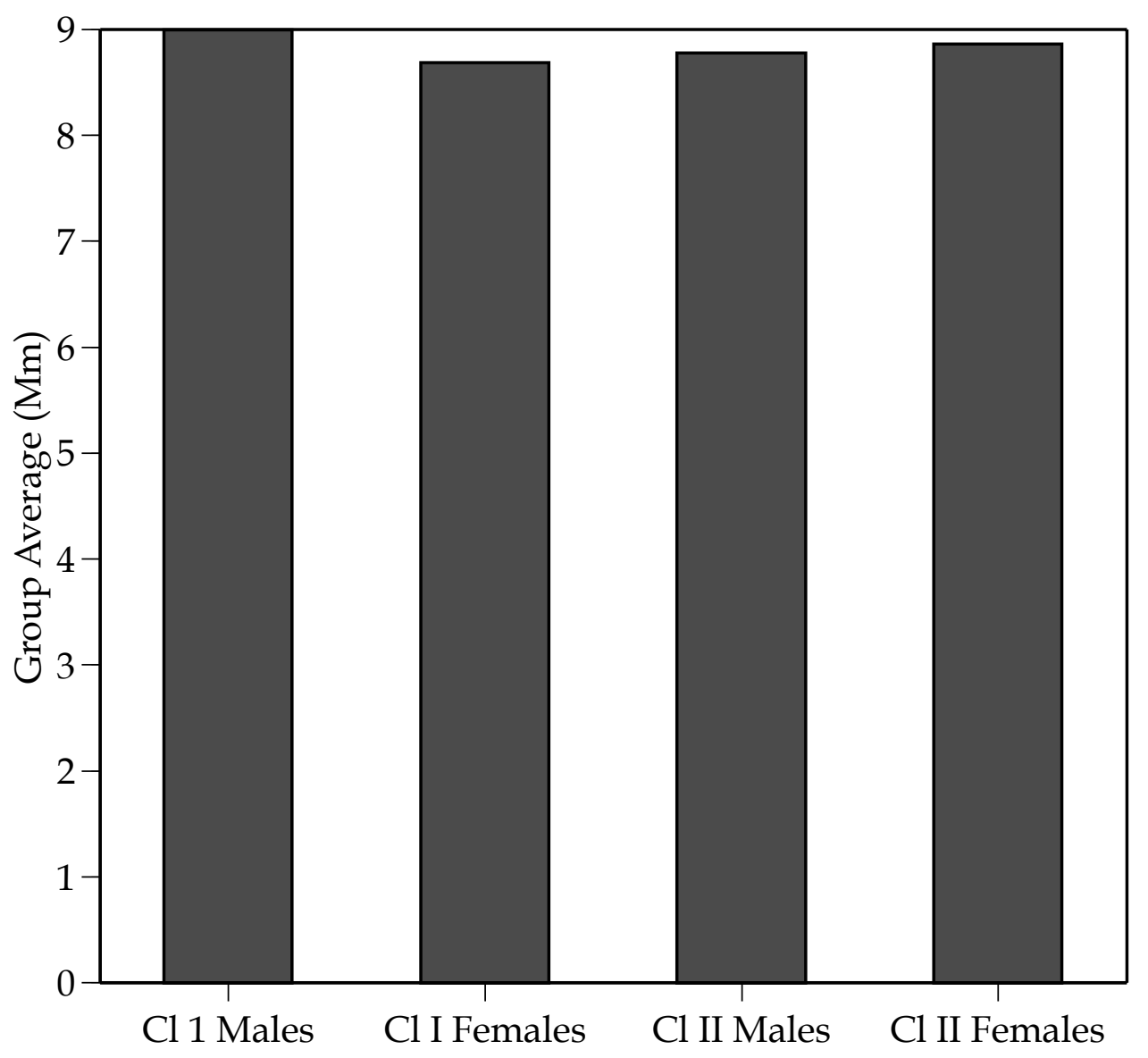

Fig. A-26. Mesiodistal mean crown diameters, by Angle's classification and sex, for maxillary right central incisor. 


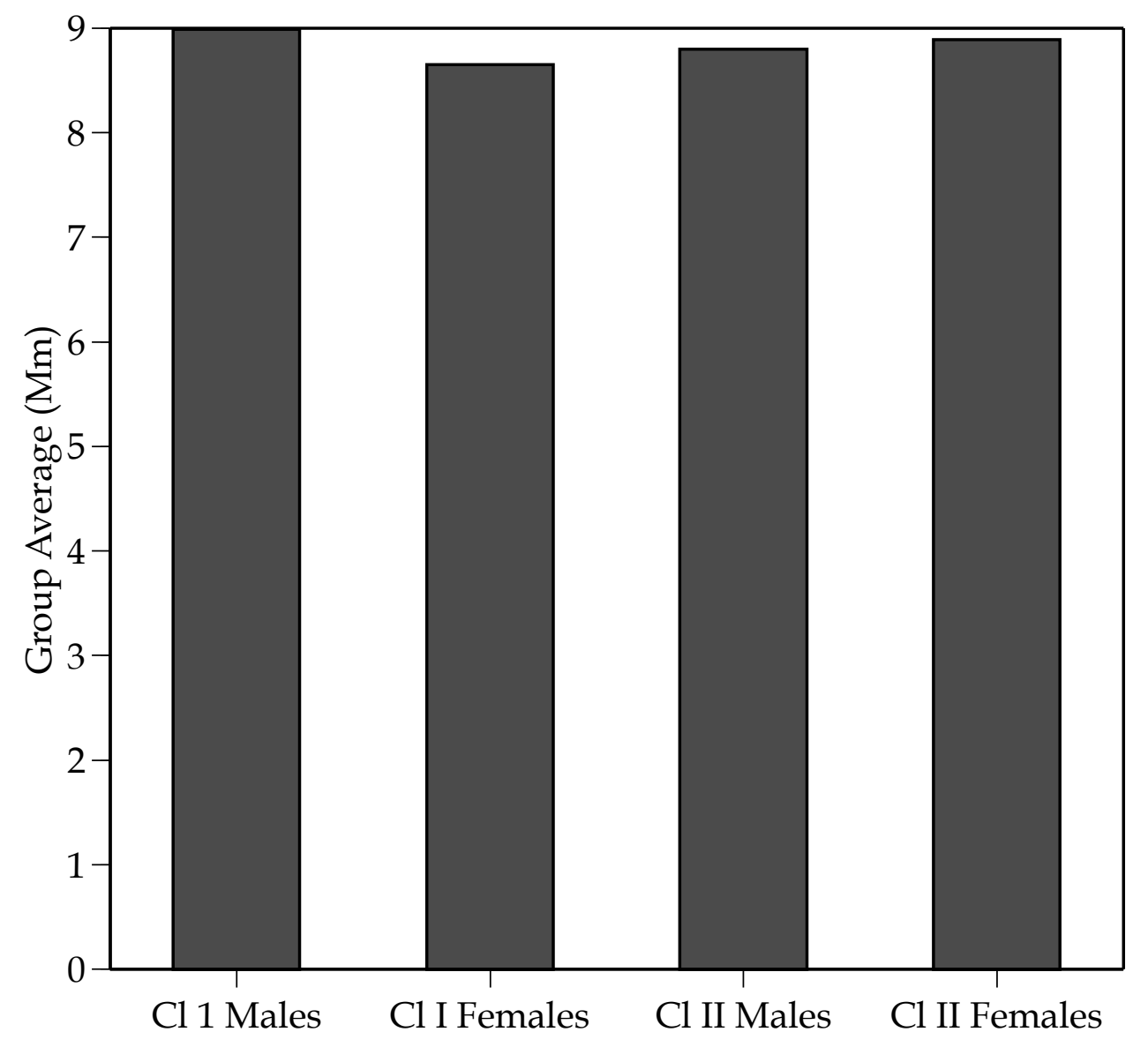

Fig. A-27. Mesiodistal mean crown diameters, by Angle's classification and sex, for maxillary left central incisor. 


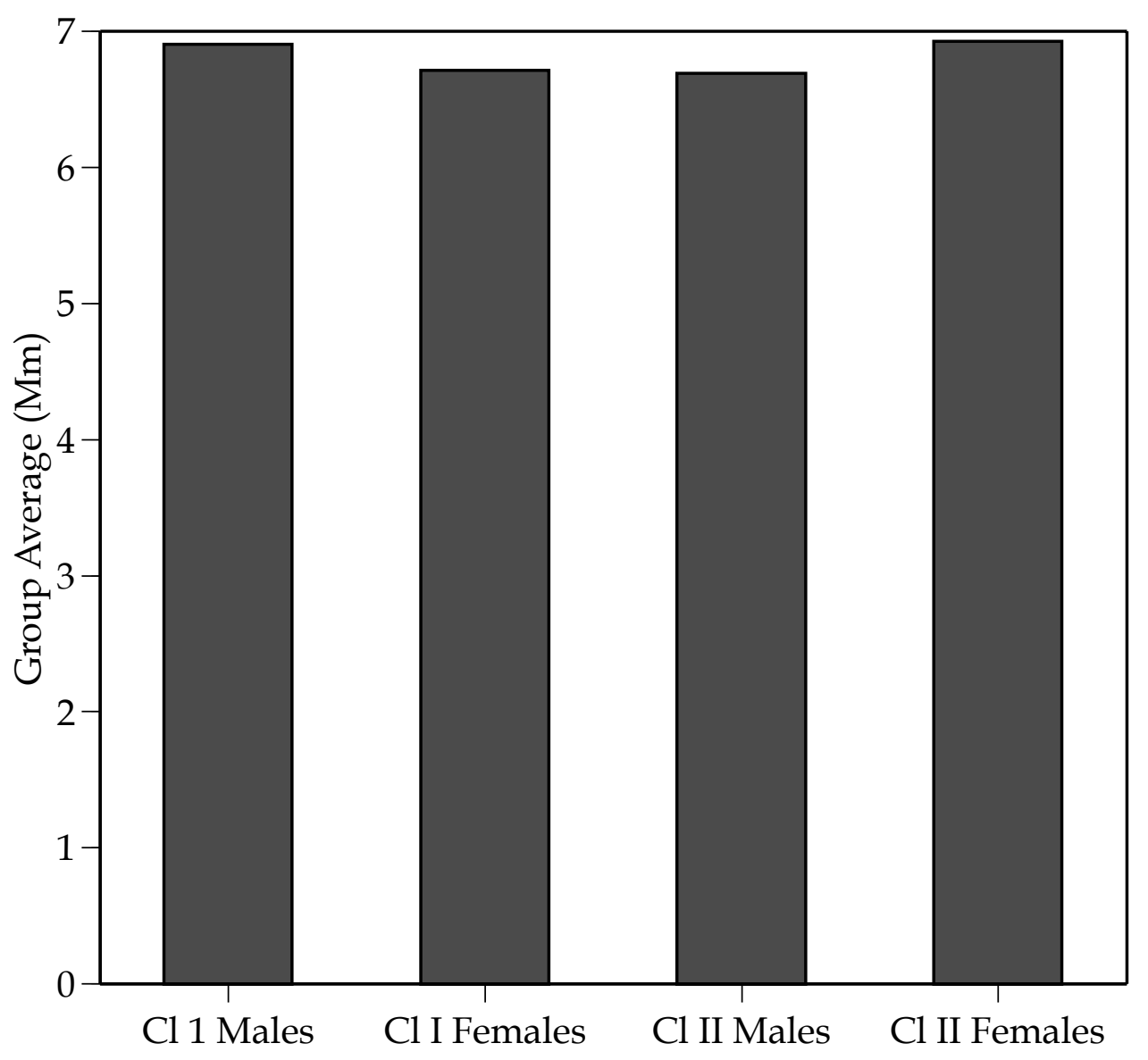

Fig. A-28. Mesiodistal mean crown diameters, by Angle's classification and sex, for maxillary left lateral incisor. 


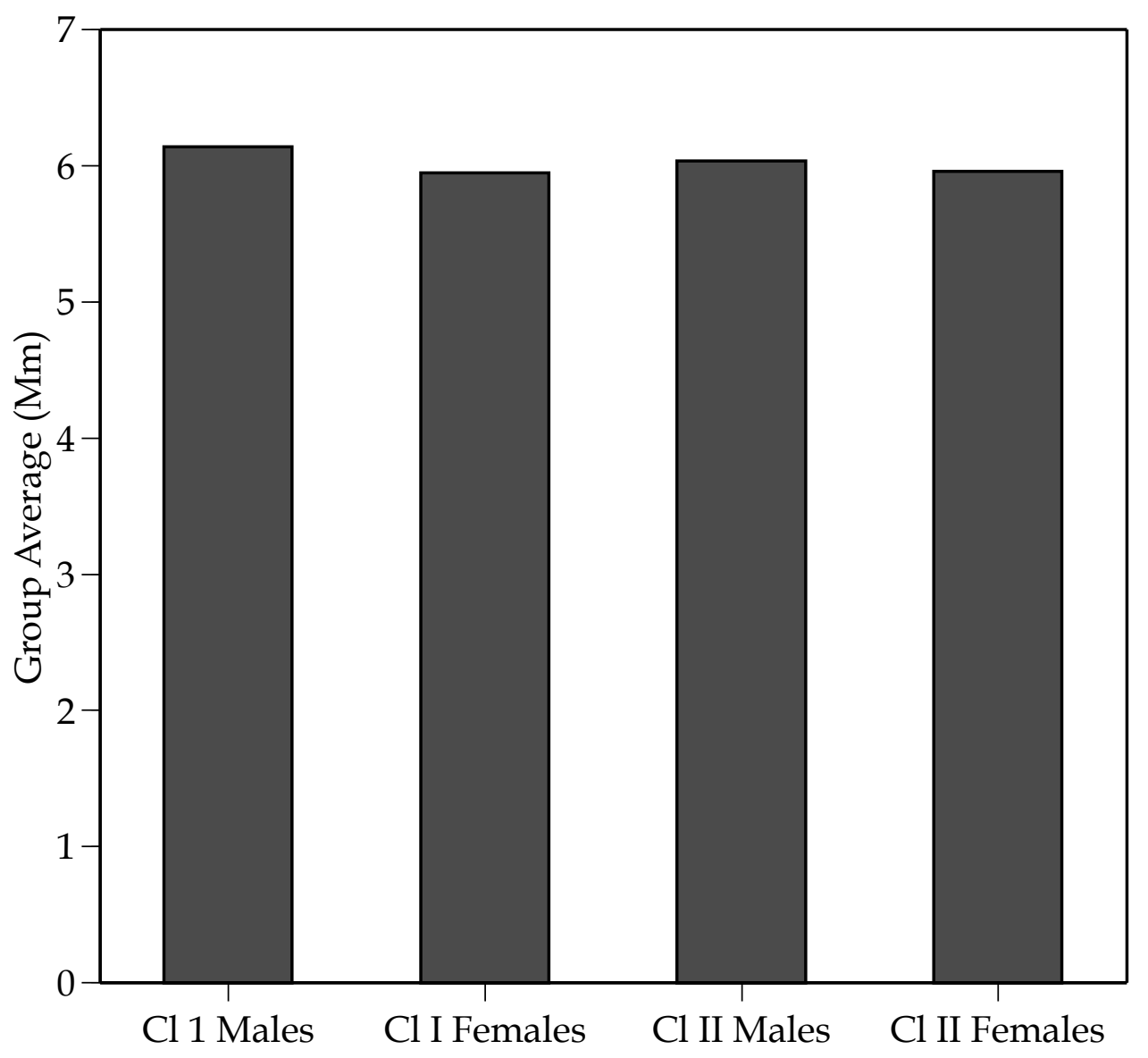

Fig. A-29. Mesiodistal mean crown diameters, by Angle's classification and sex, for mandibular left lateral incisor. 


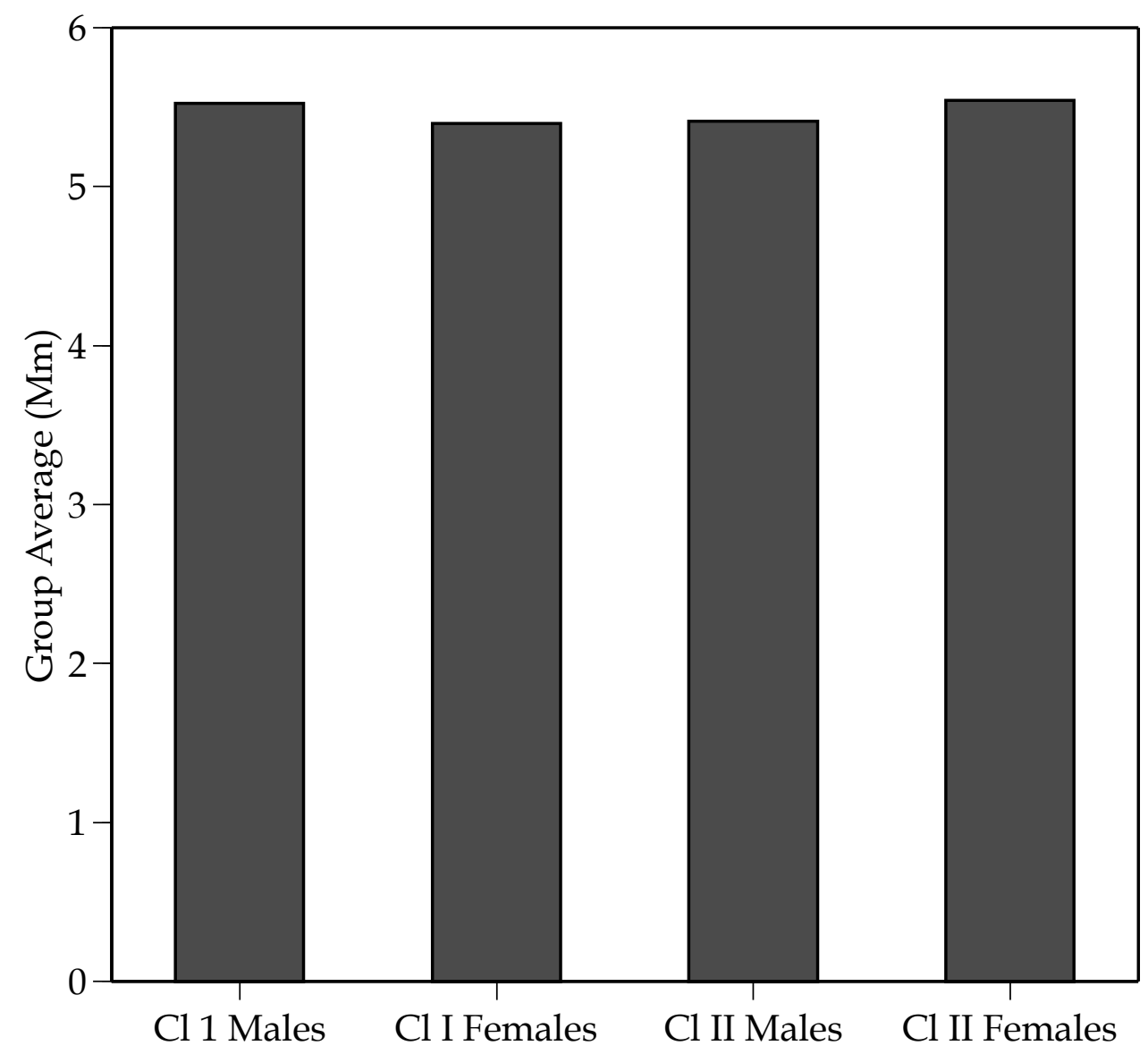

Fig. A-30. Mesiodistal mean crown diameters, by Angle's classification and sex, for mandibular left central incisor. 


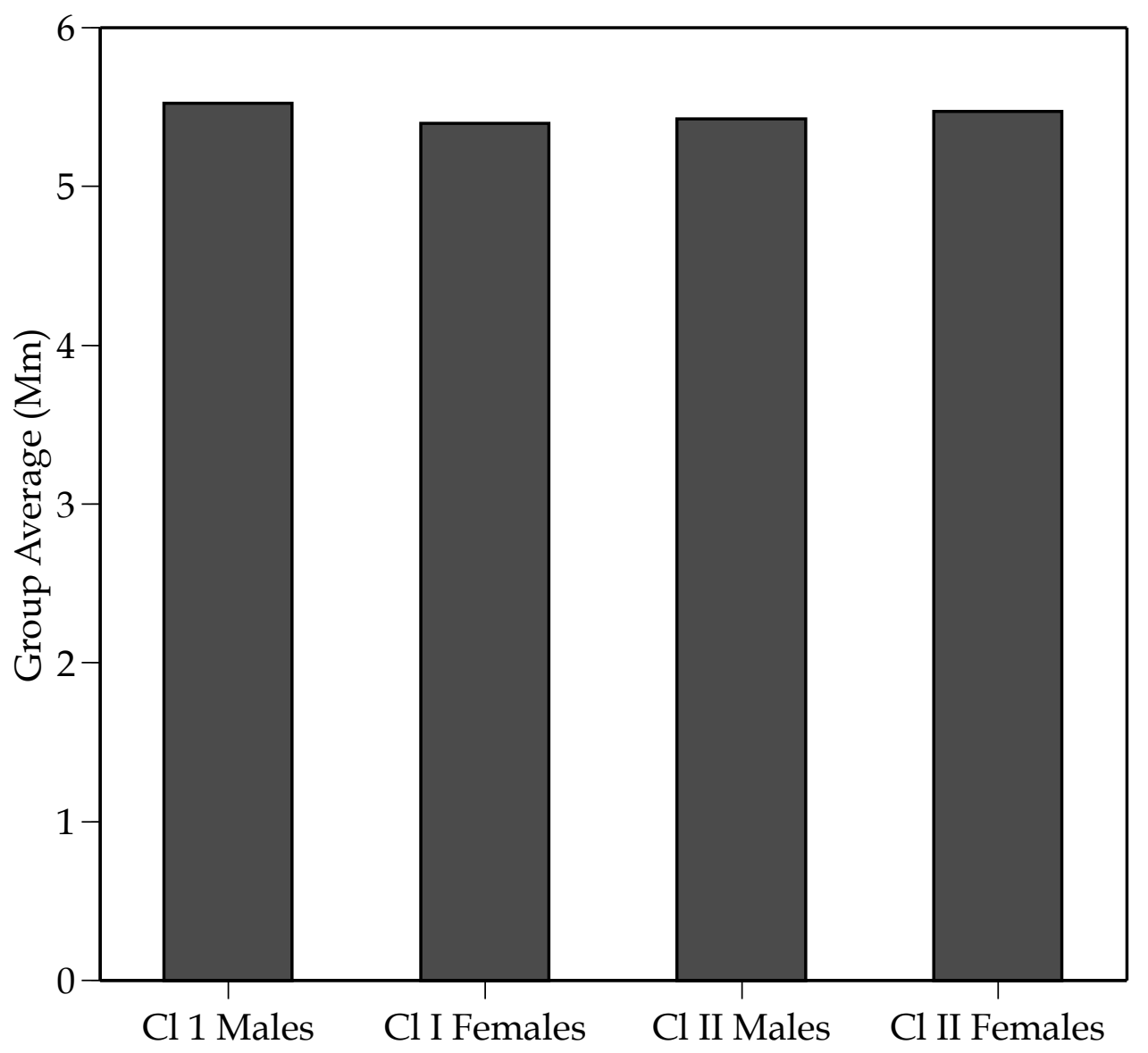

Fig. A-31. Mesiodistal mean crown diameters, by Angle's classification and sex, for mandibular right central incisor. 


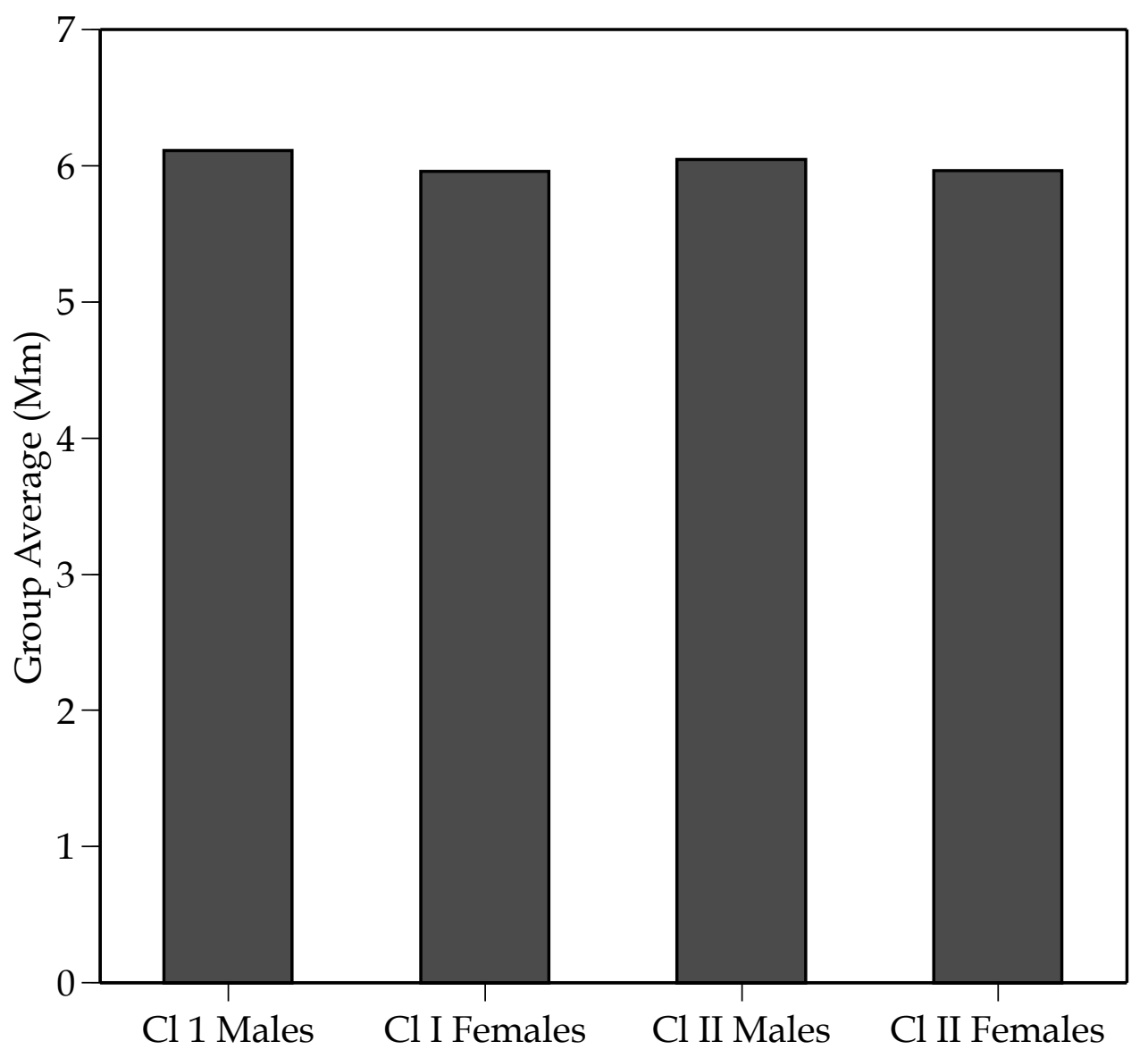

Fig. A-32. Mesiodistal mean crown diameters, by Angle's classification and sex, for mandibular right lateral incisor. 


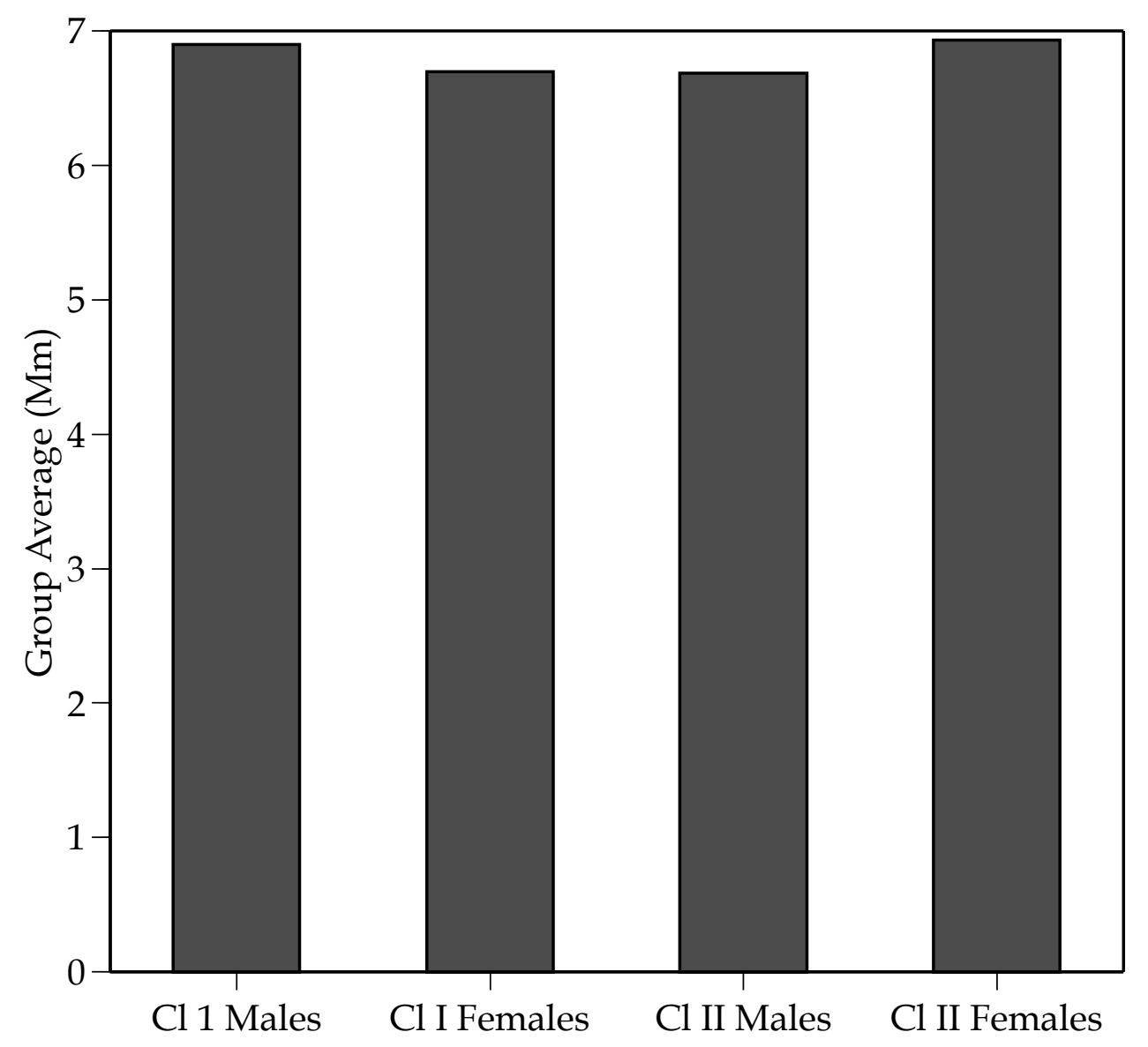

Fig. A-33. Mesiodistal mean crown diameters, by Angle's classification and sex, for maxillary lateral incisor averaged across sides. 


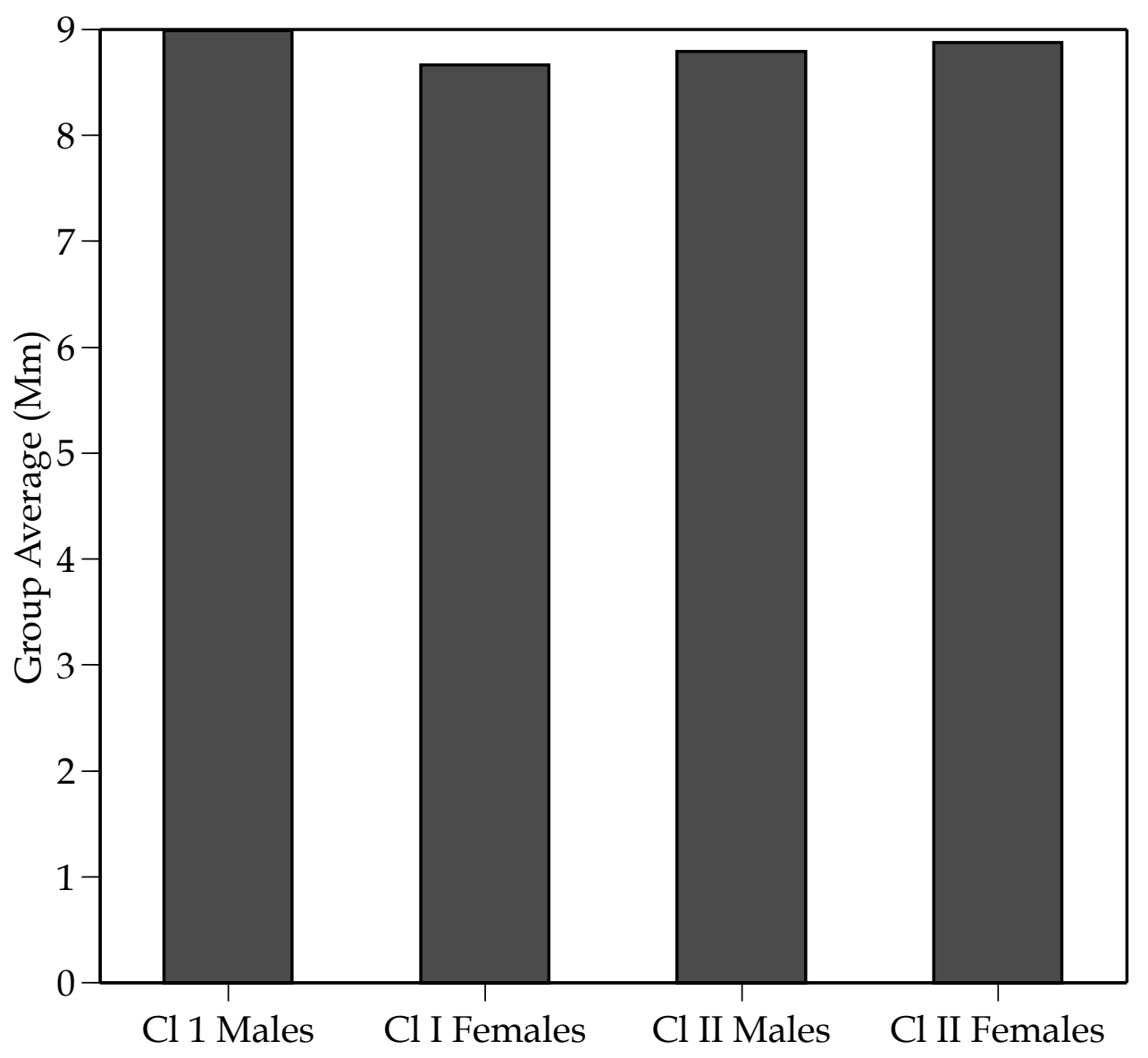

Fig. A-34. Mesiodistal mean crown diameters, by Angle's classification and sex, for maxillary central incisor averaged across sides. 


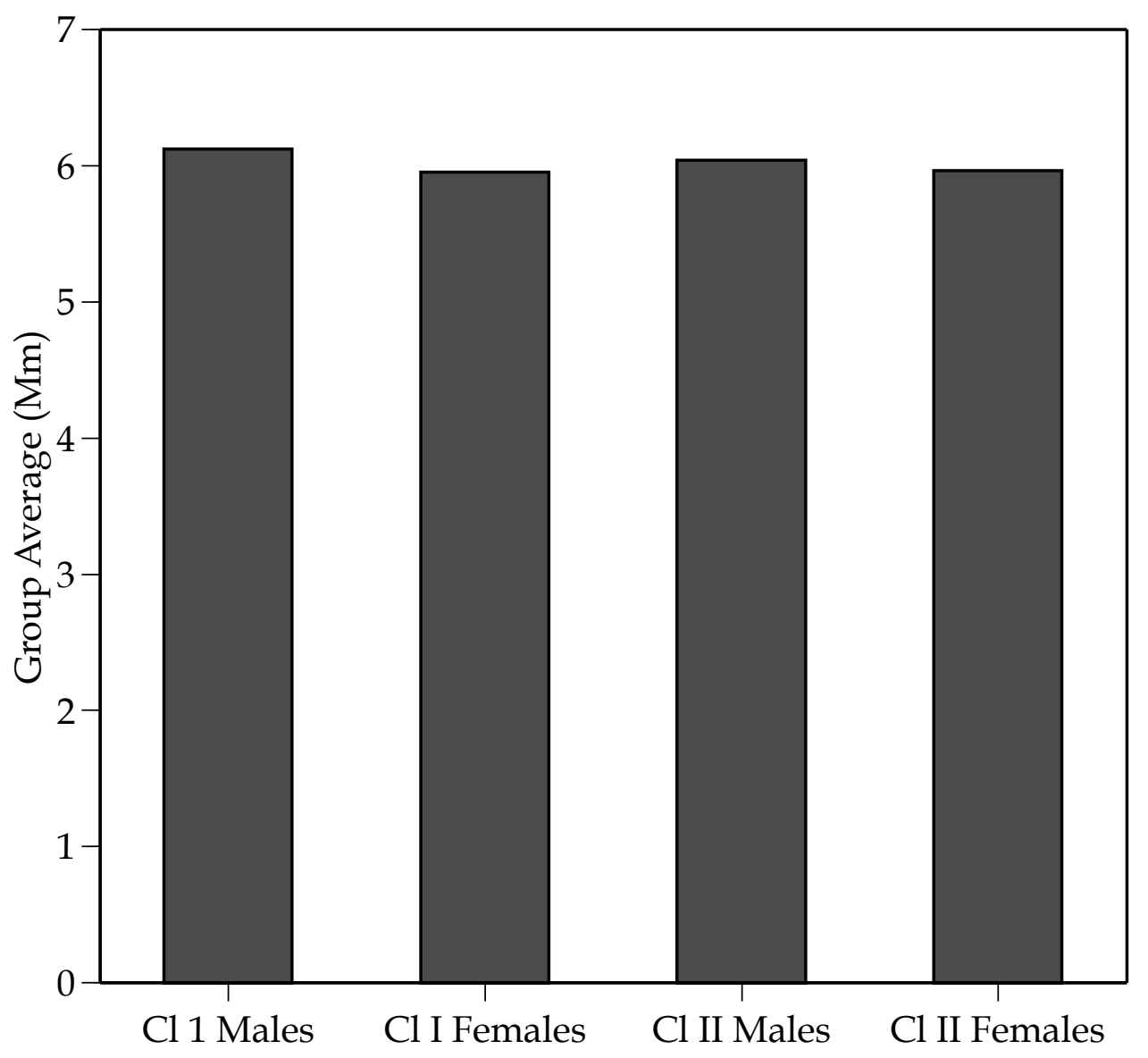

Fig. A-35. Mesiodistal mean crown diameters, by Angle's classification and sex, for mandibular lateral incisor averaged across sides. 


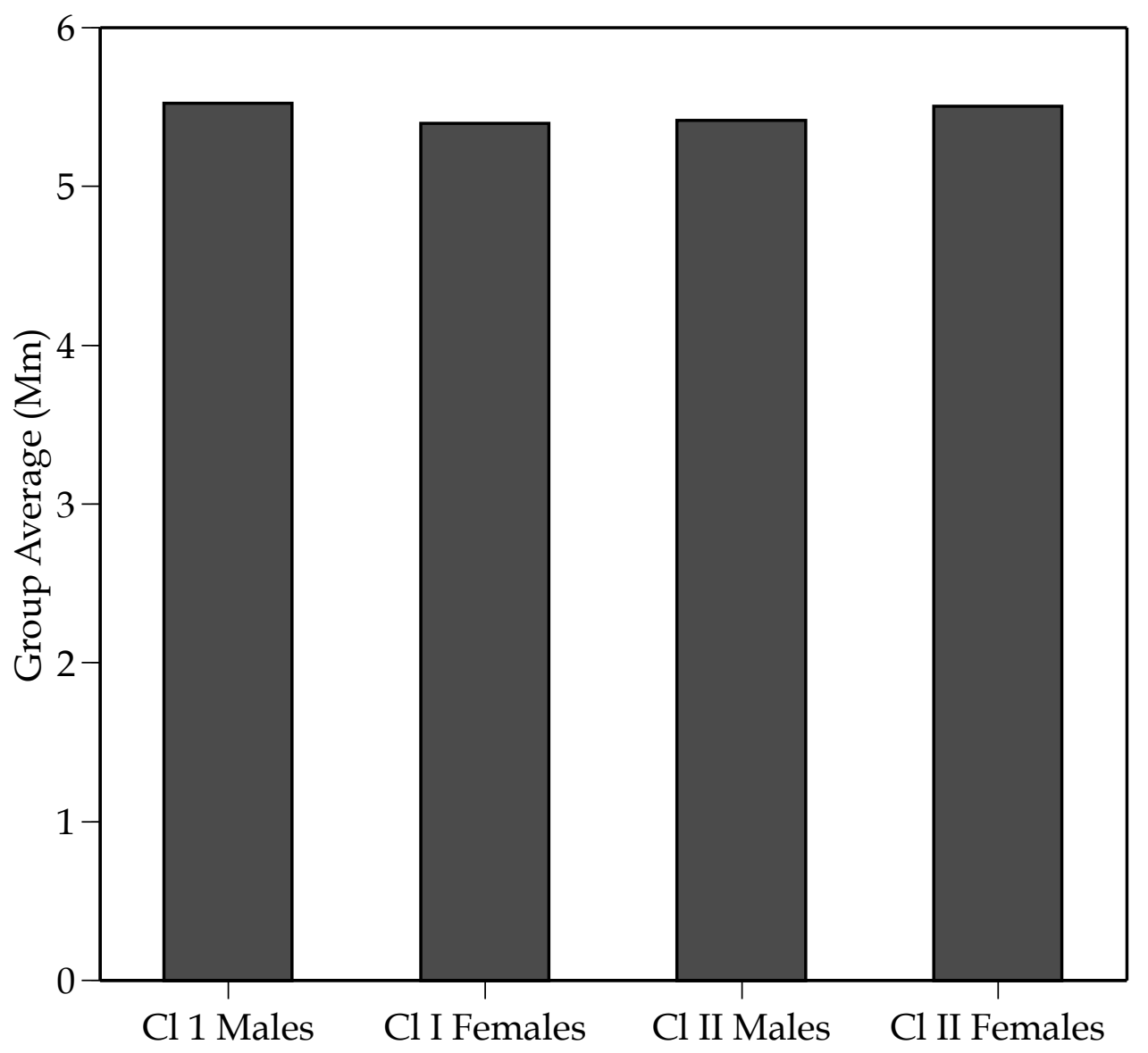

Fig. A-36. Mesiodistal mean crown diameters, by Angle's classification and sex, for mandibular central incisor averaged across sides. 
William Max Couch, Jr. was born in Fayetteville, North Carolina, on May 2, 1973. He attended elementary school and graduated from Flint River Academy in May, 1991 in Woodbury, Georgia. The following month he entered The University of Georgia, Athens and in March, 1994 received early acceptance to The University of Tennessee School of Dentistry, Memphis. He received the degree of Doctor of Dental Surgery in June, 1998, and the following month he entered his father's dental practice in Pine Mountain, Georgia. He worked with his father for six years and returned to The University of Tennessee School of Dentistry, Memphis, as a graduate student in Orthodontics and will receive his Master of Dental Science degree in Orthodontics in May, 2007.

He plans to join a group in private practice specializing in orthodontics in Collierville, Tennessee and Southaven, Mississippi. 